\title{
EXISTENCE OF LARGE-DATA GLOBAL-IN-TIME FINITE-ENERGY WEAK SOLUTIONS TO A COMPRESSIBLE FENE-P MODEL
}

\author{
JOHN W. BARRETT AND ENDRE SÜLI
}

\begin{abstract}
A compressible FENE-P type model with stress diffusion is derived from an approximate macroscopic closure of a compressible Navier-Stokes-Fokker-Planck system arising in the kinetic theory of dilute polymeric fluids, where polymer chains immersed in a barotropic, compressible, isothermal, viscous Newtonian solvent, are idealised as pairs of massless beads connected with finitely extensible nonlinear elastic (FENE) springs. We develop a priori bounds for the model, including logarithmic bounds, which guarantee the nonnegativity of the conformation tensor and a bound on its trace, and we prove the existence of large-data global-in-time finite-energy weak solutions in two and three space dimensions.
\end{abstract}

\section{INTRODUCTION}

In this paper we prove the existence of global-in-time weak solutions to the following compressible FENE-P model, posed in the time-space cylinder $(0, T] \times \Omega$, where $\Omega$ is a bounded open domain in $\mathbb{R}^{d}$ for $d=2$ or 3 :

$$
\begin{aligned}
\partial_{t} \varrho+\operatorname{div}_{x}(\varrho \mathbf{u}) & =0, \\
\partial_{t}(\varrho \mathbf{u})+\operatorname{div}_{x}(\varrho \mathbf{u} \otimes \mathbf{u})+\nabla_{x} p(\varrho)-\operatorname{div}_{x} \mathbb{S}\left(\nabla_{x} \mathbf{u}\right) & =\operatorname{div}_{x}(\mathbb{A}(\mathbb{T})-\Pi(\mathbb{T}) \mathbb{I})+\varrho \mathbf{f}, \\
\partial_{t} \mathbb{T}+\left(\mathbf{u} \cdot \nabla_{x}\right) \mathbb{T}-\left(\nabla_{x} \mathbf{u} \mathbb{T}+\mathbb{T} \nabla_{x}^{\mathrm{T}} \mathbf{u}\right) & =\varepsilon \Delta_{x} \mathbb{T}-\frac{1}{2 \lambda} \mathbb{A}(\mathbb{T}),
\end{aligned}
$$

where the pressure $p$ and the density $\varrho$ of the solvent are supposed to be related by the typical power law relation:

$$
p(\varrho)=a \varrho^{\gamma}, \quad a>0, \gamma>\frac{d}{2},
$$

and the Newtonian stress tensor $\mathbb{S}\left(\nabla_{x} \mathbf{u}\right)$ is defined by

$$
\mathbb{S}\left(\nabla_{x} \mathbf{u}\right)=\mu^{S}\left(\frac{\nabla_{x} \mathbf{u}+\nabla_{x}^{\mathrm{T}} \mathbf{u}}{2}-\frac{1}{d}\left(\operatorname{div}_{x} \mathbf{u}\right) \mathbb{I}\right)+\mu^{B}\left(\operatorname{div}_{x} \mathbf{u}\right) \mathbb{I},
$$

with constant shear and bulk viscosity coefficients, respectively, $\mu^{S}>0$ and $\mu^{B} \geq 0$. The velocity gradient matrix is defined as

$$
\left(\nabla_{x} \mathbf{u}\right)_{1 \leq i, j \leq d}=\left(\partial_{x_{j}} \mathbf{u}_{i}\right)_{1 \leq i, j \leq d}
$$

and $\mathbb{I} \in \mathbb{R}^{d \times d}$ is the identity matrix. The symmetric matrix function $\mathbb{T}=\left(\mathbb{T}_{i, j}\right), 1 \leq i, j \leq d$, defined on $[0, T] \times \Omega$, is the conformation tensor and $\operatorname{tr}(\mathbb{T})$ denotes its trace. The tensor $\mathbb{A}(\mathbb{T})$ is the extra stress tensor and is given by

$$
\mathbb{A}(\mathbb{T})=\left(1-\frac{\operatorname{tr}(\mathbb{T})}{\mathfrak{b}}\right)^{-1} \mathbb{T}-\mathfrak{a} \mathbb{I} .
$$

The scalar function $\Pi(\mathbb{T})$ is the polymeric pressure and is given by

$$
\Pi(\mathbb{T})=\frac{1}{2}\left[\mathfrak{a} \operatorname{tr}(\log (\mathbb{T}))+\mathfrak{b} \log \left(1-\frac{\operatorname{tr}(\mathbb{T})}{\mathfrak{b}}\right)+\mathfrak{b}\right],
$$

up to a constant, in order for the above system to satisfy an energy equality; see Section 3 for details. The constant here has been chosen for the convenience of later developments. The meaning of the parameters appearing in (1.1)-(1.3) will be introduced in the derivation of the model in Section 2. In particular, the parameters $\varepsilon, \mathfrak{a}, \mathfrak{b}$, and $\lambda$ are all positive constants. Of course, it is implicitly assumed, and has to be established, that, subject to a positive definite initial datum $\mathbb{T}_{0}$ whose trace is less than $\mathfrak{b}$, the tensor $\mathbb{T}$ remains positive definite with trace less than $\mathfrak{b}$ for all $t \in(0, T]$, so as to ensure that (1.7) and (1.8) are well-defined. In addition, the external force $\mathbf{f}$ is assumed to be an element of the

2010 Mathematics Subject Classification. 35A01, 35Q35, 76A05.

Key words and phrases. Weak solution; Compressible Navier-Stokes equation; FENE-P model. 
function space $L^{\infty}\left((0, T] \times \Omega ; \mathbb{R}^{d}\right)$. Finally, the equations (1.1)-(1.3) are supplemented by initial conditions for $\varrho$, $\mathbf{u}$ and $\mathbb{T}$, and the following boundary conditions for $\mathbf{u}$ and $\mathbb{T}$ :

$$
\begin{aligned}
\mathbf{u} & =\mathbf{0} & & \text { on }(0, T] \times \partial \Omega, \\
\left(\nu \cdot \nabla_{x}\right) \mathbb{T} & =\mathbb{O} & & \text { on }(0, T] \times \partial \Omega .
\end{aligned}
$$

Here $\nu$ is the outer unit normal vector on the boundary $\partial \Omega$ and $\mathbb{O} \in \mathbb{R}^{d \times d}$ is the zero matrix.

In contrast with the compressible FENE-P model considered here, in the case of the incompressible FENE-P model the pressure $p$ no longer satisfies (1.4) and acts as a Lagrange multiplier for the pointwise constraint $\operatorname{div}_{x} \mathbf{u}=0$. Moreover, the term $\nabla_{x} \Pi$ appearing on the right-hand side of (1.2) is absorbed into the pressure term $\nabla_{x} p$ on the left-hand side of the equation. For data $\mathbf{f} \equiv \mathbf{0}$ and initial data $\mathbf{u}_{0}$ and $\mathbb{T}_{0}$ for $\mathbf{u}$ and $\mathbb{T}$ such that $\mathbf{u}_{0} \in L^{2}\left(\Omega ; \mathbb{R}^{d}\right)$ is divergence free, and $\mathbb{T}_{0}$, which is symmetric positive definite for a.e. $x \in \Omega$, satisfying $\log \left(1-\frac{\operatorname{tr}\left(\mathbb{T}_{0}\right)}{\mathfrak{b}}\right) \in L^{1}\left(\Omega ; \mathbb{R}^{d \times d}\right)$, the existence of a global-in-time weak solution $\mathbf{u} \in L^{\infty}\left(0, T ; L^{2}\left(\Omega ; \mathbb{R}^{d}\right)\right) \cap L^{2}\left(0, T, H_{0}^{1}\left(\Omega ; \mathbb{R}^{d}\right)\right), \mathbb{T} \in L^{\infty}\left(0, T ; L^{\infty}\left(\Omega ; \mathbb{R}^{d \times d}\right)\right)$ and $\mathbb{A}(\mathbb{T}) \in L^{2}\left(0, T ; L^{2}\left(\Omega ; \mathbb{R}^{d \times d}\right)\right)$ was proved in Masmoudi [21] for the incompressible FENE-P model with constant density and in the absence of stress diffusion, i.e., $\varepsilon=0$, for $d=2$ and 3 . Convergence of a finite element approximation of such an incompressible FENE-P model with stress diffusion, $\varepsilon>0$, and $\mathbf{f} \in L^{2}\left(0, T ;\left[H_{0}^{1}\left(\Omega ; \mathbb{R}^{d}\right)\right]^{\prime}\right)$ was proved for $d=2$ in Barrett and Boyaval [2] under the slightly stronger assumptions on $\mathbb{T}_{0}: \mathbb{T}_{0}=\mathbb{T}_{0}^{\mathrm{T}} \geq \tau_{\min } \mathbb{I}$ and $\operatorname{tr}\left(\mathbb{T}_{0}\right) \leq \mathfrak{b}^{\star}<\mathfrak{b}$ a.e. in $\Omega$ with $\tau_{\min }>0$. Here $\mathbb{T}_{0} \geq \tau_{\min } \mathbb{I}$ means that $\mathbb{T}_{0}-\tau_{\min } \mathbb{I}$ is nonnegative definite.

\section{Derivation of the compressible FENE-P model}

In this section we introduce a compressible Navier-Stokes-Fokker-Planck system, which is slightly different from the model considered in Barrett and Süli $[4,5]$. We shall then (formally) derive from it the compressible FENE-P model (1.1)-(1.10) by considering the special case of the compressible FENE dumbbell model and formulating its approximate macroscopic closure.

2.1. Compressible Navier-Stokes-Fokker-Planck system. The solvent density $\varrho$ and the solvent velocity field $\mathbf{u}$ are defined on $[0, T] \times \Omega$ and $[0, T] \times \bar{\Omega}$, respectively, with $T>0$, and satisfy the compressible Navier-Stokes equations with an elastic extra stress-tensor $\mathbb{K}$ :

$$
\begin{aligned}
\partial_{t} \varrho+\operatorname{div}_{x}(\varrho \mathbf{u}) & =0 & & \text { in }(0, T] \times \Omega, \\
\partial_{t}(\varrho \mathbf{u})+\operatorname{div}_{x}(\varrho \mathbf{u} \otimes \mathbf{u})+\nabla_{x} p(\varrho)-\operatorname{div}_{x} \mathbb{S}\left(\nabla_{x} \mathbf{u}\right) & =\operatorname{div}_{x} \mathbb{K}+\varrho \mathbf{f} & & \text { in }(0, T] \times \Omega .
\end{aligned}
$$

The pressure $p(\varrho)$ and the Newtonian shear stress tensor $\mathbb{S}$ are defined by (1.4) and (1.5). We shall impose a no-slip boundary condition on the velocity field; i.e.,

$$
\mathbf{u}=\mathbf{0} \quad \text { on }(0, T] \times \partial \Omega .
$$

In the dumbbell model consisting of two beads coupled with an elastic spring representing a polymer chain, the nonNewtonian elastic extra stress tensor $\mathbb{K}$ is based on a version of the Kramers expression (cf. (2.7) below), depending on the probability density function $\psi$, which, in addition to $t$ and $x$, also depends on the conformation vector $q \in \mathbb{R}^{d}$ of the spring. Let $D \subset \mathbb{R}^{d}$ be the domain of admissible conformation vectors. Typically $D$ is the whole space $\mathbb{R}^{d}$ or a bounded open ball centered at the origin 0 in $\mathbb{R}^{d}$. Here we consider the FENE (finitely extensible nonlinear elastic) bead-spring model, where $D$ is the ball of radius $b^{\frac{1}{2}}$ centred at the origin, $B\left(0, b^{\frac{1}{2}}\right)$, with $b>2$. The corresponding elastic spring-force is $F: q \in D \mapsto U^{\prime}\left(\frac{1}{2}|q|^{2}\right) q \in \mathbb{R}^{d}$ with the spring potential $U:\left[0, \frac{b}{2}\right) \rightarrow \mathbb{R}_{\geq 0}$ defined by

$$
U(s)=-\frac{b}{2} \log \left(1-\frac{2 s}{b}\right), \quad \text { and so yielding } \quad F(q)=U^{\prime}\left(\frac{1}{2}|q|^{2}\right) q=\left(1-\frac{|q|^{2}}{b}\right)^{-1} q .
$$

The FENE model, as it only allows the springs to have finite extensions, is physically more realistic than the Hookean model, where $D=\mathbb{R}^{d}$ with $U:[0, \infty) \rightarrow \mathbb{R}_{\geq 0}$ defined by

$$
U(s)=s, \quad \text { and so yielding } \quad F(q)=U^{\prime}\left(\frac{1}{2}|q|^{2}\right) q=q .
$$

The extra stress tensor $\mathbb{K}$ in (2.2) is defined by the formula:

$$
\mathbb{K}(t, x):=k \mathbb{C}(\psi)(t, x)-\pi(t, x) \mathbb{I},
$$

where $k>0$ is the product of the Boltzmann constant and the absolute temperature, $\mathbb{C}$ is given by a form of Kramer's expression

$$
\mathbb{C}(\psi)(t, x):=\frac{\int_{D} \psi(t, x, q) U^{\prime}\left(\frac{|q|^{2}}{2}\right) q q^{\mathrm{T}} \mathrm{d} q}{\int_{D} \psi(t, x, q) \mathrm{d} q}
$$


and $\pi$ is a polymeric pressure term. In addition, we introduce the polymer number density

$$
\eta(t, x):=\int_{D} \psi(t, x, q) \mathrm{d} q .
$$

Since $\psi$ is a probability density function, it is nonnegative a.e. on $[0, T] \times \Omega \times D$ and satisfies the following FokkerPlanck equation:

$$
\partial_{t} \psi+\operatorname{div}_{x}(\mathbf{u} \psi)-\varepsilon \eta \Delta_{x}\left(\frac{\psi}{\eta}\right)=\operatorname{div}_{q}\left(\frac{1}{4 \lambda}\left(\nabla_{q} \psi+\psi F(q)\right)-\left(\nabla_{x} \mathbf{u}\right) q \psi\right) \quad \text { in }(0, T] \times \Omega \times D
$$

supplemented with the initial condition $\psi(0, x, q)=\psi_{0}(x, q) \geq 0$ for all $(x, q) \in \Omega \times D$, and the boundary conditions

$$
\begin{aligned}
{\left[\frac{1}{4 \lambda}\left(\nabla_{q} \psi+\psi F(q)\right)-\left(\nabla_{x} \mathbf{u}\right) q \psi\right] \cdot q=0 } & \text { on }(0, T] \times \Omega \times \partial D, \\
\varepsilon \eta \nabla_{x}\left(\frac{\psi}{\eta}\right) \cdot \nu=0 & \text { on }(0, T] \times \partial \Omega \times D,
\end{aligned}
$$

where $q$ is normal to $\partial D$. The constant parameters $\varepsilon>0$ and $\lambda>0$ are the centre-of-mass diffusion coefficient and the Deborah number, respectively; the latter characterises the elastic relaxation property of the solvent. The centre-of-mass diffusion term $\varepsilon \eta \Delta_{x}\left(\frac{\psi}{\eta}\right)$ in (2.9) and the corresponding boundary term $\varepsilon \eta \nabla_{x}\left(\frac{\psi}{\eta}\right)$ in (2.11) collapse to their standard forms $\varepsilon \Delta_{x} \psi$ and $\varepsilon \nabla_{x} \psi$, respectively, if $\eta$ is independent of $x$.

Integrating the partial differential equation (2.9) and its initial condition over $D$, using the boundary condition in (2.10) and noting (2.8), we deduce the following partial differential equation for the function $\eta$ :

$$
\partial_{t} \eta+\operatorname{div}_{x}(\mathbf{u} \eta)=0 \quad \text { on }(0, T] \times \Omega
$$

subject to the initial condition $\eta_{0}(x)=\int_{D} \psi_{0}(x, q) \mathrm{d} q$ for all $x \in \Omega$. Integrating (2.12) over $\Omega$ and noting (2.3) and (2.8) yields for a.e. $t \in[0, T]$ that

$$
\int_{\Omega \times D} \psi(t, x, q) \mathrm{d} x \mathrm{~d} q=\int_{\Omega} \eta(t, x) \mathrm{d} x=\int_{\Omega} \eta_{0}(x) \mathrm{d} x=\int_{\Omega \times D} \psi_{0}(x, q) \mathrm{d} x \mathrm{~d} q=1 .
$$

We note that in the incompressible case, with $\eta_{0} \equiv 1 /|\Omega|$, it follows from $(2.12)$ that $\eta(t, x)=\eta_{0}$ for all $(t, x) \in[0, T] \times \Omega$. Hence, as noted above, the centre-of-mass diffusion term in (2.9) and the corresponding boundary term in (2.11) collapse to their standard forms. In addition, on inserting (2.7) into (2.6) and noting (2.8) the extra stress tensor $\mathbb{K}$ collapses to a standard form of Kramer's rule

$$
\mathbb{K}(t, x)=k|\Omega| \int_{D} \psi(t, x, q) U^{\prime}\left(\frac{|q|^{2}}{2}\right) q q^{\mathrm{T}} \mathrm{d} q-\pi(t, x) .
$$

However, in the compressible case $\eta(t, x)$ is not constant; hence the need for the denominator in the definition of $\mathbb{C}$ as stated in (2.7).

Introducing

$$
\psi^{\star}(t, x, q)=\frac{\psi(t, x, q)}{|\Omega| \eta(t, x)} \quad \text { for all }(t, x, q) \in[0, T] \times \Omega \times D,
$$

it follows from (2.8), (2.9) and (2.12) that $\psi^{\star}(t, x, q) \geq 0, \int_{D} \psi^{\star}(t, x, q) \mathrm{d} q=1 /|\Omega|$ and satisfies

$$
\partial_{t} \psi^{\star}+\mathbf{u} \cdot \nabla_{x} \psi^{\star}-\varepsilon \Delta_{x} \psi^{\star}=\operatorname{div}_{q}\left(\frac{1}{4 \lambda}\left(\nabla_{q} \psi^{\star}+\psi^{\star} F(q)\right)-\left(\nabla_{x} \mathbf{u}\right) q \psi^{\star}\right) \quad \text { in }(0, T] \times \Omega \times D,
$$

supplemented with the initial condition $\psi^{\star}(0, x, q)=\frac{\psi_{0}(x, q)}{|\Omega| \eta_{0}(x)}$ for all $(x, q) \in \Omega \times D$, and the boundary conditions

$$
\begin{array}{rr}
{\left[\frac{1}{4 \lambda}\left(\nabla_{q} \psi^{\star}+\psi^{\star} F(q)\right)-\left(\nabla_{x} \mathbf{u}\right) q \psi^{\star}\right] \cdot q=0} & \text { on }(0, T] \times \Omega \times \partial D, \\
\varepsilon \nabla_{x} \psi^{\star} \cdot \nu=0 & \text { on }(0, T] \times \partial \Omega \times D .
\end{array}
$$

Moreover, we obtain from $(2.6),(2.7),(2.15)$ and $(2.8)$ that

$$
\mathbb{K}(t, x):=k|\Omega| \int_{D} \psi^{\star}(t, x, q) U^{\prime}\left(\frac{|q|^{2}}{2}\right) q q^{\mathrm{T}} \mathrm{d} q-\pi(t, x) \mathbb{I} .
$$

Finally, we note that in the incompressible case, with $\eta_{0} \equiv 1 /|\Omega|$, one has that $\psi^{\star}=\psi$.

Let $\mathcal{F}(s)=s(\log s-1)+1$ for $s \geq 0$, and suppose that $M$ is the Maxwellian associated with the potential $U$, (2.4); that is, $M(q)=C \mathrm{e}^{-U\left(\frac{1}{2}|q|^{2}\right)}$, where $C \in \mathbb{R}_{>0}$ is chosen so that $\int_{D} M \mathrm{~d} q=1$. Then the system (2.1)-(2.3), (2.16)-(2.19) supplemented with suitable initial conditions for $\varrho, \mathbf{u}, \psi^{\star}$ and the choice $\pi(t, x)=-k|\Omega| \int_{D} M(q) \mathcal{F}\left(\frac{\psi^{\star}(t, x, q)}{M(q)}\right) \mathrm{d} q$ in (2.6), in order for the above system to satisfy an energy equality, leads to a compressible Navier-Stokes-Fokker-Planck 
model in the case of FENE bead-spring chains, (2.4). Moreover, $-\pi$ is the part of the energy that is dependent on $\psi^{\star}$. Similarly, it turns out that $-\Pi(\mathbb{T})$ is the part of the energy that is dependent on $\mathbb{T}$ in the energy equality for (1.1)-(1.10); see (3.23) below. The above FENE model is slightly different from the one considered in $[4,5]$. There we did not include the denominator in the definition of $\mathbb{C},(2.7)$, leading to $(2.14)$ for $\mathbb{K}$. In addition, we did not include the $\eta$ factors in (2.9) and (2.11). We proved existence of a global-in-time weak solution to the system (2.1)-(2.4), (2.14) with (2.9)-(2.11) independent of $\eta$, supplemented with suitable initial conditions for $\varrho, \mathbf{u}, \psi$ and the choice $\pi(t, x)=L \eta(t, x)+\mathfrak{z} \eta^{2}(t, x)$ in (2.14), in order for the above system to satisfy an energy equality, with $L>0$ and $\mathfrak{z} \geq 0$ for $d=2$ in [5] and $L \geq 0$ and $\mathfrak{z}>0$ for $d=3$ in [4]. Note that, with (2.9)-(2.11) independent of $\eta$, one has that the function $\eta$, defined by (2.8), appearing in $\pi$, satisfies the following modification of (2.12):

$$
\partial_{t} \eta+\operatorname{div}_{x}(\mathbf{u} \eta)-\varepsilon \Delta_{x} \eta=0 \quad \text { on }(0, T] \times \Omega \quad \text { and } \quad \varepsilon \nabla_{x} \eta \cdot \nu=0 \quad \text { on }(0, T] \times \partial \Omega
$$

subject to the initial condition $\eta_{0}(x)=\int_{D} \psi_{0}(x, q) \mathrm{d} q$ for all $x \in \Omega$. It is a simple matter to adapt the proofs in those papers to prove existence of a global-in-time weak solution to the system (2.1)-(2.4), (2.16)-(2.19) supplemented with suitable initial conditions for $\varrho, \mathbf{u}, \psi^{\star}$ and the choice $\pi(t, x)=-k|\Omega| \int_{D} M(q) \mathcal{F}\left(\frac{\psi^{\star}(t, x, q)}{M(q)}\right) \mathrm{d} q$ in $(2.19)$ for $d=2$ or 3 . In fact, the proofs in the above papers are for FENE chains consisting of $K+1$ beads and $K$ springs. The dumbell case considered here corresponds to the special case of $K=1$.

In the next section we use formal computations to derive an approximate closure of the above compressible NavierStokes-Fokker-Planck FENE model, which leads to the compressible FENE-P model (1.1)-(1.10), whose analysis is thereafter pursued in the rest of the paper.

2.2. Compressible FENE-P model. This section is devoted to the derivation of the model (1.1)-(1.10) from the Navier-Stokes-Fokker-Planck FENE system stated in Section 2.1, consisting of the equations (2.1), (2.2), (2.16), the definitions (2.19), (2.4), the boundary conditions (2.3), (2.17), (2.18), suitable initial conditions for $\varrho, \mathbf{u}, \psi^{\star}$ and a suitable choice for $\pi$ in (2.19).

We introduce the symmetric tensor

$$
\mathbb{T}(t, x):=k|\Omega| \int_{D} \psi^{\star}(t, x, q) q q^{\mathrm{T}} \mathrm{d} q, \quad \text { and so } \quad \operatorname{tr}(\mathbb{T})(t, x)=k|\Omega| \int_{D} \psi^{\star}(t, x, q)|q|^{2} \mathrm{~d} q
$$

By noting (2.19), (2.4) and that $\int_{D} \psi^{\star} \mathrm{d} q=1 /|\Omega|$, we then apply a Peterlin type approximation, see $[23,6,18]$, to $\mathbb{K}$ and obtain

$$
\begin{aligned}
\mathbb{K}(t, x) & =k|\Omega| \int_{D} \psi^{\star}(t, x, q)\left(1-\frac{|q|^{2}}{b}\right)^{-1} q q^{\mathrm{T}} \mathrm{d} q-\pi(t, x) \mathbb{I} \\
& \approx k|\Omega| \int_{D} \psi^{\star}(t, x, q)\left(1-\frac{|\Omega| \int_{D} \psi^{\star}(t, x, q)|q|^{2} \mathrm{~d} q}{b}\right)^{-1} q q^{\mathrm{T}} \mathrm{d} q-\pi(t, x) \mathbb{I} \\
& =\left(1-\frac{\operatorname{tr}(\mathbb{T})}{\mathfrak{b}}\right)^{-1} \mathbb{T}-\pi(t, x) \mathbb{I}
\end{aligned}
$$

where $\mathfrak{b}=k b$.

The continuity equation (1.1) for the solvent density $\varrho$ follows directly from (2.1). From (2.2), (2.22) and (1.7) with $\pi(t, x)=\Pi(\mathbb{T}(t, x))$, we deduce the balance of momentum equation (1.2); the boundary condition for the velocity field $\mathbf{u},(1.9)$, follows from (2.3).

The boundary condition (1.10) for $\mathbb{T}$ can be deduced from (2.18) and (2.21). It remains to derive the evolution equation (1.3) for the conformation tensor $\mathbb{T}$. To this end, we note the definition of $\mathbb{T}$ in (2.21), multiply equation (2.16) by the matrix $k|\Omega| q q^{\mathrm{T}}$, and integrate it with respect to $q$ over $D$. The left-hand side of (2.16) yields that

$$
k|\Omega| \int_{D}\left(\partial_{t} \psi^{\star}+\mathbf{u} \cdot \nabla_{x} \psi^{\star}-\varepsilon \Delta_{x} \psi^{\star}\right) q q^{\mathrm{T}} \mathrm{d} q=\partial_{t} \mathbb{T}+\mathbf{u} \cdot \nabla_{x} \mathbb{T}-\varepsilon \Delta_{x} \mathbb{T} .
$$


The right-hand side of (2.16) yields, on integrating by parts and noting the boundary condition (2.10) and (2.4), that

$$
\begin{aligned}
& k|\Omega| \int_{D} \operatorname{div}_{q}\left(\frac{1}{4 \lambda}\left(\nabla_{q} \psi^{\star}+\psi^{\star} F(q)\right)-\left(\nabla_{x} \mathbf{u}\right) q \psi^{\star}\right) q q^{\mathrm{T}} \mathrm{d} q \\
& =-k|\Omega| \int_{D}\left(\left(\frac{1}{4 \lambda}\left(\nabla_{q} \psi^{\star}+\psi^{\star} F(q)\right)-\left(\nabla_{x} \mathbf{u}\right) q \psi^{\star}\right) \cdot \nabla_{q}\right)\left(q q^{\mathrm{T}}\right) \mathrm{d} q \\
& =-\frac{k|\Omega|}{4 \lambda} \int_{D}\left(\left(\nabla_{q} \psi^{\star}\right) q^{\mathrm{T}}+q\left(\nabla_{q} \psi^{\star}\right)^{\mathrm{T}}\right) \mathrm{d} q-\frac{k|\Omega|}{2 \lambda} \int_{D} \psi^{\star}\left(1-\frac{|q|^{2}}{b}\right)^{-1} q q^{\mathrm{T}} \mathrm{d} q \\
& \quad+k|\Omega| \int_{D} \psi^{\star}\left(\left(\left(\nabla_{x} \mathbf{u}\right) q\right) q^{\mathrm{T}}+q\left(\left(\nabla_{x} \mathbf{u}\right) q\right)^{\mathrm{T}}\right) \mathrm{d} q=: \mathbb{B}_{1}+\mathbb{B}_{2}+\mathbb{B}_{3} .
\end{aligned}
$$

By recalling (2.21), we have that

$$
\mathbb{B}_{3}=\left(\nabla_{x} \mathbf{u}\right) \mathbb{T}+\mathbb{T}\left(\nabla_{x}^{\mathrm{T}} \mathbf{u}\right) .
$$

By performing the same approximation as used in (2.22) we deduce that

$$
\mathbb{B}_{2} \approx-\frac{1}{2 \lambda}\left(1-\frac{\operatorname{tr}(\mathbb{T})}{\mathfrak{b}}\right)^{-1} \mathbb{T}
$$

Performing integration by parts and ignoring the boundary term on $\partial D$ yields that

$$
\mathbb{B}_{1} \approx \frac{k}{2 \lambda} \mathbb{I}
$$

on recalling that $\int_{D} \psi^{\star} \mathrm{d} q=1 /|\Omega|$. By combining (2.16), (2.23)-(2.27) and recalling (1.7) we deduce the equation (1.3) for $\mathbb{T}$ with $\mathfrak{a}=k$ and $\mathfrak{b}=k b$. We note that if $\mathbb{T}$ solves (1.3) for a given $\mathbf{u}$, then taking the transpose of (1.3) we see that $\mathbb{T}^{\mathrm{T}}$ solves (1.3). Hence the symmetry of $\mathbb{T}$ from the definition (2.21) is encoded in the macroscopic equation (1.3). Finally, the choice of $\pi(t, x)=\Pi(\mathbb{T}(t, x))$ in (1.8) leads to (1.1)-(1.10) satisfying an energy equality; see Section 3 below, and in particular (3.20), for details. It should be noted that $-\Pi(\mathbb{T})$ is the part of the energy that is dependent on $\mathbb{T}$; see (3.23) below.

Formally, letting $\mathfrak{b} \rightarrow \infty$ in (1.2), (1.3) and (1.8) one obtains that

$$
\left(1-\frac{\operatorname{tr}(\mathbb{T})}{\mathfrak{b}}\right)^{-1} \rightarrow 1 \quad \text { and } \quad \mathfrak{b} \log \left(1-\frac{\operatorname{tr}(\mathbb{T})}{\mathfrak{b}}\right) \rightarrow-\operatorname{tr}(\mathbb{T})
$$

This gives rise to a compressible Oldroyd-B model, which is slightly different from the one analysed in Barrett, Lu and Süli [3]. In that paper we considered the formal macroscopic closure of the compressible Hookean system (2.1)-(2.3), (2.14) and (2.9)-(2.11) independent of $\eta$, with (2.5) in place of (2.4). This leads to the $\mathfrak{b} \rightarrow \infty$ limit of (1.1)-(1.10) as above, but with the following modifications: (i) $\mathfrak{a}$ in (1.3) is replaced with $\mathfrak{a} \eta$, and (ii) (1.8) is replaced with $\Pi(\eta)=L \eta+\mathfrak{z} \eta^{2}$ for $L, \mathfrak{z} \geq 0$, where $\eta$ satisfies (2.20). In [3] we proved existence of a global-in-time weak solution in two space dimensions $(d=2)$, with at least one of $L$ and $\mathfrak{z}$ assumed to be nonzero. The proof there is easily adapted to the compressible Oldroyd-B model, which is the direct $\mathfrak{b} \rightarrow \infty$ limit of (1.1)-(1.10).

Having derived our compressible FENE-P model, (1.1)-(1.10), we now focus our attention on its mathematical analysis. We begin by establishing a priori bounds that will form the basis of the weak compactness argument leading to the proof of existence of a global-in-time weak solution to the system under consideration.

\section{A PRIORI BOUNDS}

This section is devoted to the derivation of formal a priori bounds for the compressible FENE-P model (1.1)-(1.8) subject to the boundary conditions (1.9), (1.10), and given initial conditions.

3.1. Initial data and a priori bound. We adopt the following hypotheses on the initial data:

$$
\begin{aligned}
& \varrho(0, \cdot)=\varrho_{0}(\cdot) \text { with } \varrho_{0} \geq 0 \text { a.e. in } \Omega, \quad \varrho_{0} \in L^{\gamma}(\Omega), \\
& \mathbf{u}(0, \cdot)=\mathbf{u}_{0}(\cdot) \in L^{r}\left(\Omega ; \mathbb{R}^{d}\right) \text { for some } r \geq 2 \gamma^{\prime} \text { such that } \varrho_{0}\left|\mathbf{u}_{0}\right|^{2} \in L^{1}(\Omega), \\
& \mathbb{T}(0, \cdot)=\mathbb{T}_{0}(\cdot) \text { with } \mathbb{T}_{0}=\mathbb{T}_{0}^{\mathrm{T}} \geq \tau_{\min } \mathbb{I} \text { and } \operatorname{tr}\left(\mathbb{T}_{0}\right) \leq \mathfrak{b}^{\star}<\mathfrak{b} \text { a.e. in } \Omega, \text { where } \tau_{\min } \in\left(0, \frac{\mathfrak{b}^{\star}}{d}\right] .
\end{aligned}
$$

Here $\gamma^{\prime}$ denotes the conjugate exponent to $\gamma>\frac{d}{2}$ for $d \in\{2,3\}$, i.e., $1 / \gamma+1 / \gamma^{\prime}=1$.

Because the density $\varrho$ is required to be a nonnegative function, we have assumed that the initial datum $\varrho_{0}$ is nonnegative. Since the scaled probability density function $\psi^{\star},(2.15)$, is nonnegative, then the definition of $\mathbb{T}$ stated in (2.21) from the Navier-Stokes-Fokker-Planck system, (2.1)-(2.4), (2.16)-(2.19), automatically implies that $\mathbb{T}$ must be a symmetric nonnegative definite matrix a.e. on $(0, T] \times \Omega$. However, this information on $\mathbb{T}$ is not a priori encoded in the macroscopic counterpart of this kinetic model, the compressible FENE-P model (1.1)-(1.10). Furthermore, because 
of the right-hand sides of $(1.2),(1.3)$ and $\Pi(\mathbb{T})$, see $(1.8)$, we require $\mathbb{T}>0$, i.e., $\mathbb{T}$ is positive definite, and tr $(\mathbb{T})<\mathfrak{b}$ a.e. in $(0, T] \times \Omega$. We have assumed slightly stronger constraints on the initial datum for $\mathbb{T}$ in $(3.1)$; see Remark 6.1 below . For the purposes of the formal energy estimates developed in this section, we will temporarily assume that ( $\varrho, \mathbf{u}, \mathbb{T}$ ) is a smooth solution to (1.1)-(1.10), (3.1) with $\varrho \geq 0, \mathbb{T}>0$ and $\operatorname{tr}(\mathbb{T})<\mathfrak{b}$ in $[0, T] \times \bar{\Omega}$. We stress that the energy estimates below, and these constraints on $\varrho$ and $\mathbb{T}$, will be made rigorous later in the paper.

We deduce from $(3.1)_{1}$ and $(3.1)_{2}$ by using Hölder's inequality that

$$
(\varrho \mathbf{u})(0, \cdot)=\varrho_{0} \mathbf{u}_{0}=\sqrt{\varrho_{0}} \sqrt{\varrho_{0}} \mathbf{u}_{0} \in L^{\frac{2 \gamma}{\gamma+1}}\left(\Omega ; \mathbb{R}^{d}\right) .
$$

For the solvent density $\varrho$, integration of (1.1) over $\Omega$ with respect to the spatial variable $x$, performing partial integration and noting the no-slip boundary condition (1.9) for the velocity field gives

$$
\frac{\mathrm{d}}{\mathrm{d} t} \int_{\Omega} \varrho(t, x) \mathrm{d} x=0 \Longrightarrow \int_{\Omega} \varrho(t, x) \mathrm{d} x=\int_{\Omega} \varrho_{0}(x) \mathrm{d} x, \quad t \in(0, T]
$$

In order to derive a formal energy identity we then take the inner product of the momentum equation (1.2) with the velocity field $\mathbf{u}$, integrate over $\Omega$ with respect to the spatial variable $x$, and perform partial integration noting the no-slip boundary condition (1.9) for $\mathbf{u}$. In order to explain the details of the calculation, we shall perform the computations term by term. We begin by noting that for the first term in (1.2) we have

$$
\int_{\Omega} \partial_{t}(\varrho \mathbf{u}) \cdot \mathbf{u} \mathrm{d} x=\int_{\Omega}\left(\partial_{t} \varrho\right)|\mathbf{u}|^{2} \mathrm{~d} x+\int_{\Omega} \varrho \partial_{t} \frac{|\mathbf{u}|^{2}}{2} \mathrm{~d} x=\frac{1}{2} \frac{\mathrm{d}}{\mathrm{d} t} \int_{\Omega} \varrho|\mathbf{u}|^{2} \mathrm{~d} x+\frac{1}{2} \int_{\Omega}\left(\partial_{t} \varrho\right)|\mathbf{u}|^{2} \mathrm{~d} x, \quad t \in(0, T] .
$$

For the second term in $(1.2)$,

$$
\begin{aligned}
\int_{\Omega} \operatorname{div}_{x}(\varrho \mathbf{u} \otimes \mathbf{u}) \cdot \mathbf{u} \mathrm{d} x & =-\int_{\Omega}(\varrho \mathbf{u} \otimes \mathbf{u}): \nabla_{x} \mathbf{u} \mathrm{d} x=-\sum_{i, j=1}^{d} \int_{\Omega} \varrho \mathbf{u}_{i} \mathbf{u}_{j} \partial_{x_{j}} \mathbf{u}_{i} \mathrm{~d} x \\
& =-\sum_{i, j=1}^{d} \int_{\Omega} \varrho \mathbf{u}_{j} \frac{1}{2} \partial_{x_{j}}\left|\mathbf{u}_{i}\right|^{2} \mathrm{~d} x=\frac{1}{2} \int_{\Omega} \operatorname{div}_{x}(\varrho \mathbf{u})|\mathbf{u}|^{2} \mathrm{~d} x, \quad t \in(0, T]
\end{aligned}
$$

By recalling (1.4), we have for the third term in (1.2) that

$$
\int_{\Omega} \nabla_{x} p(\varrho) \cdot \mathbf{u} \mathrm{d} x=-\int_{\Omega}\left(a \varrho^{\gamma}\right) \operatorname{div}_{x} \mathbf{u} \mathrm{d} x, \quad t \in(0, T] .
$$

Multiplication of (1.1) by $\gamma \varrho^{\gamma-1}$ gives

$$
\partial_{t} \varrho^{\gamma}+\operatorname{div}_{x}\left(\varrho^{\gamma} \mathbf{u}\right)+(\gamma-1) \varrho^{\gamma} \operatorname{div}_{x} \mathbf{u}=0 .
$$

Thus, thanks to the boundary condition (1.9) and our assumption that $\gamma>\frac{d}{2}$ with $d \in\{2,3\}$, we have that

$$
\int_{\Omega} \nabla_{x} p(\varrho) \cdot \mathbf{u} \mathrm{d} x=\int_{\Omega} \frac{a}{\gamma-1}\left(\partial_{t} \varrho^{\gamma}+\operatorname{div}_{x}\left(\varrho^{\gamma} \mathbf{u}\right)\right) \mathrm{d} x=\frac{a}{\gamma-1} \frac{\mathrm{d}}{\mathrm{d} t} \int_{\Omega} \varrho^{\gamma} \mathrm{d} x, \quad t \in(0, T] .
$$

For the fourth term in (1.2) we have

$$
\begin{aligned}
-\int_{\Omega} \operatorname{div}_{x} \mathbb{S}\left(\nabla_{x} \mathbf{u}\right) \cdot \mathbf{u} \mathrm{d} x & =\int_{\Omega}\left(\mu^{S}\left(\frac{\nabla_{x} \mathbf{u}+\nabla_{x}^{\mathrm{T}} \mathbf{u}}{2}-\frac{1}{d}\left(\operatorname{div}_{x} \mathbf{u}\right) \mathbb{I}\right)+\mu^{B}\left(\operatorname{div}_{x} \mathbf{u}\right) \mathbb{I}\right): \nabla_{x} \mathbf{u} \mathrm{d} x \\
& =\int_{\Omega}\left[\mu^{S}\left|\frac{\nabla_{x} \mathbf{u}+\nabla_{x}^{\mathrm{T}} \mathbf{u}}{2}-\frac{1}{d}\left(\operatorname{div}_{x} \mathbf{u}\right) \mathbb{I}\right|^{2}+\mu^{B}\left|\operatorname{div}_{x} \mathbf{u}\right|^{2}\right] \mathrm{d} x, \quad t \in(0, T]
\end{aligned}
$$

Recalling our present assumptions on $\mathbb{T}$, we note that (1.7) and (1.8) are well-defined. We then have for the fifth term in (1.2) that

$$
\int_{\Omega} \operatorname{div}_{x}(\mathbb{A}(\mathbb{T})-\Pi(\mathbb{T}) \mathbb{I}) \cdot \mathbf{u} \mathrm{d} x=-\int_{\Omega}(\mathbb{A}(\mathbb{T})-\Pi(\mathbb{T}) \mathbb{I}): \nabla_{x} \mathbf{u} \mathrm{d} x, \quad t \in(0, T] .
$$

Therefore, combining (1.2) and (3.2)-(3.6), we deduce, by noting (1.1), that

$$
\begin{gathered}
\frac{\mathrm{d}}{\mathrm{d} t} \int_{\Omega}\left[\frac{1}{2} \varrho|\mathbf{u}|^{2}+\frac{a}{\gamma-1} \varrho^{\gamma}\right] \mathrm{d} x+\int_{\Omega} \mu^{S}\left[\left|\frac{\nabla_{x} \mathbf{u}+\nabla_{x}^{\mathrm{T}} \mathbf{u}}{2}-\frac{1}{d}\left(\operatorname{div}_{x} \mathbf{u}\right) \mathbb{I}\right|^{2}+\mu^{B}\left|\operatorname{div}_{x} \mathbf{u}\right|^{2}\right] \mathrm{d} x \\
=-\int_{\Omega}(\mathbb{A}(\mathbb{T})-\Pi(\mathbb{T}) \mathbb{I}): \nabla_{x} \mathbf{u} \mathrm{d} x+\int_{\Omega} \varrho \mathbf{f} \cdot \mathbf{u} \mathrm{d} x, \quad t \in(0, T] .
\end{gathered}
$$

In order to complete the derivation of a (formal) energy identity for the system, we need to deal with the first term on the right-hand side of equation (3.7). Here, we recall the energy structure for the incompressible FENE-P system in the absence of stress diffusion, see Wapperom and Hulsen [26] and $\mathrm{Hu}$ and Lelièvre [17], and in the presence of stress 
diffusion, see Barrett and Boyaval [2]. Recalling our present assumptions on $\mathbb{T}$, we will take the inner product of the stress equation (1.3) with

$$
\left(1-\frac{\operatorname{tr}(\mathbb{T})}{\mathfrak{b}}\right)^{-1} \mathbb{I}-\mathfrak{a} \mathbb{T}^{-1}=\mathbb{A}(\mathbb{T}) \mathbb{T}^{-1}
$$

First, we recall the following formula, usually referred to as Jacobi's formula:

$$
\partial(\operatorname{det} \mathbb{T})=(\operatorname{det} \mathbb{T}) \operatorname{tr}\left(\mathbb{T}^{-1} \partial \mathbb{T}\right) ; \quad \text { hence } \quad \partial(\log \operatorname{det} \mathbb{T})=\operatorname{tr}\left(\mathbb{T}^{-1} \partial \mathbb{T}\right)=\partial \mathbb{T}: \mathbb{T}^{-1}
$$

where, in the present context, $\partial$ is a derivative in space or time. Since $\mathbb{T}$ is symmetric positive definite, we can define its real $\log$ arithm, $\log \mathbb{T}$, which is a symmetric matrix via diagonalisation of $\mathbb{T}$. Using the orthogonal $d \times d$ matrix $\mathbb{Q}$, we have that

$$
\mathbb{T}=\mathbb{Q} \operatorname{diag}\left\{\mathfrak{l}_{1}, \mathfrak{l}_{2}, \ldots, \mathfrak{l}_{d}\right\} \mathbb{Q}^{\mathrm{T}} \quad \text { and therefore } \quad \log \mathbb{T}=\mathbb{Q} \operatorname{diag}\left\{\log \mathfrak{l}_{1}, \log \mathfrak{l}_{2}, \ldots, \log \mathfrak{l}_{d}\right\} \mathbb{Q}^{\mathrm{T}},
$$

where $\mathfrak{l}_{\kappa}>0, \kappa=1, \ldots, d$, are the eigenvalues of $\mathbb{T}$. Since $\operatorname{tr}\left(\mathbb{B}_{1} \mathbb{B}_{2}\right)=\operatorname{tr}\left(\mathbb{B}_{2} \mathbb{B}_{1}\right)$ for all $\mathbb{B}_{1}, \mathbb{B}_{2} \in \mathbb{R}^{d \times d}$, we have the following identity:

$$
\operatorname{tr}(\log \mathbb{T})=\log \operatorname{det} \mathbb{T}
$$

By (3.9) and (3.11), we have that

$$
\partial \mathbb{T}: \mathbb{T}^{-1}=\partial(\log \operatorname{det} \mathbb{T})=\partial[\operatorname{tr}(\log \mathbb{T})]
$$

In addition, we note the following lemma.

Lemma 3.1. Let $\mathbb{P} \in W^{2,2}\left(\Omega ; \mathbb{R}^{m \times m}\right) \cap C^{1}\left(\bar{\Omega} ; \mathbb{R}^{m \times m}\right), m \in \mathbb{N}$, be a symmetric matrix function, which is positive definite, uniformly on $\bar{\Omega}$, and satisfies $\left(\nu \cdot \nabla_{x}\right) \mathbb{P}=\mathbb{O}$ on $\partial \Omega$; then,

$$
\int_{\Omega} \Delta_{x} \mathbb{P}: \mathbb{P}^{-1} \mathrm{~d} x=\sum_{j=1}^{d} \int_{\Omega} \operatorname{tr}\left(\left(\left(\partial_{x_{j}} \mathbb{P}\right) \mathbb{P}^{-1}\right)^{2}\right) \mathrm{d} x \geq \frac{1}{m} \int_{\Omega}\left|\nabla_{x} \operatorname{tr}(\log \mathbb{P})\right|^{2} \mathrm{~d} x .
$$

Proof. This lemma is stated as Lemma 3.1, and proved in Appendix A, of [3] in the case $m=d$. The proof given there immediately generalises to $m \in \mathbb{N}$.

Below we assume for this formal energy identity that $\mathbb{T}$ is sufficiently smooth to satisfy the assumptions of the above Lemma, as well as $\operatorname{tr}(\mathbb{T})<\mathfrak{b}$ a.e. in $(0, T] \times \Omega$. We now take the inner product of $(1.3)$ with $(3.8)$ integrate over $\Omega$ with respect to $x$, perform partial integration and note the boundary conditions $(1.9),(1.10)$. We have for the first term, by noting (3.12) and (1.8), that

$$
\begin{aligned}
\int_{\Omega} \partial_{t} \mathbb{T}:\left[\left(1-\frac{\operatorname{tr}(\mathbb{T})}{\mathfrak{b}}\right)^{-1} \mathbb{I}-\mathfrak{a}^{-1}\right] \mathrm{d} x & =-\frac{\mathrm{d}}{\mathrm{d} t} \int_{\Omega}\left[\mathfrak{a} \operatorname{tr}(\log \mathbb{T})+\mathfrak{b} \log \left(1-\frac{\operatorname{tr}(\mathbb{T})}{\mathfrak{b}}\right)\right] \mathrm{d} x \\
& =-2 \frac{\mathrm{d}}{\mathrm{d} t} \int_{\Omega} \Pi(\mathbb{T}) \mathrm{d} x, \quad t \in(0, T] .
\end{aligned}
$$

Here, and throughout, we recall that $\mathbb{B}_{1}: \mathbb{B}_{2}=\operatorname{tr}\left(\mathbb{B}_{1}^{\mathrm{T}} \mathbb{B}_{2}\right)=\operatorname{tr}\left(\mathbb{B}_{2}^{\mathrm{T}} \mathbb{B}_{2}\right)$ for all $\mathbb{B}_{1}, \mathbb{B}_{2} \in \mathbb{R}^{d \times d}$. Similarly, for the second term in (1.3) we have that

$$
\begin{aligned}
\int_{\Omega}\left(\mathbf{u} \cdot \nabla_{x}\right) \mathbb{T}:\left[\left(1-\frac{\operatorname{tr}(\mathbb{T})}{\mathfrak{b}}\right)^{-1} \mathbb{I}-\mathfrak{a} \mathbb{T}^{-1}\right] \mathrm{d} x & =-2 \int_{\Omega} \mathbf{u} \cdot \nabla_{x} \Pi(\mathbb{T}) \mathrm{d} x=2 \int_{\Omega} \Pi(\mathbb{T}) \operatorname{div}_{x} \mathbf{u} \mathrm{d} x \\
& =2 \int_{\Omega} \Pi(\mathbb{T}) \mathbb{I}: \nabla_{x} \mathbf{u} \mathrm{d} x, \quad t \in(0, T]
\end{aligned}
$$

For the third term in (1.3), we have, by recalling (1.7), that

$$
\begin{aligned}
-\int_{\Omega}\left(\nabla_{x} \mathbf{u} \mathbb{T}+\mathbb{T} \nabla_{x}^{\mathrm{T}} \mathbf{u}\right):\left[\left(1-\frac{\operatorname{tr}(\mathbb{T})}{\mathfrak{b}}\right)^{-1} \mathbb{I}-\mathfrak{a} \mathbb{T}^{-1}\right] \mathrm{d} x & =-2 \int_{\Omega}\left[\left(1-\frac{\operatorname{tr}(\mathbb{T})}{\mathfrak{b}}\right)^{-1} \operatorname{tr}\left(\mathbb{T} \nabla_{x} \mathbf{u}\right)-\mathfrak{a} \operatorname{div} \mathbf{u}_{x} \mathbf{\mathrm { d } x}\right. \\
& =-2 \int_{\Omega} \mathbb{A}(\mathbb{T}): \nabla_{x} \mathbf{u} \mathrm{d} x, \quad t \in(0, T]
\end{aligned}
$$


For the fourth term in (1.3), we have, by noting (3.13), that

$$
\begin{aligned}
\varepsilon \int_{\Omega} \Delta_{x} \mathbb{T}: & {\left[\left(1-\frac{\operatorname{tr}(\mathbb{T})}{\mathfrak{b}}\right)^{-1} \mathbb{I}-\mathfrak{a} \mathbb{T}^{-1}\right] \mathrm{d} x } \\
= & -\varepsilon \int_{\Omega}\left[\mathfrak{b} \Delta_{x}\left(1-\frac{\operatorname{tr}(\mathbb{T})}{\mathfrak{b}}\right)\left(1-\frac{\operatorname{tr}(\mathbb{T})}{\mathfrak{b}}\right)^{-1}+\mathfrak{a} \Delta_{x} \mathbb{T}: \mathbb{T}^{-1}\right] \mathrm{d} x \\
= & \left.-\varepsilon \sum_{j=1}^{d} \int_{\Omega}\left[\mathfrak{b}\left(\left(\partial_{x_{j}}\left(1-\frac{\operatorname{tr}(\mathbb{T})}{\mathfrak{b}}\right)\right)\left(1-\frac{\operatorname{tr}(\mathbb{T})}{\mathfrak{b}}\right)^{-1}\right)^{2}+\mathfrak{a} \operatorname{tr}\left(\left(\partial_{x_{j}} \mathbb{T}\right) \mathbb{T}^{-1}\right)^{2}\right)\right] \mathrm{d} x, \quad t \in(0, T] .
\end{aligned}
$$

For the fifth term in (1.3), we have, by recalling (1.7) and (3.8), that

$$
-\frac{1}{2 \lambda} \int_{\Omega} \mathbb{A}(\mathbb{T}):\left(\mathbb{A}(\mathbb{T}) \mathbb{T}^{-1}\right) \mathrm{d} x=-\frac{1}{2 \lambda} \int_{\Omega} \operatorname{tr}\left((\mathbb{A}(\mathbb{T}))^{2} \mathbb{T}^{-1}\right) \mathrm{d} x, \quad t \in(0, T] .
$$

Therefore, combining (1.3) and (3.14)-(3.18), we deduce

$$
\begin{aligned}
& -2 \frac{\mathrm{d}}{\mathrm{d} t} \int_{\Omega} \Pi(\mathbb{T}) \mathrm{d} x+\frac{1}{2 \lambda} \int_{\Omega} \operatorname{tr}\left((\mathbb{A}(\mathbb{T}))^{2} \mathbb{T}^{-1}\right) \mathrm{d} x \\
& \quad+\varepsilon \sum_{j=1}^{d} \int_{\Omega}\left[\mathfrak{b}\left(\left(\partial_{x_{j}}\left(1-\frac{\operatorname{tr}(\mathbb{T})}{\mathfrak{b}}\right)\right)\left(1-\frac{\operatorname{tr}(\mathbb{T})}{\mathfrak{b}}\right)^{-1}\right)^{2}+\mathfrak{a} \operatorname{tr}\left(\left(\left(\partial_{x_{j}} \mathbb{T}\right) \mathbb{T}^{-1}\right)^{2}\right)\right] \mathrm{d} x \\
& =2 \int_{\Omega}(\mathbb{A}(\mathbb{T})-\Pi(\mathbb{T}) \mathbb{I}): \nabla_{x} \mathbf{u} \mathrm{d} x, \quad t \in(0, T] .
\end{aligned}
$$

By adding $\frac{1}{2}(3.19)$ to the a priori bound (3.7), we finally obtain the following (formal) energy equality:

$$
\begin{aligned}
& \frac{\mathrm{d}}{\mathrm{d} t} \int_{\Omega}\left[\frac{1}{2} \varrho|\mathbf{u}|^{2}+\right.\left.\frac{a}{\gamma-1} \varrho^{\gamma}-\Pi(\mathbb{T})\right] \mathrm{d} x+\int_{\Omega}\left[\mu^{S}\left|\frac{\nabla_{x} \mathbf{u}+\nabla_{x}^{\mathrm{T}} \mathbf{u}}{2}-\frac{1}{d}\left(\operatorname{div}_{x} \mathbf{u}\right) \mathbb{I}\right|^{2}+\mu^{B}\left|\operatorname{div}_{x} \mathbf{u}\right|^{2}\right] \mathrm{d} x \\
&+\frac{1}{4 \lambda} \int_{\Omega} \operatorname{tr}\left((\mathbb{A}(\mathbb{T}))^{2} \mathbb{T}^{-1}\right) \mathrm{d} x \\
&+\frac{\varepsilon}{2} \sum_{j=1}^{d} \int_{\Omega}\left[\mathfrak{b}\left(\left(\partial_{x_{j}}\left(1-\frac{\operatorname{tr}(\mathbb{T})}{\mathfrak{b}}\right)\right)\left(1-\frac{\operatorname{tr}(\mathbb{T})}{\mathfrak{b}}\right)^{-1}\right)^{2}+\mathfrak{a} \operatorname{tr}\left(\left(\left(\partial_{x_{j}} \mathbb{T}\right) \mathbb{T}^{-1}\right)^{2}\right)\right] \mathrm{d} x \\
&=\int_{\Omega} \varrho \mathbf{f} \cdot \mathbf{u} \mathrm{d} x, \quad t \in(0, T] .
\end{aligned}
$$

This (formal) energy identity will be the starting point for the development of the weak compactness argument leading to the proof of existence of a large-data global-in-time finite-energy weak solution to the compressible FENE-P system under consideration.

To this end, we make some preliminary observations. As $\log$ is a concave function on $\mathbb{R}_{>0}$ (whereby $-\log (1-y) \geq y$ for all $y<1)$, we deduce from $(1.8)$ that

$$
-\Pi(\mathbb{T}) \geq \frac{1}{2} \operatorname{tr}(\mathbb{T}-\mathfrak{a} \log \mathbb{T})-\frac{\mathfrak{b}}{2} .
$$

Let us denote by $\mathfrak{l}_{\kappa}, \kappa=1, \ldots, d$, the eigenvalues of the symmetric positive definite matrix $\mathbb{T}$. Then, as $\mathfrak{a}>0$, it follows that

$$
\operatorname{tr}(\mathbb{T}-\mathfrak{a} \log \mathbb{T})=\sum_{\kappa=1}^{d}\left(\mathfrak{l}_{\kappa}-\mathfrak{a} \log \mathfrak{l}_{\kappa}\right) \geq d \min _{y>0}(y-\mathfrak{a} \log y)=d(\mathfrak{a}-\mathfrak{a} \log \mathfrak{a}) .
$$

Hence, for any $\mathfrak{a}>0$, we have that

$$
-\Pi(\mathbb{T})+\frac{d}{2} \mathfrak{a} \log \mathfrak{a}+\frac{\mathfrak{b}}{2} \geq \frac{1}{2}[\operatorname{tr}(\mathbb{T}-\mathfrak{a} \log \mathbb{T})+d \mathfrak{a} \log \mathfrak{a}] \geq \frac{d \mathfrak{a}}{2}>0 .
$$

Motivated by (3.20) and (3.22), for $t \in[0, T]$ we consider the following positive energy functional:

$$
E(t):=\int_{\Omega}\left[\frac{1}{2} \varrho|\mathbf{u}|^{2}+\frac{a}{\gamma-1} \varrho^{\gamma}-\Pi(\mathbb{T})+\frac{1}{2}(d \mathfrak{a} \log \mathfrak{a}+\mathfrak{b})\right] \mathrm{d} x .
$$


As $\gamma>\frac{d}{2}$ and $d \in\{2,3\}$, Hölder and Young inequalities then give

$$
\begin{aligned}
\int_{\Omega} \varrho \mathbf{f} \cdot \mathbf{u} \mathrm{d} x & \leq\|\mathbf{f}\|_{L^{\infty}\left((0, T] \times \Omega ; \mathbb{R}^{d}\right)}\|\sqrt{\varrho}|\mathbf{u}|\|_{L^{2}\left(\Omega ; \mathbb{R}^{d}\right)}\|\sqrt{\varrho}\|_{L^{2}(\Omega)} \\
& \leq C(\gamma)\|\mathbf{f}\|_{L^{\infty}\left((0, T] \times \Omega ; \mathbb{R}^{d}\right)}\|\sqrt{\varrho}|\mathbf{u}|\|_{L^{2}\left(\Omega ; \mathbb{R}^{d}\right)}\left(\int_{\Omega}\left(1+\rho^{\gamma}\right) \mathrm{d} x\right)^{\frac{1}{2}} \\
& \leq C(\mathbf{f}, \gamma, a, \mathfrak{a}) E(t), \quad t \in(0, T] .
\end{aligned}
$$

Thus, integrating (3.20) over the time interval $[0, t]$ with respect to the temporal variable and noting (3.13) implies that

$$
\begin{aligned}
& E(t)+\int_{0}^{t} \int_{\Omega}\left[\mu^{S}\right.\left.\left|\frac{\nabla_{x} \mathbf{u}+\nabla_{x}^{\mathrm{T}} \mathbf{u}}{2}-\frac{1}{d}\left(\operatorname{div}_{x} \mathbf{u}\right) \mathbb{I}\right|^{2}+\mu^{B}\left|\operatorname{div}_{x} \mathbf{u}\right|^{2}\right] \mathrm{d} x \mathrm{~d} t^{\prime}+\frac{1}{4 \lambda} \int_{0}^{t} \int_{\Omega} \operatorname{tr}\left((\mathbb{A}(\mathbb{T}))^{2} \mathbb{T}^{-1}\right) \mathrm{d} x \mathrm{~d} t^{\prime} \\
& \quad+\frac{\varepsilon}{2} \int_{0}^{t} \int_{\Omega}\left[\mathfrak{b}\left|\nabla_{x} \log \left(1-\frac{\operatorname{tr}(\mathbb{T})}{\mathfrak{b}}\right)\right|^{2}+\frac{\mathfrak{a}}{d}\left|\nabla_{x} \operatorname{tr}(\log \mathbb{T})\right|^{2}\right] \mathrm{d} x \mathrm{~d} t^{\prime} \\
& \leq E_{0}+ \int_{0}^{t} \int_{\Omega} \varrho \mathbf{f} \cdot \mathbf{u} \mathrm{d} x \mathrm{~d} t^{\prime} \\
& \leq E_{0}+C(\mathbf{f}, \gamma, a, \mathfrak{a}) \int_{0}^{t} E\left(t^{\prime}\right) \mathrm{d} t^{\prime}, \quad t \in(0, T]
\end{aligned}
$$

By recalling (1.8) and (3.1), the initial positive energy

$$
\begin{aligned}
E_{0} & :=\int_{\Omega}\left[\frac{1}{2} \varrho_{0}\left|\mathbf{u}_{0}\right|^{2}+\frac{a}{\gamma-1} \varrho_{0}^{\gamma}-\Pi\left(\mathbb{T}_{0}\right)+\frac{1}{2}(d \mathfrak{a} \log \mathfrak{a}+\mathfrak{b})\right] \mathrm{d} x \\
& \leq \int_{\Omega}\left[\frac{1}{2} \varrho_{0}\left|\mathbf{u}_{0}\right|^{2}+\frac{a}{\gamma-1} \varrho_{0}^{\gamma}+\frac{d \mathfrak{a}}{2} \log \left(\frac{\mathfrak{a}}{\tau_{\min }}\right)-\frac{\mathfrak{b}}{2} \log \left(1-\frac{\mathfrak{b}^{\star}}{\mathfrak{b}}\right)\right] \mathrm{d} x
\end{aligned}
$$

is finite. Thus, Gronwall's inequality implies that

$$
\begin{aligned}
& E(t)+\int_{0}^{t} \int_{\Omega}\left[\mu^{S}\right.\left.\left|\frac{\nabla_{x} \mathbf{u}+\nabla_{x}^{\mathrm{T}} \mathbf{u}}{2}-\frac{1}{d}\left(\operatorname{div}_{x} \mathbf{u}\right) \mathbb{I}\right|^{2}+\mu^{B}\left|\operatorname{div}_{x} \mathbf{u}\right|^{2}\right] \mathrm{d} x \mathrm{~d} t^{\prime}+\frac{1}{4 \lambda} \int_{0}^{t} \int_{\Omega} \operatorname{tr}\left((\mathbb{A}(\mathbb{T}))^{2} \mathbb{T}^{-1}\right) \mathrm{d} x \mathrm{~d} t^{\prime} \\
&+\frac{\varepsilon}{2} \int_{0}^{t} \int_{\Omega}\left[\mathfrak{b}\left|\nabla_{x} \log \left(1-\frac{\operatorname{tr}(\mathbb{T})}{\mathfrak{b}}\right)\right|^{2}+\frac{\mathfrak{a}}{d}\left|\nabla_{x} \operatorname{tr}(\log \mathbb{T})\right|^{2}\right] \mathrm{d} x \mathrm{~d} t^{\prime} \\
& \leq \mathrm{e}^{C(\mathbf{f}, \gamma, a, \mathfrak{a}) t} E_{0}, \quad t \in(0, T]
\end{aligned}
$$

Next we recall Korn's inequality (see, for example, [8]):

$$
\left\|\nabla_{x} \mathbf{v}\right\|_{L^{2}\left(\Omega ; \mathbb{R}^{d \times d}\right)} \leq C(d, \Omega)\left\|\frac{\nabla_{x} \mathbf{v}+\nabla_{x}^{\mathrm{T}} \mathbf{v}}{2}-\frac{1}{d}\left(\operatorname{div}_{x} \mathbf{v}\right) \mathbb{I}\right\|_{L^{2}\left(\Omega ; \mathbb{R}^{d \times d}\right)} \quad \forall \mathbf{v} \in W_{0}^{1,2}\left(\Omega, \mathbb{R}^{d}\right) .
$$

Thus we deduce the following (formal) inclusions from the a priori inequality (3.27):

$$
\begin{aligned}
& \varrho \in L^{\infty}\left(0, T ; L^{\gamma}(\Omega)\right), \quad \mathbf{u} \in L^{2}\left(0, T ; W_{0}^{1,2}\left(\Omega ; \mathbb{R}^{d}\right)\right), \quad \varrho|\mathbf{u}|^{2} \in L^{\infty}\left(0, T ; L^{1}(\Omega)\right), \\
& \Pi(\mathbb{T}) \in L^{\infty}\left(0, T ; L^{1}(\Omega)\right), \quad \operatorname{tr}\left((\mathbb{A}(\mathbb{T}))^{2} \mathbb{T}^{-1}\right) \in L^{1}\left(0, T ; L^{1}(\Omega)\right), \\
& \nabla_{x} \operatorname{tr}(\log \mathbb{T}) \in L^{2}\left(0, T ; L^{2}\left(\Omega ; \mathbb{R}^{d}\right)\right), \quad \nabla_{x} \log \left(1-\frac{\operatorname{tr}(\mathbb{T})}{\mathfrak{b}}\right) \in L^{2}\left(0, T ; L^{2}(\Omega)\right) .
\end{aligned}
$$

Of course in establishing these bounds we have assumed that $\mathbb{T}>0$ and $\operatorname{tr}(\mathbb{T})<\mathfrak{b}$ in $[0, T] \times \bar{\Omega} ;$ hence, $\mathbb{T} \in$ $L^{\infty}\left(0, T ; L^{\infty}\left(\Omega ; \mathbb{R}^{d \times d}\right)\right)$.

3.2. Further bounds on $\mathbb{T}$. In this section we will show that one can establish stronger bounds on $\mathbb{T}$ than those stated in (3.29). First, it follows from (1.8), (3.29) 3 and a Poincaré inequality that

$$
(I-f) \Pi(\mathbb{T}) \in L^{2}\left(0, T ; L^{2}(\Omega)\right),
$$

where $f \eta:=\frac{1}{|\Omega|} \int_{\Omega} \eta \mathrm{d} x$. Next, on assuming that $\mathbb{T}>0$ and $\operatorname{tr}(\mathbb{T})<\mathfrak{b}$ in $[0, T] \times \bar{\Omega}$ and $(3.29)_{2}$, we have that

$$
\|\mathbb{A}(\mathbb{T})\|_{L^{2}\left(0, T ; L^{2}\left(\Omega, \mathbb{R}^{d \times d}\right)\right)}^{2}=\int_{0}^{T} \int_{\Omega}\left|\mathbb{A}(\mathbb{T}) \mathbb{T}^{-\frac{1}{2}} \mathbb{T}^{\frac{1}{2}}\right|^{2} \mathrm{~d} x \mathrm{~d} t \leq b \int_{0}^{T} \int_{\Omega} \operatorname{tr}\left((\mathbb{A}(\mathbb{T}))^{2} \mathbb{T}^{-1}\right) \mathrm{d} x \mathrm{~d} t .
$$


By recalling (1.7), we take the inner product of (1.3) with $\mathbb{T}$, integrate over $\Omega$ with respect to $x$ and perform partial integration noting the boundary conditions (1.9), (1.10). Direct calculations imply that

$$
\begin{aligned}
\frac{1}{2} \frac{\mathrm{d}}{\mathrm{d} t} \int_{\Omega}|\mathbb{T}|^{2} \mathrm{~d} x+ & \varepsilon \int_{\Omega}\left|\nabla_{x} \mathbb{T}\right|^{2} \mathrm{~d} x \\
& =-\frac{1}{2} \int_{\Omega}\left(\mathbf{u} \cdot \nabla_{x}\right)|\mathbb{T}|^{2} \mathrm{~d} x+\int_{\Omega}\left(\nabla_{x} \mathbf{u} \mathbb{T}+\mathbb{T} \nabla_{x}^{\mathrm{T}} \mathbf{u}\right): \mathbb{T} \mathrm{d} x-\frac{1}{2 \lambda} \int_{\Omega} \mathbb{A}(\mathbb{T}): \mathbb{T} \mathrm{d} x \\
& \leq \frac{1}{2} \int_{\Omega}\left(\operatorname{div}_{x} \mathbf{u}\right)|\mathbb{T}|^{2} \mathrm{~d} x+2 \int_{\Omega}\left|\nabla_{x} \mathbf{u}\right||\mathbb{T}|^{2} \mathrm{~d} x+\frac{1}{2 \lambda} \int_{\Omega}|\mathbb{A}(\mathbb{T})||\mathbb{T}| \mathrm{d} x \\
& \leq C(\mathfrak{b}, \lambda) \int_{\Omega}\left[\left|\nabla_{x} \mathbf{u}\right|+|\mathbb{A}(\mathbb{T})|\right] \mathrm{d} x, \quad t \in(0, T],
\end{aligned}
$$

where once again we have assumed that $\mathbb{T}>0$ and $\operatorname{tr}(\mathbb{T})<\mathfrak{b}$ in $[0, T] \times \bar{\Omega}$ for the last inequality. Thus, integrating (3.32) over $t \in(0, T)$ and noting (3.29) $)_{1,2}$ and (3.31), we can supplement (3.29)-(3.31) with the inclusion:

$$
\mathbb{T} \in L^{2}\left(0, T ; W^{1,2}\left(\Omega ; \mathbb{R}^{d \times d}\right)\right) .
$$

In the case of the Oldroyd-B model, we are only able to establish (3.33) for $d=2$, as there we have to use a GagliardoNirenberg inequality in (3.32), as we do not have that $\operatorname{tr}(\mathbb{T})<\mathfrak{b}$ and hence $\mathbb{T} \in L^{\infty}\left(0, T ; L^{\infty}\left(\Omega ; \mathbb{R}^{d \times d}\right)\right)$ is not guaranteed. This restricts the proof of the existence of a global-in-time weak solution for compressible Oldroyd-B model to the case of $d=2$; see [3] for the details. In fact, this is also true in the incompressible case; see [1].

Motivated by these formal calculations, we shall now embark on a rigorous argument aimed at proving the existence of global-in-time weak solutions to the compressible FENE-P model for $d=2$ and $d=3$.

\section{Weak solutions, main Results and the COnStruCtion of approximating SOlutions}

The rest of the paper is devoted to the proof of the existence of large-data global-in-time finite-energy weak solutions to the compressible FENE-P model (1.1)-(1.10), (3.1) for $d=2$ and 3.

4.1. Weak solutions and main results. Our main result is the proof of the existence of large-data global-in-time finite-energy weak solutions to the compressible FENE-P model. First of all, we give the definition of a finite-energy weak solution to (1.1)-(1.10), (3.1). For the definition of $C_{w}([0, T], X)$, where $X$ is a Banach space, see Section 5.4 below.

Definition 4.1. Let $T>0$ and let $\Omega \subset \mathbb{R}^{d}, d=2$ or 3 , be a bounded $C^{2, \beta}$ domain, with $0<\beta<1$. Let $\mathbf{f} \in$ $L^{\infty}\left((0, T] \times \Omega ; \mathbb{R}^{d}\right)$. We say that $(\varrho, \mathbf{u}, \mathbb{T})$ is a finite-energy weak solution in $(0, T] \times \Omega$ to the system of equations (1.1)-(1.10), supplemented by the initial data (3.1), if:

- $\varrho \geq 0$ a.e. in $(0, T] \times \Omega, \quad \varrho \in C_{w}\left([0, T] ; L^{\gamma}(\Omega)\right), \quad \mathbf{u} \in L^{2}\left(0, T ; W_{0}^{1,2}\left(\Omega ; \mathbb{R}^{d}\right)\right), \quad \mathbb{T}$ is symmetric,

$$
\begin{aligned}
& \varrho \mathbf{u} \in C_{w}\left([0, T] ; L^{\frac{2 \gamma}{\gamma+1}}\left(\Omega ; \mathbb{R}^{d}\right)\right), \quad \varrho|\mathbf{u}|^{2} \in L^{\infty}\left(0, T ; L^{1}(\Omega)\right), \quad \mathbb{T}>0 \text { and } \operatorname{tr}(\mathbb{T})<\mathfrak{b} \text { a.e. in }(0, T] \times \Omega, \\
& \mathbb{T} \in C_{w}\left([0, T] ; L^{2}\left(\Omega ; \mathbb{R}^{d \times d}\right)\right) \cap L^{2}\left(0, T ; W^{1,2}\left(\Omega ; \mathbb{R}^{d \times d}\right)\right), \\
& \Pi(\mathbb{T}) \in L^{\infty}\left(0, T ; L^{1}(\Omega)\right), \quad(I-f) \Pi(\mathbb{T}) \in L^{2}\left(0, T ; L^{2}(\Omega)\right), \\
& \operatorname{tr}\left((\mathbb{A}(\mathbb{T}))^{2} \mathbb{T}^{-1}\right) \in L^{1}\left(0, T ; L^{1}(\Omega)\right), \quad \mathbb{A}(\mathbb{T}) \in L^{2}\left(0, T ; L^{2}\left(\Omega ; \mathbb{R}^{d \times d}\right)\right), \\
& \nabla_{x} \operatorname{tr}(\log \mathbb{T}) \in L^{2}\left(0, T ; L^{2}\left(\Omega ; \mathbb{R}^{d}\right)\right), \quad \nabla_{x} \log \left(1-\frac{\operatorname{tr}(\mathbb{T})}{\mathfrak{b}}\right) \in L^{2}\left(0, T ; L^{2}(\Omega)\right) .
\end{aligned}
$$

- For any $t \in(0, T]$ and any test function $\phi \in C^{\infty}([0, T] \times \bar{\Omega})$, one has

$$
\int_{0}^{t} \int_{\Omega}\left[\varrho \partial_{t} \phi+\varrho \mathbf{u} \cdot \nabla_{x} \phi\right] \mathrm{d} x \mathrm{~d} t^{\prime}=\int_{\Omega} \varrho(t, \cdot) \phi(t, \cdot) \mathrm{d} x-\int_{\Omega} \varrho_{0} \phi(0, \cdot) \mathrm{d} x,
$$

- For any $t \in(0, T]$ and any test function $\varphi \in C^{\infty}\left([0, T] ; C_{0}^{\infty}\left(\Omega ; \mathbb{R}^{d}\right)\right)$, one has

$$
\begin{aligned}
\int_{0}^{t} \int_{\Omega}\left[\varrho \mathbf{u} \cdot \partial_{t} \boldsymbol{\varphi}+(\varrho \mathbf{u} \otimes \mathbf{u}): \nabla_{x} \boldsymbol{\varphi}+p(\varrho) \operatorname{div}_{x} \boldsymbol{\varphi}-\mathbb{S}\left(\nabla_{x} \mathbf{u}\right): \nabla_{x} \boldsymbol{\varphi}\right] \mathrm{d} x \mathrm{~d} t^{\prime} \\
\quad=\int_{0}^{t} \int_{\Omega}\left[\mathbb{A}(\mathbb{T}): \nabla_{x} \boldsymbol{\varphi}-\Pi(\mathbb{T}) \operatorname{div}_{x} \boldsymbol{\varphi}-\varrho \mathbf{f} \cdot \boldsymbol{\varphi}\right] \mathrm{d} x \mathrm{~d} t^{\prime}+\int_{\Omega} \varrho \mathbf{u}(t, \cdot) \cdot \boldsymbol{\varphi}(t, \cdot) \mathrm{d} x-\int_{\Omega} \varrho_{0} \mathbf{u}_{0} \cdot \boldsymbol{\varphi}(0, \cdot) \mathrm{d} x
\end{aligned}
$$


- For any $t \in(0, T]$ and any test function $\mathbb{Y} \in C^{\infty}\left([0, T] \times \bar{\Omega} ; \mathbb{R}^{d \times d}\right)$, one has

$$
\begin{gathered}
\int_{0}^{t} \int_{\Omega}\left[\mathbb{T}: \partial_{t} \mathbb{Y}-\left(\mathbf{u} \cdot \nabla_{x}\right) \mathbb{T}: \mathbb{Y}+\left(\nabla_{x} \mathbf{u} \mathbb{T}+\mathbb{T} \nabla_{x}^{\mathrm{T}} \mathbf{u}\right): \mathbb{Y}-\varepsilon \nabla_{x} \mathbb{T}:: \nabla_{x} \mathbb{Y}\right] \mathrm{d} x \mathrm{~d} t^{\prime} \\
=\int_{0}^{t} \int_{\Omega} \frac{1}{2 \lambda} \mathbb{A}(\mathbb{T}): \mathbb{Y} \mathrm{d} x \mathrm{~d} t^{\prime}+\int_{\Omega} \mathbb{T}(t, \cdot): \mathbb{Y}(t, \cdot) \mathrm{d} x-\int_{\Omega} \mathbb{T}_{0}: \mathbb{Y}(0, \cdot) \mathrm{d} x
\end{gathered}
$$

where the term involving the notation $::$ is

$$
\nabla_{x} \mathbb{T}:: \nabla_{x} \mathbb{Y}=\sum_{\kappa=1}^{d} \partial_{x_{\kappa}} \mathbb{T}: \partial_{x_{\kappa}} \mathbb{Y} .
$$

- The continuity equation holds in the sense of renormalised solutions:

$$
\partial_{t} b(\varrho)+\operatorname{div}_{x}(b(\varrho) \mathbf{u})+\left(b^{\prime}(\varrho) \varrho-b(\varrho)\right) \operatorname{div}_{x} \mathbf{u}=0 \quad \text { in } C_{0}^{\infty}((0, T) \times \Omega)^{\prime},
$$

for any $b \in C([0, \infty)) \cap C^{1}((0, \infty))$ such that

$$
\left|b^{\prime}(s)\right|<C s^{-\mathfrak{m}_{0}} \quad \forall s \in(0,1] \quad \text { and } \quad\left|b^{\prime}(s)\right|<C s^{\mathfrak{m}_{1}} \quad \forall s \geq 1,
$$

where $\mathfrak{m}_{0}<1$ and $\mathfrak{m}_{1} \in(-1, \infty)$; see (6.2.9) and (6.2.10) in [22].

- For a.e. $t \in(0, T]$ the following energy inequality holds:

$$
\begin{aligned}
E(t) & +\int_{0}^{t} \int_{\Omega}\left[\mu^{S}\left|\frac{\nabla_{x} \mathbf{u}+\nabla_{x}^{\mathrm{T}} \mathbf{u}}{2}-\frac{1}{d}\left(\operatorname{div}_{x} \mathbf{u}\right) \mathbb{I}\right|^{2}+\mu^{B}\left|\operatorname{div}_{x} \mathbf{u}\right|^{2}\right] \mathrm{d} x \mathrm{~d} t^{\prime} \\
& +\frac{1}{4 \lambda} \int_{0}^{t} \int_{\Omega} \operatorname{tr}\left((\mathbb{A}(\mathbb{T}))^{2} \mathbb{T}^{-1}\right) \mathrm{d} x \mathrm{~d} t^{\prime}+\frac{\varepsilon}{2} \int_{0}^{t} \int_{\Omega}\left[\mathfrak{b}\left|\nabla_{x} \log \left(1-\frac{\operatorname{tr}(\mathbb{T})}{\mathfrak{b}}\right)\right|^{2}+\frac{\mathfrak{a}}{d}\left|\nabla_{x} \operatorname{tr}(\log \mathbb{T})\right|^{2}\right] \mathrm{d} x \mathrm{~d} t^{\prime} \\
\leq & E_{0}+\int_{0}^{t} \int_{\Omega} \varrho \mathbf{f} \cdot \mathbf{u} \mathrm{d} x \mathrm{~d} t^{\prime} \\
\leq & \mathrm{e}^{C(\mathbf{f}, \gamma, a, \mathfrak{a}) t} E_{0},
\end{aligned}
$$

where $E(t)$ and $E_{0}$ are defined by (3.23) and (3.26). We note that $E_{0}$ is finite under the assumptions (3.1) on the initial data.

Remark 4.2. Definition 4.1 is fairly standard. The energy inequality (4.8) identifies an important class of weak solutions, usually termed dissipative or finite-energy weak solutions. We note that, given a smooth solution satisfying $\varrho \geq 0, \mathbb{T}>0$ and $\operatorname{tr}(\mathbb{T})<\mathfrak{b}$ in $[0, T] \times \bar{\Omega}$, the energy inequality (4.8) can be derived by integrating the a priori bound (3.20) over $[0, t]$ with respect to the temporal variable, by noting (3.23), (3.26) and using Lemma 3.1, to obtain (3.25) and (3.27).

Remark 4.3. In addition to the energy inequality (4.8), one can derive the following inequality:

$$
\frac{1}{2} \int_{\Omega}|\mathbb{T}(t)|^{2} \mathrm{~d} x+\varepsilon \int_{0}^{t} \int_{\Omega}\left|\nabla_{x} \mathbb{T}\right|^{2} \mathrm{~d} x \mathrm{~d} t^{\prime} \leq \frac{1}{2} \int_{\Omega}\left|\mathbb{T}_{0}\right|^{2} \mathrm{~d} x+C(\mathbf{f}, \gamma, a, \mathfrak{a}, \mathfrak{b}, \lambda), \quad \text { for a.e. } t \in(0, T] .
$$

We note that, given a smooth solution satisfying $\varrho \geq 0, \mathbb{T}>0$ and $\operatorname{tr}(\mathbb{T})<\mathfrak{b}$ in $[0, T] \times \bar{\Omega}$, the energy inequality (4.9) can be derived by integrating the a priori bound (3.32) over $[0, t]$ with respect to the temporal variable, by noting $\mathbf{u} \in L^{2}\left(0, T ; W_{0}^{1,2}\left(\Omega, \mathbb{R}^{d}\right)\right)$ and $\mathbb{A}(\mathbb{T}) \in L^{2}\left(0, T ; L^{2}\left(\Omega, \mathbb{R}^{d \times d}\right)\right)$, which can be derived from (4.8), (3.31) and (3.29) 2 .

We are now ready to state our first main theorem, which asserts the global-in-time existence of large-data finite-energy weak solutions to the compressible FENE-P model.

Theorem 4.4. There exists a finite-energy weak solution $(\varrho, \mathbf{u}, \mathbb{T})$ to the compressible FENE-P model (1.1)-(1.10) with initial data (3.1), in the sense of Definition 4.1.

The proof of Theorem 4.4 involves four levels of approximation, which are described in Section 6; the respective passages to the limits with the four levels of approximation are carried out in Sections 7-11. Before embarking on the technical part of the paper, we recall, in Section 5 , a number of preliminary results, which will be required in the proofs.

\section{Preliminaries}

In this section we recall some technical tools that will be required in the rest of the paper. 
5.1. Classical mollifier. This is required to approximate the original initial data, (3.1), with smooth initial data in order to apply classical existence results for parabolic systems. Let $\zeta \in C^{\infty}\left(\mathbb{R}^{d}\right)$ be a nonnegative, radially symmetric function such that

We define the mollification kernel

$$
\operatorname{supp} \zeta \subset B(0,1), \quad \int_{\mathbb{R}^{d}} \zeta(x) \mathrm{d} x=1 .
$$

$$
\zeta_{\theta}(\cdot)=\frac{1}{\theta^{d}} \zeta(\cdot \bar{\theta}), \quad \text { for any } \theta>0 .
$$

For any locally integrable function $v$ defined on $\mathbb{R}^{d}$ with values in a Banach space $X$, we define the classical (Friedrichs) mollifier $S_{\theta}$ as the following convolution operator:

$$
S_{\theta}[v](x):=\left(\zeta_{\theta} * v\right)(x)=\int_{\mathbb{R}^{d}} \zeta_{\theta}(x-y) v(y) \mathrm{d} y .
$$

Some of the key properties of $S_{\theta}$ are summarised in the next lemma.

Lemma 5.1 (Theorem 10.1 in [12]). Let $X$ be a Banach space. If $v \in L_{\mathrm{loc}}^{1}\left(\mathbb{R}^{d} ; X\right)$, we have that $S_{\theta}[v] \in C^{\infty}\left(\mathbb{R}^{d} ; X\right)$. In addition, the following hold:

(i) If $v \in L^{q}\left(\mathbb{R}^{d} ; X\right), 1 \leq q<\infty$, then

$$
\left\|S_{\theta}[v]\right\|_{L^{q}\left(\mathbb{R}^{d} ; X\right)} \leq\|v\|_{L^{q}\left(\mathbb{R}^{d} ; X\right)} ; \quad S_{\theta}[v] \rightarrow v \quad \text { strongly in } L^{q}\left(\mathbb{R}^{d} ; X\right), \quad \text { as } \theta \rightarrow 0 .
$$

(ii) If $v \in L^{\infty}\left(\mathbb{R}^{d} ; X\right)$, then

$$
\left\|S_{\theta}[v]\right\|_{L^{\infty}\left(\mathbb{R}^{d} ; X\right)} \leq\|v\|_{L^{\infty}\left(\mathbb{R}^{d} ; X\right)} .
$$

5.2. The Bogovskiı̆ operator. We recall the Bogovskil operator, which is required to prove higher order integrability of the density. The construction can be found in [7] and in Chapter III of Galdi's book [14]; see also Lemma 3.17 in $[22]$.

Lemma 5.2. Let $1<q<\infty$ and suppose that $G \subset \mathbb{R}^{d}$ is a bounded Lipschitz domain. Let $L_{0}^{q}(G)$ be the space of all $L^{q}(G)$ functions with zero integral mean value. Then, there exists a linear operator $\mathcal{B}_{G}$ from $L_{0}^{q}(G)$ to $W_{0}^{1, q}\left(G ; \mathbb{R}^{d}\right)$, called the Bogovskiu operator, such that for any $\rho \in L_{0}^{q}(G)$ one has

$$
\operatorname{div}_{x} \mathcal{B}_{G}(\rho)=\rho \quad \text { in } G ; \quad\left\|\mathcal{B}_{G}(\rho)\right\|_{W_{0}^{1, q}\left(G ; \mathbb{R}^{d}\right)} \leq c(q, d, G)\|\rho\|_{L^{q}(G)} .
$$

If, in addition, $\rho=\operatorname{div}_{x} \mathbf{g}$ for some $\mathbf{g} \in L^{s}\left(G ; \mathbb{R}^{d}\right), 1<s<\infty, \mathbf{g} \cdot \nu_{G}=0$ on $\partial G$, where $\nu_{G}$ is normal to $\partial G$, then the following inequality holds:

$$
\left\|\mathcal{B}_{G}(\rho)\right\|_{L^{s}\left(G ; \mathbb{R}^{d}\right)} \leq c(d, s, G)\|\mathbf{g}\|_{L^{s}\left(G ; \mathbb{R}^{d}\right)} .
$$

5.3. Compactness theorems. We begin by recalling the following result, usually referred to as the Aubin-LionsSimon compactness theorem (see Simon [24]).

Lemma 5.3. Let $X_{0}, X$ and $X_{1}$ be three Banach spaces with $X_{0} \subset X \subset X_{1}$. Suppose that $X_{0} \hookrightarrow \hookrightarrow X$, i.e., $X_{0}$ is compactly embedded in $X$, and that $X \hookrightarrow X_{1}$, i.e., $X$ is continuously embedded in $X_{1}$. For $1 \leq q, s \leq \infty$, let

$$
Y=\left\{v \in L^{q}\left(0, T ; X_{0}\right): \partial_{t} v \in L^{s}\left(0, T ; X_{1}\right)\right\} .
$$

Then, the following properties hold:

(i) If $q<\infty$, then the embedding of $Y$ into $L^{q}(0, T ; X)$ is compact;

(ii) If $q=\infty$ and $s>1$, then the embedding of $Y$ into $C([0, T] ; X)$ is compact.

5.4. On $C_{w}([0, T] ; X)$ type spaces. Let $X$ be a Banach space. We denote by $C_{w}([0, T] ; X)$ the set of all functions $v \in L^{\infty}(0, T ; X)$ such that the mapping $t \in[0, T] \mapsto\langle\phi, v(t)\rangle_{X} \in \mathbb{R}$ is continuous on $[0, T]$ for all $\phi \in X^{\prime}$. Here and throughout the paper, we use $X^{\prime}$ to denote the dual space of $X$, and $\langle\cdot, \cdot\rangle_{X}$ to denote the duality pairing between $X^{\prime}$ and $X$. We recall the following lemma, see Strauss [25] (cf. Lions \& Magenes [19], Lemma 8.1, Ch. 3, Sec. 8.4) for a proof.

Lemma 5.4. Suppose that $X$ and $Y$ are Banach spaces. Assume that the space $X$ is reflexive and is continuously embedded in the space $Y$; then,

$$
L^{\infty}(0, T ; X) \cap C_{w}([0, T] ; Y)=C_{w}([0, T] ; X) .
$$

We recall the following Arzelà-Ascoli type result, and refer to Lemma 6.2 in [22] for its proof.

Lemma 5.5. Let $q, s \in(1, \infty)$ and let $G$ be a bounded Lipschitz domain in $\mathbb{R}^{d}, d \geq 2$. Suppose that $\left(g_{n}\right)_{n \in \mathbb{N}}$ is a sequence of functions in $C_{w}\left([0, T] ; L^{s}(G)\right)$ such that $\left(g_{n}\right)_{n \in \mathbb{N}}$ is bounded in $C\left([0, T] ; W^{-1, q}(G)\right) \cap L^{\infty}\left(0, T ; L^{s}(G)\right)$, where $W^{-1, q}(G)$ is the dual of $W^{1, q}(G)$. Then, there exists a subsequence (not indicated) such that the following hold: 
(i) $g_{n} \rightarrow g$ in $C_{w}\left([0, T] ; L^{s}(G)\right)$;

(ii) If, in addition, $q \leq \frac{d}{d-1}$, or $q>\frac{d}{d-1}$ and $s>\frac{d q}{d+q}$, then $g_{n} \rightarrow g$ strongly in $C\left([0, T] ; W^{-1, q}(G)\right)$.

5.5. Regularity of the parabolic Neumann problem. We first introduce fractional-order Sobolev spaces. Let $G$ be the whole space $\mathbb{R}^{d}$ or a bounded Lipschitz domain in $\mathbb{R}^{d}$. For any $k \in \mathbb{N}, \beta \in(0,1)$ and $s \in[1, \infty)$, we define

$$
W^{k+\beta, s}(G):=\left\{v \in W^{k, s}(G):\|v\|_{W^{k+\beta, s}(G)}<\infty\right\},
$$

where

$$
\|v\|_{W^{k+\beta, s}(G)}:=\|v\|_{W^{k, s}(G)}+\sum_{|\alpha|=k}\left(\int_{G} \int_{G} \frac{\left|\partial^{\alpha} v(x)-\partial^{\alpha} v(y)\right|^{s}}{|x-y|^{d+\beta s}} \mathrm{~d} x \mathrm{~d} y\right)^{\frac{1}{s}} .
$$

The following classical results are taken from Section 7.6.1 in [22]. Let $G$ be a bounded domain in $\mathbb{R}^{d}$ and consider the parabolic initial-boundary-value problem:

$$
\begin{aligned}
\partial_{t} \rho-\varepsilon \Delta_{x} \rho & =h & & \text { in }(0, T] \times G, \\
\rho(0, \cdot) & =\rho_{0} & & \text { in } G, \\
\nu_{G} \cdot \nabla_{x} \rho & =0 & & \text { in }(0, T] \times \partial G .
\end{aligned}
$$

Here $\varepsilon>0, \rho_{0}$ and $h$ are known functions, $\nu_{G}$ is normal to $\partial G$ and $\rho$ is the unknown solution. The first regularity result of relevance to us here is encapsulated in the following lemma.

Lemma 5.6. Let $0<\beta<1,1<q, s<\infty$ and suppose that $G$ is a bounded domain in $\mathbb{R}^{d}$,

$$
G \in C^{2, \beta}, \quad \rho_{0} \in W_{\nu}^{2-\frac{2}{q}, s}(G), \quad h \in L^{q}\left(0, T ; L^{s}(G)\right),
$$

where $W_{\nu}^{2-\frac{2}{q}, s}(G)$ is the completion of the linear space $\left\{v \in C^{\infty}(\bar{G}):\left.\left(\nu_{G} \cdot \nabla_{x} v\right)\right|_{\partial G}=0\right\}$ in the norm of $W^{2-\frac{2}{q}, s}(G)$. Then, there exists a unique function $\rho$ satisfying

$$
\rho \in L^{q}\left(0, T ; W^{2, s}(G)\right) \cap C\left([0, T] ; W^{2-\frac{2}{q}, s}(G)\right), \quad \partial_{t} \rho \in L^{q}\left(0, T ; L^{s}(G)\right)
$$

and solving $(5.2)_{1}$ a.e. in $(0, T] \times G,(5.2)_{2}$ a.e. in $G$; in addition, $\rho$ satisfies $(5.2)_{3}$ in the sense of the normal trace, which is well defined since $\Delta_{x} \rho \in L^{q}\left(0, T ; L^{s}(G)\right)$. Moreover, we have that

$$
\begin{aligned}
& \varepsilon^{1-\frac{1}{q}}\|\rho\|_{L^{\infty}\left(0, T ; W^{2-\frac{2}{q}, s}(G)\right)}+\left\|\partial_{t} \rho\right\|_{L^{q}\left(0, T ; L^{s}(G)\right)}+\varepsilon\|\rho\|_{L^{q}\left(0, T ; W^{2, s}(G)\right)} \\
& \leq C(q, s, G)\left[\varepsilon^{1-\frac{1}{q}}\left\|\rho_{0}\right\|_{W^{2-\frac{2}{q}, s}(G)}+\|h\|_{L^{q}\left(0, T ; L^{s}(G)\right)}\right] .
\end{aligned}
$$

The second result that we state concerns parabolic problems with a divergence-form source term, $h=\operatorname{div}_{x} \mathbf{g}$.

Lemma 5.7. Let $0<\beta<1,1<q, s<\infty$ and suppose that $G$ is a bounded domain in $\mathbb{R}^{d}$,

$$
G \in C^{2, \beta}, \quad \rho_{0} \in L^{q}(G), \quad \mathrm{g} \in L^{q}\left(0, T ; L^{s}\left(G ; \mathbb{R}^{d}\right)\right) .
$$

Then, there exists a unique function $\rho \in L^{q}\left(0, T ; W^{1, s}(G)\right) \cap C\left([0, T] ; L^{s}(G)\right)$ satisfying $(5.2)_{2}$ a.e. in $G$ and

$$
\frac{\mathrm{d}}{\mathrm{d} t} \int_{G} \rho \phi \mathrm{d} x+\varepsilon \int_{G} \nabla_{x} \rho \cdot \nabla_{x} \phi \mathrm{d} x=-\int_{G} \mathbf{g} \cdot \nabla_{x} \phi \mathrm{d} x \quad \forall \phi \in C^{\infty}(\bar{\Omega}) \quad \text { in } C_{0}^{\infty}(0, T)^{\prime} .
$$

Moreover, we have that

$$
\varepsilon^{1-\frac{1}{q}}\|\rho\|_{L^{\infty}\left(0, T ; L^{s}(G)\right)}+\varepsilon\left\|\nabla_{x} \rho\right\|_{L^{q}\left(0, T ; L^{s}\left(G ; \mathbb{R}^{d}\right)\right)} \leq C(q, s, G)\left[\varepsilon^{1-\frac{1}{q}}\left\|\rho_{0}\right\|_{L^{s}(G)}+\|\mathbf{g}\|_{L^{q}\left(0, T ; L^{s}\left(G ; \mathbb{R}^{d}\right)\right)}\right] .
$$

5.6. Effective viscous flux. A key step in passing to the limit in a sequence of approximations to the compressible Navier-Stokes system is the proof of strong convergence of the approximations to the density, based on weak convergence of the, so called, effective viscous flux. A helpful tool in the proof of this is Lemma 7.36 in [22]. However, due to the presence of the extra stress tensor $\mathbb{A}(\mathbb{T})$ and the polymeric pressure $\Pi(\mathbb{T})$ on the right-hand side of $(1.2)$, we require an extension of this result, see Lemma 5.6 in [4] for $d=3$ and Lemma 2.3 in [5] for $d=2$. Below, we combine these lemmas to obtain an extension valid for $d=2$ and 3 .

First, though, we introduce the inverse divergence operator $\mathfrak{A}$, which is a pseudodifferential operator defined via the Fourier transform $\mathfrak{F}$. With

$$
\mathfrak{S}\left(\mathbb{R}^{d}\right):=\left\{\eta \in C^{\infty}\left(\mathbb{R}^{d}\right): \sup _{x \in \mathbb{R}^{d}}\left|x_{1}^{\varsigma_{1}} \cdots x_{d}^{\varsigma_{d}}\left(\partial_{x_{1}}^{\kappa_{1}} \ldots \partial_{x_{d}}^{\kappa_{d}}\right) \eta\right| \leq C(|\varsigma|,|\kappa|) \quad \forall \varsigma, \kappa \in \mathbb{N}^{d}\right\},
$$

the space of smooth rapidly decreasing (complex-valued) functions, we introduce the Fourier transform $\mathfrak{F}: \mathfrak{S}\left(\mathbb{R}^{d}\right) \rightarrow$ $\mathfrak{S}\left(\mathbb{R}^{d}\right)$, and its inverse $\mathfrak{F}^{-1}: \mathfrak{S}\left(\mathbb{R}^{d}\right) \rightarrow \mathfrak{S}\left(\mathbb{R}^{d}\right)$, defined by

$$
[\mathfrak{F}(\eta)](y)=\frac{1}{(2 \pi)^{\frac{d}{2}}} \int_{\mathbb{R}^{d}} \mathrm{e}^{-i x \cdot y} \eta(x) \mathrm{d} x \quad \text { and } \quad\left[\mathfrak{F}^{-1}(\eta)\right](x)=\frac{1}{(2 \pi)^{\frac{d}{2}}} \int_{\mathbb{R}^{d}} \mathrm{e}^{i x \cdot y} \eta(y) \mathrm{d} y .
$$


These are extended to $\mathfrak{F}, \mathfrak{F}^{-1}: \mathfrak{S}\left(\mathbb{R}^{d}\right)^{\prime} \rightarrow \mathfrak{S}\left(\mathbb{R}^{d}\right)^{\prime}$, where $\mathfrak{S}\left(\mathbb{R}^{d}\right)^{\prime}$, the dual of $\mathfrak{S}\left(\mathbb{R}^{d}\right)$, is the space of tempered distributions, via

$$
\langle\mathfrak{F}(\eta), \xi\rangle_{\mathfrak{S}\left(\mathbb{R}^{d}\right)}=\langle\eta, \mathfrak{F}(\xi)\rangle_{\mathfrak{S}\left(\mathbb{R}^{d}\right)} \quad \text { and } \quad\left\langle\mathfrak{F}^{-1}(\eta), \xi\right\rangle_{\mathfrak{S}\left(\mathbb{R}^{d}\right)}=\left\langle\eta, \mathfrak{F}^{-1}(\xi)\right\rangle_{\mathfrak{S}\left(\mathbb{R}^{d}\right)} \quad \forall \xi \in \mathfrak{S}\left(\mathbb{R}^{d}\right) .
$$

Next we define the inverse divergence operator $\mathfrak{A}: \mathfrak{S}\left(\mathbb{R}^{d}\right) \rightarrow\left(\left[\mathfrak{S}\left(\mathbb{R}^{d}\right)\right]^{d}\right)^{\prime}$ with components $\mathfrak{A}_{j}: \mathfrak{S}\left(\mathbb{R}^{d}\right) \rightarrow \mathfrak{S}\left(\mathbb{R}^{d}\right)^{\prime}$, $j=1, \ldots, d$, defined by

$$
\mathfrak{A}_{j}(\eta):=-\mathfrak{F}^{-1}\left(\frac{i y_{j}}{|y|^{2}}[\mathfrak{F}(\eta)](y)\right) .
$$

On noting the Marcinkiewicz theorem about multipliers and Sobolev embedding, the operator $\mathfrak{A}_{j}$ can be extended to $\mathfrak{A}_{j}: L^{r}\left(\mathbb{R}^{d}\right) \rightarrow L^{\frac{d r}{d-r}}\left(\mathbb{R}^{d}\right)$ for $r \in(1, d), j=1, \ldots, d$, see (4.4.3) in [22] for the details. Finally, we adopt the convention in the lemma below if $\mathfrak{A}$ is applied to a function defined on $\Omega \subset \mathbb{R}^{d}$ only, then the function is extended by zero from $\Omega$ to $\mathbb{R}^{d}$.

Lemma 5.8. Given $\left\{\left(g_{n}, \mathbf{u}_{n}, \mathbf{m}_{n}, p_{n}, \mathbb{B}_{n}, f_{n}, \mathbf{F}_{n}\right)\right\}_{n \in \mathbb{N}}$, we assume for any $\zeta \in C_{0}^{\infty}(\Omega)$ that, as $n \rightarrow \infty$,

$$
\begin{aligned}
g_{n} & \rightarrow g & & \text { in } C_{w}\left([0, T] ; L^{q}(\Omega)\right), \quad \text { weakly-* in } L^{\omega}\left(\Omega_{T}\right), \\
\mathbf{u}_{n} & \rightarrow \mathbf{u} & & \text { weakly in } L^{2}\left(0, T ; W_{0}^{1,2}\left(\Omega ; \mathbb{R}^{d}\right)\right), \\
\mathbf{m}_{n} & \rightarrow \mathbf{m} & & \text { in } C_{w}\left([0, T] ; L^{z}\left(\Omega, \mathbb{R}^{d}\right)\right), \\
p_{n} & \rightarrow p & & \text { weakly in } L^{r}\left(0, T ; L^{r}(\Omega)\right), \\
\mathbb{B}_{n} & \rightarrow \mathbb{B} & & \text { strongly in } L^{1}\left(0, T ; L^{\frac{q}{q-1}}\left(\Omega ; \mathbb{R}^{d \times d}\right)\right), \\
f_{n} & \rightarrow f & & \text { weakly in } L^{2}\left(0, T ; W^{-1,2}(\Omega)\right), \\
\mathfrak{A}\left(\zeta f_{n}\right) & \rightarrow \mathfrak{A}(\zeta f) & & \text { strongly in } L^{2}\left(0, T ; L^{\frac{z}{z-1}}\left(\Omega ; \mathbb{R}^{d}\right)\right), \\
\mathbf{F}_{n} & \rightarrow \mathbf{F} & & \text { weakly in } L^{s}\left(0, T ; L^{s}\left(\Omega, \mathbb{R}^{d}\right)\right),
\end{aligned}
$$

where $q \in(d, \infty), r, s \in(1, \infty), \omega \in\left[\max \left\{2, \frac{r}{r-1}\right\}, \infty\right]$ and $z \in\left(q^{\star}(d), \infty\right)$. Here $q^{\star}(d)=\frac{q}{q-1}$ if $d=2$ and $q^{\star}(d)=\frac{6 q}{5 q-6}$ if $d=3$.

In addition, suppose that

$$
\begin{aligned}
& \frac{\partial g_{n}}{\partial t}+\nabla_{x} \cdot\left(\mathbf{u}_{n} g_{n}\right)=f_{n} \quad \text { in } C_{0}^{\infty}((0, T) \times \Omega)^{\prime}, \\
& \frac{\partial \mathbf{m}_{n}}{\partial t}+\nabla_{x} \cdot\left(\mathbf{m}_{n} \otimes \mathbf{u}_{n}\right)-\mu_{1} \Delta_{x} \mathbf{u}_{n}-\left(\mu_{1}+\mu_{2}\right) \nabla_{x}\left(\nabla_{x} \cdot \mathbf{u}_{n}\right)+\nabla_{x} p_{n}=\mathbf{F}_{n}+\nabla_{x} \cdot \mathbb{B}_{n} \quad \text { in } C_{0}^{\infty}((0, T) \times \Omega)^{\prime},
\end{aligned}
$$

where $\mu_{1}, \mu_{2}$ are positive constants. It then follows that, for any $\zeta \in C_{0}^{\infty}(\Omega)$ and $\eta \in C_{0}^{\infty}(0, T)$,

$$
\lim _{n \rightarrow \infty} \int_{0}^{T} \eta\left(\int_{\Omega} \zeta g_{n}\left[p_{n}-\left(2 \mu_{1}+\mu_{2}\right) \nabla_{x} \cdot \mathbf{u}_{n}\right] \mathrm{d} x\right) \mathrm{d} t=\int_{0}^{T} \eta\left(\int_{\Omega} \zeta g\left[p-\left(2 \mu_{1}+\mu_{2}\right) \nabla_{x} \cdot \mathbf{u}\right] \mathrm{d} x\right) \mathrm{d} t .
$$

Moreover, by noting Corollary 5.1 in [4] for $d=3$ and Corollary 2.1 in [5] for $d=2$, we have the following corollary.

Corollary 5.9. The results of Lemma 5.8 hold with assumptions (5.7) 6,7 replaced by

$$
f_{n} \rightarrow f \quad \text { weakly in } L^{2}\left(0, T ; L^{2}(\Omega)\right), \quad \text { as } n \rightarrow \infty .
$$

\section{Definition of the SEQuence of approximating Solutions}

We will prove Theorem 4.4 by means of a four-level approximation, inspired by the construction of approximate solutions in $[13,10,12]$ for the study of the compressible Navier-Stokes equations and in [2] for the study of the incompressible FENE-P model. In this section we will describe our four-level approximation scheme. In subsequent sections we will prove the existence of solutions to each of the approximation levels, the convergence of the approximating solution sequence at each level, and hence we will complete the proof of Theorem 4.4.

6.1. Mollification of the initial data. First of all, we consider a mollification of the initial data by using the mollifier introduced in Section 5.1.

Let $\Omega \subset \mathbb{R}^{d}$ be a bounded $C^{2, \beta}$ domain, with $\beta \in(0,1)$, and let the initial data $\left(\varrho_{0}, \mathbf{u}_{0}, \mathbb{T}_{0}\right)$ be given, as in $(3.1)$. We consider the zero-extension of $\varrho_{0}$ and $\mathbf{u}_{0}$ to the whole of $\mathbb{R}^{d}$, still denoted by the same symbols, outside of the domain $\Omega$. We then define, for $\theta \in(0,1)$, the following mollified initial data for $\varrho_{0}$ and $\mathbf{u}_{0}$, and thanks to the properties of the 
classical Friedrichs mollifier listed in Lemma 5.1 and $(3.1)_{1,2}$, we have the following bounds and convergence results, as $\theta \rightarrow 0:$

$$
\varrho_{0, \theta}=\theta+S_{\theta}\left[\varrho_{0}\right] \in C^{\infty}\left(\mathbb{R}^{d}\right), \quad \theta \leq \varrho_{0, \theta} \leq C(\theta), \quad \varrho_{0, \theta} \rightarrow \varrho_{0} \quad \text { strongly in } L^{\gamma}(\Omega) ;
$$

(6.1) $\mathbf{u}_{0, \theta}=S_{\theta}\left[\mathbf{u}_{0}\right] \in C^{\infty}\left(\mathbb{R}^{d} ; \mathbb{R}^{d}\right), \quad \mathbf{u}_{0, \theta} \rightarrow \mathbf{u}_{0} \quad$ strongly in $L^{r}\left(\Omega ; \mathbb{R}^{d}\right), \quad \varrho_{0, \theta}\left|\mathbf{u}_{0, \theta}\right|^{2} \rightarrow \varrho_{0}\left|\mathbf{u}_{0}\right|^{2} \quad$ strongly in $L^{1}(\Omega) ;$ $\varrho_{0, \theta} \mathbf{u}_{0, \theta} \in C^{\infty}\left(\mathbb{R}^{d} ; \mathbb{R}^{d}\right), \quad \varrho_{0, \theta} \mathbf{u}_{0, \theta} \rightarrow \varrho_{0} \mathbf{u}_{0} \quad$ strongly in $L^{\frac{2 \gamma}{\gamma+1}}\left(\Omega ; \mathbb{R}^{d}\right) ;$

where $C(\theta)$ signifies a constant depending only on $\theta$, and $r$ is as defined in (3.1). It follows thanks to Sobolev embedding, as $\gamma>\frac{d}{2}$ by recalling (1.4), (5.1) and Lemma 5.1 that

$$
\left\|\varrho_{0, \theta}\right\|_{L^{\infty}\left(\mathbb{R}^{d}\right)} \leq \theta+\left\|S_{\theta}\left[\varrho_{0}\right]\right\|_{L^{\infty}\left(\mathbb{R}^{d}\right)} \leq \theta+C\left\|S_{\theta}\left[\varrho_{0}\right]\right\|_{W^{2, \gamma}\left(\mathbb{R}^{d}\right)} \leq \theta+C \theta^{-2}\left\|\varrho_{0}\right\|_{L^{\gamma}(\Omega)},
$$

and we can therefore take $C(\theta) \approx \theta^{-2}$, as $\theta \rightarrow 0$, in $(6.1)_{1}$.

The regularization of $\mathbb{T}_{0}$ is slightly different, as in Section 7.3 below we wish to apply Lemma 5.6 to a regularised version of (1.3) satisfying the boundary condition (1.10) and the regularised initial data. Given $\mathbb{T}_{0}$ satisfying $(3.1)_{3}$, for $\theta \in(0,1)$, we choose $T_{0, \theta}$ such that

$$
-\theta \Delta_{x} \mathbb{T}_{0, \theta}+\mathbb{T}_{0, \theta}=\mathbb{T}_{0} \quad \text { in } \Omega, \quad\left(\nu \cdot \nabla_{x}\right) \mathbb{T}_{0, \theta}=\mathbb{O} \quad \text { on } \partial \Omega .
$$

As $\Omega \subset \mathbb{R}^{d}$ is a bounded $C^{2, \beta}$ domain, with $\beta \in(0,1)$, it follows (cf. Theorem 2.4.2.7 in [16]) that the unique solution of (6.3) is such that

$$
\mathbb{T}_{0, \theta} \in W^{2, s}\left(\Omega ; \mathbb{R}^{d \times d}\right), \quad \text { for any } s \in[1, \infty) .
$$

Hence, $\mathbb{T}_{0, \theta}$, for any $\theta \in(0,1)$, satisfies the regularity requirement on the initial datum in the statement of Lemma 5.6 . In addition, it is easily deduced from (6.3) that

$$
\mathbb{T}_{0, \theta} \rightarrow \mathbb{T}_{0} \quad \text { strongly in } L^{2}\left(\Omega ; \mathbb{R}^{d \times d}\right), \quad \text { as } \theta \rightarrow 0 .
$$

Furthermore, on noting $(3.1)_{3}$ and adapting the maximum/minimum principle techniques in Lemma 5.2 in [1] and Lemma 6.2 in [2], we easily deduce that

$$
\mathbb{T}_{0, \theta}=\mathbb{T}_{0, \theta}^{\mathrm{T}}, \quad \operatorname{tr}\left(\mathbb{T}_{0, \theta}\right) \in\left[d \tau_{\min }, \mathfrak{b}^{\star}\right] \quad \text { and } \quad \tau_{\min } \mathbb{I} \leq \mathbb{T}_{0, \theta} \leq \mathfrak{b}^{\star} \mathbb{I}
$$

Moreover, it follows from (6.5), (6.6) and the Vitali convergence theorem, on possibly extracting a subsequence, that

$$
\mathbb{T}_{0, \theta} \rightarrow \mathbb{T}_{0} \quad \text { strongly in } L^{s}\left(\Omega ; \mathbb{R}^{d \times d}\right), \quad \text { for any } s \in[1, \infty), \quad \text { as } \theta \rightarrow 0 .
$$

Next, it follows from (1.8), (6.6) and (3.11) that

$$
\left\|\Pi\left(\mathbb{T}_{0, \theta}\right)\right\|_{L^{\infty}\left(\Omega ; \mathbb{R}^{d \times d}\right)} \leq C .
$$

Hence, (1.8), (6.8), (6.7) and the Vitali convergence theorem, by possibly extracting a subsequence, yields that for any $s \in[1, \infty):$

$$
\Pi\left(\mathbb{T}_{0, \theta}\right) \rightarrow \Pi\left(\mathbb{T}_{0}\right) \quad \text { strongly in } L^{s}\left(\Omega ; \mathbb{R}^{d \times d}\right), \quad \text { as } \theta \rightarrow 0 .
$$

Remark 6.1. We note that our conditions on $\mathbb{T}_{0},(3.1)_{3}$, are the same as those in $[2]$, which are stronger than those in [21] for the incompressible FENE-P model, where only $\mathbb{T}_{0}=\mathbb{T}_{0}^{\mathrm{T}}>0$ for a.e. $x \in \Omega$ with $\log \left(1-\frac{\operatorname{tr}\left(\mathbb{T}_{0}\right)}{\mathfrak{b}}\right) \in L^{1}\left(\Omega ; \mathbb{R}^{d \times d}\right)$ is assumed. In the compressible case we need to approximate $\mathbb{T}_{0}$ by $\mathbb{T}_{0, \theta}$ so that we can apply Lemma 5.6 to a regularised version of (1.3) satisfying the boundary condition (1.10) and the regularised initial data. This requires the construction stated above in this section. In addition, we need to prove (6.9), which is needed for $s=1$ in the proof of Lemma 11.1 below. We require our stronger assumptions on $\mathbb{T}_{0}$ in order to prove (6.9) even for $s=1$. Moreover, we note that in [21] no construction is given for the approximation of the initial data. It is just stated as an assumption at the start of Section 3 that an approximation satisfying the relevant properties exists.

6.2. First level: artificial pressure approximation. Let $\sigma_{1}>0$ be small and $\Gamma>\max \{\gamma, 8\}$. We consider the following system of equations for $\left(\varrho_{\sigma_{1}}, \mathbf{u}_{\sigma_{1}}, \mathbb{T}_{\sigma_{1}}\right)$, which results from a modification of the pressure in the system (1.1)-

$$
\begin{gathered}
\partial_{t} \varrho_{\sigma_{1}}+\operatorname{div}_{x}\left(\varrho_{\sigma_{1}} \mathbf{u}_{\sigma_{1}}\right)=0 \\
\partial_{t}\left(\varrho_{\sigma_{1}} \mathbf{u}_{\sigma_{1}}\right)+\operatorname{div}_{x}\left(\varrho_{\sigma_{1}} \mathbf{u}_{\sigma_{1}} \otimes \mathbf{u}_{\sigma_{1}}\right)+\nabla_{x} p\left(\varrho_{\sigma_{1}}\right)+\sigma_{1} \nabla_{x} \varrho_{\sigma_{1}}^{\Gamma}-\operatorname{div}_{x} \mathbb{S}\left(\nabla_{x} \mathbf{u}_{\sigma_{1}}\right) \\
=\operatorname{div}_{x}\left(\mathbb{A}\left(\mathbb{T}_{\sigma_{1}}\right)-\Pi\left(\mathbb{T} \sigma_{1}\right) \mathbb{I}\right)+\varrho_{\sigma_{1}} \mathbf{f} \\
\partial_{t} \mathbb{T}_{\sigma_{1}}+\left(\mathbf{u}_{\sigma_{1}} \cdot \nabla_{x}\right) \mathbb{T}_{\sigma_{1}}-\left(\nabla_{x} \mathbf{u}_{\sigma_{1}} \mathbb{T}_{\sigma_{1}}+\mathbb{T}_{\sigma_{1}} \nabla_{x}^{\mathrm{T}} \mathbf{u}_{\sigma_{1}}\right)=\varepsilon \Delta_{x} \mathbb{T}_{\sigma_{1}}-\frac{1}{2 \lambda} \mathbb{A}\left(\mathbb{T}_{\sigma_{1}}\right)
\end{gathered}
$$


We impose the same boundary conditions as in (1.9), (1.10) and we consider the mollified initial data defined in (6.1) and (6.3). Of course, the solution $\left(\varrho_{\sigma_{1}}, \mathbf{u}_{\sigma_{1}}, \mathbb{T}_{\sigma_{1}}\right)$ also depends on $\theta$ through the initial data $\left(\varrho_{0, \theta}, \mathbf{u}_{0, \theta}, \mathbb{T}_{0, \theta}\right)$, but we suppress this dependence in the notation $\left(\varrho_{\sigma_{1}}, \mathbf{u}_{\sigma_{1}}, \mathbb{T}_{\sigma_{1}}\right)$ here and below.

6.3. Second level: dissipative approximation. Let $\sigma_{2}>0$ be small. We consider the following system of equations for $\left(\varrho_{\sigma_{2}}, \mathbf{u}_{\sigma_{2}}, \mathbb{T}_{\sigma_{2}}\right)$, where a dissipative term is added to the continuity equation and, in order to maintain an energy bound, a term is added to the momentum equation:

$$
\begin{gathered}
\partial_{t}\left(\varrho_{\sigma_{2}} \mathbf{u}_{\sigma_{2}}\right)+\operatorname{div}_{x}\left(\varrho_{\sigma_{2}} \mathbf{u}_{\sigma_{2}} \otimes \mathbf{u}_{\sigma_{2}}\right)+\nabla_{x} p\left(\varrho_{\sigma_{2}}\right)+\frac{\sigma_{1} \nabla_{x} \varrho_{\sigma_{2}}^{\Gamma}-\operatorname{div}_{x} \mathbb{S}\left(\nabla_{x} \mathbf{u}_{\sigma_{2}}\right)}{=\operatorname{div}_{x}\left(\mathbb{A}\left(\mathbb{T}_{\sigma_{2}}\right)-\Pi\left(\mathbb{T}_{\sigma_{2}}\right) \mathbb{I}\right)+\varrho_{\sigma_{2}} \mathbf{f}-\sigma_{2} \nabla_{x} \mathbf{u}_{\sigma_{2}} \nabla_{x} \varrho_{\sigma_{2}},} \\
\partial_{t} \mathbb{T}_{\sigma_{2}}+\left(\mathbf{u}_{\sigma_{2}} \cdot \nabla_{x}\right) \mathbb{T}_{\sigma_{2}}-\left(\nabla_{x} \mathbf{u}_{\sigma_{2}} \mathbb{T}_{\sigma_{2}}+\mathbb{T}_{\sigma_{2}} \nabla_{x}^{\mathrm{T}} \mathbf{u}_{\sigma_{2}}\right)=\varepsilon \Delta_{x} \mathbb{T}_{\sigma_{2}}-\frac{1}{2 \lambda} \mathbb{A}\left(\mathbb{T}_{\sigma_{2}}\right) .
\end{gathered}
$$

We consider the mollified initial data defined in (6.1) and (6.3). Since the $\sigma_{2}$-regularised equation (6.13) is now parabolic, in addition to the boundary conditions stated in (1.9), (1.10) we shall also require that

$$
\nu \cdot \nabla_{x} \varrho_{\sigma_{2}}=0 \quad \text { on }(0, T] \times \partial \Omega .
$$

6.4. Third level: Galerkin approximation. By the classical theory of eigenvalue problems for symmetric linear elliptic operators (see, for example, Theorem 1 in Section 6.5 in [9]), one deduces the existence of a countably infinite sequence of eigenvalues $0<l_{1} \leq l_{2} \leq \cdots$ with $l_{n} \rightarrow \infty, n \rightarrow \infty$, and an associated orthogonal eigenfunction basis in $L^{2}\left(\Omega ; \mathbb{R}^{d}\right)$, denoted by $\left(\boldsymbol{\psi}_{n}\right)_{n \in \mathbb{N}}$, such that

$$
-\Delta_{x} \boldsymbol{\psi}_{n}=l_{n} \boldsymbol{\psi}_{n} \text { in } \Omega, \quad \boldsymbol{\psi}_{n}=\mathbf{0} \text { on } \partial \Omega
$$

Moreover, $\boldsymbol{\psi}_{n} \in W_{0}^{1,2}\left(\Omega ; \mathbb{R}^{d}\right) \cap W^{2,2}\left(\Omega ; \mathbb{R}^{d}\right) \cap C^{\infty}\left(\Omega ; \mathbb{R}^{d}\right)$ and $\boldsymbol{\psi}_{n} \in C^{2, \beta}\left(\bar{\Omega} ; \mathbb{R}^{d}\right)$ since $\Omega$ is a $C^{2, \beta}$ domain, with $\beta \in(0,1)$; by a classical Schauder type elliptic regularity estimate and Sobolev embedding, one also has that

$$
\left\|\boldsymbol{\psi}_{n}\right\|_{C^{2, \beta}\left(\bar{\Omega} ; \mathbb{R}^{d}\right)} \leq C\left(l_{n}\right)\left\|\boldsymbol{\psi}_{n}\right\|_{L^{2}\left(\Omega ; \mathbb{R}^{d}\right)}, \quad \text { with } C\left(l_{n}\right) \leq C l_{n}^{2}, \text { for } n=1,2, \ldots
$$

We define the $n$-dimensional Hilbert space $X_{n}$, with inner product $\langle\cdot, \cdot\rangle$, by

$$
X_{n}:=\operatorname{span}\left\{\boldsymbol{\psi}_{1}, \ldots, \boldsymbol{\psi}_{n}\right\}, \quad\langle\mathbf{v}, \mathbf{w}\rangle=\int_{\Omega} \mathbf{v} \cdot \mathbf{w} \mathrm{d} x, \quad \mathbf{v}, \mathbf{w} \in X_{n} .
$$

We denote by $P_{n}$ the orthogonal projection in $L^{2}\left(\Omega ; \mathbb{R}^{d}\right)$ onto the linear subspace $X_{n}$, and we consider the following problem:

$$
\begin{aligned}
& \mathbf{u}_{n} \in C\left([0, T], X_{n}\right), \mathbf{u}_{n}(0)=\mathbf{u}_{0, n}=P_{n} \mathbf{u}_{0, \theta} ; \quad \text { for any } \boldsymbol{\varphi}_{n} \in X_{n}: \\
& \begin{aligned}
\left.\int_{\Omega} \partial_{t}\left(\varrho_{n} \mathbf{u}_{n}\right) \cdot \boldsymbol{\varphi}_{n} \mathrm{~d} x-\int_{\Omega}\left[\varrho_{n} \mathbf{u}_{n} \otimes \mathbf{u}_{n}-\mathbb{S}\left(\nabla_{x} \mathbf{u}_{n}\right)\right]: \nabla_{x} \boldsymbol{\varphi}_{n} \mathrm{~d} x-\int_{\Omega}\left[p\left(\varrho_{n}\right)+\sigma_{1} \varrho_{n}^{\Gamma}\right]\right] \operatorname{div}_{x} \boldsymbol{\varphi}_{n} \mathrm{~d} x \\
\left.\quad=-\int_{\Omega}\left[\mathbb{A}\left(\mathbb{T}_{n}\right)-\Pi\left(\mathbb{T}_{n}\right) \mathbb{I}\right]: \nabla_{x} \boldsymbol{\varphi}_{n} \mathrm{~d} x+\int_{\Omega}\left[\varrho_{n} \mathbf{f}-\sigma_{2} \nabla_{x} \mathbf{u}_{n} \nabla_{x} \varrho_{n}\right]\right] \cdot \boldsymbol{\varphi}_{n} \mathrm{~d} x
\end{aligned}
\end{aligned}
$$

where $\mathbf{u}_{0, \theta}$ is the mollified initial datum for $\mathbf{u}_{0}$ defined in $(6.1)$, and $\varrho_{n}, \mathbb{T}_{n}$ are determined by the parabolic equations

$$
\begin{aligned}
\partial_{t} \varrho_{n}+\operatorname{div}_{x}\left(\varrho_{n} \mathbf{u}_{n}\right) & =\sigma_{2} \Delta_{x} \varrho_{n}, \\
\partial_{t} \mathbb{T}_{n}+\left(\mathbf{u}_{n} \cdot \nabla_{x}\right) \mathbb{T}_{n}-\left(\nabla_{x} \mathbf{u}_{n} \mathbb{T}_{n}+\mathbb{T}_{n} \nabla_{x}^{\mathrm{T}} \mathbf{u}_{n}\right) & =\varepsilon \Delta_{x} \mathbb{T}_{n}-\frac{1}{2 \lambda} \mathbb{A}\left(\mathbb{T}_{n}\right),
\end{aligned}
$$

subject to the boundary conditions stated in (1.9), (1.10) and (6.16), and the mollified initial data defined in (6.1) and (6.3) for $\varrho_{n}, \mathbf{u}_{n}$ and $\mathbb{T}_{n}$. 
6.5. Fourth level: regularisation of the conformation tensor. As pointed out in Section 3, the a priori bounds are obtained by assuming that $\mathbb{T}$ is symmetric positive definite with $\operatorname{tr}(\mathbb{T})<\mathfrak{b}$, which we do not have a priori. Thus, inspired by the work of Barrett \& Boyaval [2] for a finite element approximation of the incompressible FENE-P model, we will employ a regularisation for $\mathbb{T}$ to construct a family of symmetric positive definite approximations of $\mathbb{T}$, which satisfy bounds on their logarithm and inverse similar to the ones in Section 3. This involves introducing an approximation of $\operatorname{tr}(\mathbb{T})$ as a new unknown.

We introduce the following generalisation of scalar functions to symmetric matrix functions: let $g: \mathbb{R} \rightarrow \mathbb{R}$ be a scalar function and let $\mathbb{P} \in \mathbb{R}^{d \times d}$ be a real symmetric matrix; then, one has the following diagonalisation:

$$
\mathbb{P}=\mathbb{Q D} \mathbb{Q}^{\mathrm{T}}, \quad \mathbb{Q} \text { is an orthogonal matrix, } \mathbb{D}=\operatorname{diag}\left\{\mathfrak{l}_{1}, \ldots, \mathfrak{l}_{d}\right\},
$$

where $\mathfrak{l}_{j}, j=1, \ldots, d$, are the eigenvalues of $\mathbb{P}$. We define $g(\mathbb{P})$ and $g^{\prime}(\mathbb{P})$ by the following formulae:

$$
g(\mathbb{P})=\mathbb{Q}(g(\mathbb{D})) \mathbb{Q}^{\mathrm{T}}, \quad g^{\prime}(\mathbb{P})=\mathbb{Q}\left(g^{\prime}(\mathbb{D})\right) \mathbb{Q}^{\mathrm{T}},
$$

where

$$
g(\mathbb{D}):=\operatorname{diag}\left\{g\left(\mathfrak{l}_{1}\right), \ldots, g\left(\mathfrak{l}_{d}\right)\right\}, \quad g^{\prime}(\mathbb{D}):=\operatorname{diag}\left\{g^{\prime}\left(\mathfrak{l}_{1}\right), \ldots, g^{\prime}\left(\mathfrak{l}_{d}\right)\right\} .
$$

With these definitions, we have the following lemma.

Lemma 6.2. Let $g \in C^{1, \beta}(\mathbb{R})$, with $\beta \in(0,1]$.

(i) Let $\mathbb{P} \in W^{1,2}\left(0, T ; \mathbb{R}^{d \times d}\right)$ be symmetric. Then, the matrix function $t \in(0, T) \mapsto g(\mathbb{P}(t)) \in \mathbb{R}^{d \times d}$, defined by $(6.22)$, is differentiable a.e. on $(0, T)$ and satisfies the identity

$$
\partial_{t} \operatorname{tr}(g(\mathbb{P}))=\operatorname{tr}\left(g^{\prime}(\mathbb{P}) \partial_{t} \mathbb{P}\right)=\partial_{t} \mathbb{P}: g^{\prime}(\mathbb{P}) \quad \text { a.e. on }(0, T) .
$$

(ii) Let $\mathbb{P} \in W^{1,2}\left(\Omega ; \mathbb{R}^{d \times d}\right)$ be symmetric. Then, the matrix function $x \in \Omega \mapsto g(\mathbb{P}(x)) \in \mathbb{R}^{d \times d}$, defined by (6.22), is weakly differentiable on $\Omega$ and satisfies the identity

$$
\partial_{x_{i}} \operatorname{tr}(g(\mathbb{P}))=\operatorname{tr}\left(g^{\prime}(\mathbb{P}) \partial_{x_{i}} \mathbb{P}\right)=\partial_{x_{i}} \mathbb{P}: g^{\prime}(\mathbb{P}), \quad i=1, \ldots, d, \quad \text { a.e. on } \Omega .
$$

Proof. Part (i) of this lemma is stated as Lemma 6.1, and proved in Appendix B, of [3] in the case of $g$ being concave or convex. However, the proof there does not generalise to part (ii). So here we give an alternative proof of part (i), which generalises immediately to part (ii), and, in addition, does not require $g$ to be convex or concave.

By a density argument, we can approximate $\mathbb{P} \in W^{1,2}\left(0, T ; \mathbb{R}^{d \times d}\right)$ by $\mathbb{P}_{\delta} \in C^{\infty}\left([0, T] ; \mathbb{R}^{d \times d}\right)$ such that

$$
\mathbb{P}_{\delta} \rightarrow \mathbb{P} \quad \text { strongly in } W^{1,2}\left(0, T ; \mathbb{R}^{d \times d}\right), \quad \text { as } \delta \rightarrow 0 .
$$

Clearly, (6.24) holds for $\mathbb{P}$ replaced by $\mathbb{P}_{\delta}$ for all $t \in[0, T]$, which yields that

$$
-\int_{0}^{T} \operatorname{tr}\left(g\left(\mathbb{P}_{\delta}\right)\right) \partial_{t} \eta \mathrm{d} t=\int_{0}^{T} \partial_{t} \mathbb{P}_{\delta}: g^{\prime}\left(\mathbb{P}_{\delta}\right) \eta \mathrm{d} t \quad \forall \eta \in C_{0}^{\infty}(0, T) .
$$

As $g \in C^{1, \beta}(\mathbb{R})$, with $\beta \in(0,1]$, it follows from Theorem 1.1 in $[27]$ that

$$
\left\|\operatorname{tr}\left(g(\mathbb{P})-g\left(\mathbb{P}_{\delta}\right)\right)\right\|_{L^{1}(0, T)} \leq C\left\|\mathbb{P}-\mathbb{P}_{\delta}\right\|_{L^{1}(0, T)} \quad \text { and } \quad\left\|g^{\prime}(\mathbb{P})-g^{\prime}\left(\mathbb{P}_{\delta}\right)\right\|_{L^{2}(0, T)} \leq C\left\|\mathbb{P}-\mathbb{P}_{\delta}\right\|_{L^{2}(0, T)}^{\beta} .
$$

Hence, by noting (6.28) and (6.26), we can pass to the limit $\delta \rightarrow 0$ in (6.27) to obtain

$$
-\int_{0}^{T} \operatorname{tr}(g(\mathbb{P})) \partial_{t} \eta \mathrm{d} t=\int_{0}^{T} \partial_{t} \mathbb{P}: g^{\prime}(\mathbb{P}) \eta \mathrm{d} t \quad \forall \eta \in C_{0}^{\infty}(0, T),
$$

and therefore the desired result (6.24).

This proof of part (i) is easily adapted to prove part (ii).

Next, let $\sigma_{3}>0$ be small, in the sense that $\sigma_{3}<\theta<1$, and for fixed $L \geq \mathfrak{b}$, we define

$$
\chi_{\sigma_{3}}(s):=\left\{\begin{array}{cl}
\sigma_{3}^{-1} & \text { if } s \geq \sigma_{3}^{-1}, \\
s & \text { if } s \in\left[\sigma_{3}, \sigma_{3}^{-1}\right], \\
\sigma_{3} & \text { if } s \leq \sigma_{3}
\end{array} \quad \text { and } \quad \chi_{\sigma_{3}}^{L}(s):=\left\{\begin{array}{cl}
L & \text { if } s \geq L, \\
s & \text { if } s \in\left[\sigma_{3}, L\right], \\
\sigma_{3} & \text { if } s \leq \sigma_{3} .
\end{array}\right.\right.
$$

Similarly to [1], we introduce the concave logarithmic cut-off function $\mathcal{G}_{\sigma_{3}}: \mathbb{R} \rightarrow \mathbb{R}$, defined by

$$
\mathcal{G}_{\sigma_{3}}(s):=\left\{\begin{array}{cl}
\sigma_{3} s-\log \sigma_{3}-1 & \text { if } s \geq \sigma_{3}^{-1} \\
\log s & \text { if } s \in\left[\sigma_{3}, \sigma_{3}^{-1}\right], \\
\sigma_{3}^{-1} s+\log \sigma_{3}-1 & \text { if } s \leq \sigma_{3}
\end{array}\right.
$$

Since $\mathcal{G}_{\sigma_{3}}^{\prime}(s)=\left[\chi_{\sigma_{3}}(s)\right]^{-1}$ for all $s \in \mathbb{R}$, we have, for any real symmetric matrix $\mathbb{P}$, that

$$
\mathcal{G}_{\sigma_{3}}^{\prime}(\mathbb{P})=\left[\chi_{\sigma_{3}}(\mathbb{P})\right]^{-1} .
$$


Similarly to [2], in order to define our fourth level of approximation, we introduce another unknown, $z_{n, \sigma_{3}}$, which approximates $\operatorname{tr}\left(\mathbb{T}_{n, \sigma_{3}}\right)$. We state the reason for this in Remark 7.2 below. Our fourth level of approximation is as follows:

$(6.33)$

$$
\begin{aligned}
& \mathbf{u}_{n, \sigma_{3}} \in C\left(\left[0, T_{n}\right], X_{n}\right), \mathbf{u}_{n, \sigma_{3}}(0)=\mathbf{u}_{0, n}=P_{n} \mathbf{u}_{0, \theta} ; \quad \text { for any } \boldsymbol{\varphi}_{n} \in X_{n}: \\
& \int_{\Omega} \partial_{t}\left(\varrho_{n, \sigma_{3}} \mathbf{u}_{n, \sigma_{3}}\right) \cdot \boldsymbol{\varphi}_{n} \mathrm{~d} x-\int_{\Omega}\left[\varrho_{n, \sigma_{3}} \mathbf{u}_{n, \sigma_{3}} \otimes \mathbf{u}_{n, \sigma_{3}}-\mathbb{S}\left(\nabla_{x} \mathbf{u}_{n, \sigma_{3}}\right)\right]: \nabla_{x} \boldsymbol{\varphi}_{n} \mathrm{~d} x-\int_{\Omega}\left[p\left(\varrho_{n, \sigma_{3}}\right)+\sigma_{1} \varrho_{n, \sigma_{3}}^{\Gamma}\right] \operatorname{div}_{x} \boldsymbol{\varphi}_{n} \mathrm{~d} x \\
&=-\int_{\Omega}\left[\left[\left(\left[\chi_{\sigma_{3}}\left(1-\frac{z_{n, \sigma_{3}}}{\mathfrak{b}}\right)\right]^{-1} \chi_{\sigma_{3}}\left(\mathbb{T}_{n, \sigma_{3}}^{S}\right)-\Pi_{\sigma_{3}}^{(1)}\left(\mathbb{T}_{n, \sigma_{3}}^{S}, z_{n, \sigma_{3}}\right) \mathbb{I}\right)\right]\right]: \nabla_{x} \boldsymbol{\varphi}_{n} \mathrm{~d} x \\
& \quad+\int_{\Omega}\left[\varrho_{n, \sigma_{3}} \mathbf{f}-\sigma_{2} \nabla_{x} \mathbf{u}_{n, \sigma_{3}} \nabla_{x} \varrho_{n, \sigma_{3}}\right] \cdot \boldsymbol{\varphi}_{n} \mathrm{~d} x,
\end{aligned}
$$

where $\mathbf{u}_{0, \theta}$ is the mollified initial datum for $\mathbf{u}_{0}$ defined in (6.1), and subject to the boundary condition (1.9) for $\mathbf{u}_{n, \sigma_{3}}$. Here

$$
\mathbb{T}_{n, \sigma_{3}}^{S}:=\frac{1}{2}\left(\mathbb{T}_{n, \sigma_{3}}+\left(\mathbb{T}_{n, \sigma_{3}}\right)^{\mathrm{T}}\right),
$$

$z_{n, \sigma_{3}}$ is an approximation of $\operatorname{tr}\left(\mathbb{T}_{n, \sigma_{3}}^{S}\right)$ and $\Pi_{\sigma_{3}}^{(1)}$ is a regularisation of $\Pi,(1.8)$, based on $\chi_{\sigma_{3}},(6.30)$ :

$$
\begin{aligned}
\Pi_{\sigma_{3}}^{(1)}\left(\mathbb{T}_{n, \sigma_{3}}^{S}, z_{n, \sigma_{3}}\right)=\frac{1}{2}\left[\mathfrak{a} \operatorname{tr}\left(\log \left(\chi_{\sigma_{3}}\left(\mathbb{T}_{n, \sigma_{3}}^{S}\right)\right)\right)+\mathfrak{b} \log \chi_{\sigma_{3}}\left(1-\frac{z_{n, \sigma_{3}}}{\mathfrak{b}}\right)\right] \\
+\frac{\mathfrak{b}}{2}\left[\chi_{\sigma_{3}}\left(1-\frac{z_{n, \sigma_{3}}}{\mathfrak{b}}\right)\right]^{-1}\left(1-\frac{\operatorname{tr}\left(\chi_{\sigma_{3}}\left(\mathbb{T}_{n, \sigma_{3}}^{S}\right)\right)}{\mathfrak{b}}\right) .
\end{aligned}
$$

Hence, the choice of the arbitrary constant in (1.8). In addition, $\varrho_{n, \sigma_{3}}, \mathbb{T}_{n, \sigma_{3}}$ and $z_{n, \sigma_{3}}$ are determined by the parabolic equations

$$
\begin{aligned}
& \partial_{t} \varrho_{n, \sigma_{3}}+\operatorname{div}_{x}\left(\varrho_{n, \sigma_{3}} \mathbf{u}_{n, \sigma_{3}}\right)=\sigma_{2} \Delta_{x} \varrho_{n, \sigma_{3}} \\
& \partial_{t} \mathbb{T}_{n, \sigma_{3}}-\varepsilon \Delta_{x} \mathbb{T}_{n, \sigma_{3}}=\mathbf{F}_{n, \sigma_{3}}:=-\left(\mathbf{u}_{n, \sigma_{3}} \cdot \nabla_{x}\right) \chi_{\sigma_{3}}\left(\mathbb{T}_{n, \sigma_{3}}^{S}\right)-\frac{1}{2 \lambda} \mathbb{A}_{n, \sigma_{3}}^{L} \\
& +\left(\nabla_{x} \mathbf{u}_{n, \sigma_{3}} \chi_{\sigma_{3}}\left(\mathbb{T}_{n, \sigma_{3}}^{S}\right)+\chi_{\sigma_{3}}\left(\mathbb{T}_{n, \sigma_{3}}^{S}\right) \nabla_{x}^{\mathrm{T}} \mathbf{u}_{n, \sigma_{3}}\right), \\
& \partial_{t} z_{n, \sigma_{3}}-\varepsilon \Delta_{x} z_{n, \sigma_{3}}=F_{z, n, \sigma_{3}}:=\mathfrak{b}\left(\mathbf{u}_{n, \sigma_{3}} \cdot \nabla_{x}\right) \chi_{\sigma_{3}}\left(1-\frac{z_{n, \sigma_{3}}}{\mathfrak{b}}\right)-\frac{1}{2 \lambda} \operatorname{tr}\left(\mathbb{A}_{n, \sigma_{3}}^{L}\right)+2 \operatorname{tr}\left(\nabla_{x} \mathbf{u}_{n, \sigma_{3}} \chi_{\sigma_{3}}\left(\mathbb{T}_{n, \sigma_{3}}^{S}\right)\right) \\
& +\mathfrak{b} \operatorname{div}_{x} \mathbf{u}_{n, \sigma_{3}}\left[\chi_{\sigma_{3}}\left(1-\frac{z_{n, \sigma_{3}}}{\mathfrak{b}}\right)-\left(1-\frac{\operatorname{tr}\left(\chi_{\sigma_{3}}\left(\mathbb{T}_{n, \sigma_{3}}^{S}\right)\right)}{\mathfrak{b}}\right)\right],
\end{aligned}
$$

where

$$
\mathbb{A}_{n, \sigma_{3}}^{L}:=\left(\left[\chi_{\sigma_{3}}\left(1-\frac{z_{n, \sigma_{3}}}{\mathfrak{b}}\right)\right]^{-1} \mathbb{I}-\mathfrak{a}\left[\chi_{\sigma_{3}}\left(\mathbb{T}_{n, \sigma_{3}}^{S}\right)\right]^{-1}\right) \chi_{\sigma_{3}}^{L}\left(\mathbb{T}_{n, \sigma_{3}}^{S}\right) .
$$

The equations (6.36), (6.37) will be considered subject to the boundary conditions in (6.16), (1.10) and the initial data defined in (6.1) and (6.3) for $\varrho_{n, \sigma_{3}}, \mathbb{T}_{n, \sigma_{3}}$. The equation (6.38) will be considered subject to the initial and boundary conditions

$$
z_{n, \sigma_{3}}(0, \cdot)=\operatorname{tr}\left(\mathbb{T}_{0, \theta}(\cdot)\right) \quad \text { and } \quad \nu \cdot \nabla_{x} z_{n, \sigma_{3}}=0 \quad \text { on }(0, T] \times \partial \Omega .
$$

Although $\varrho_{n, \sigma_{3}}, \mathbf{u}_{n, \sigma_{3}}, \mathbb{T}_{n, \sigma_{3}}$ and $z_{n, \sigma_{3}}$ depend on $L$, via $\mathbb{A}_{n, \sigma_{3}}^{L}$, (6.39), we suppress this dependence in the notation as $L \geq \mathfrak{b}$ is fixed.

It follows from (6.37) and (6.38) that

$$
\begin{aligned}
\partial_{t}\left(z_{n, \sigma_{3}}-\operatorname{tr}\left(\mathbb{T}_{n, \sigma_{3}}\right)\right)- & \varepsilon \Delta_{x}\left(z_{n, \sigma_{3}}-\operatorname{tr}\left(\mathbb{T}_{n, \sigma_{3}}\right)\right)=F_{z, n, \sigma_{3}}-\operatorname{tr}\left(\mathbf{F}_{n, \sigma_{3}}\right) \\
& =\mathfrak{b} \operatorname{div}_{x}\left(\mathbf{u}_{n, \sigma_{3}}\left[\chi_{\sigma_{3}}\left(1-\frac{z_{n, \sigma_{3}}}{\mathfrak{b}}\right)-\left(1-\frac{\operatorname{tr}\left(\chi_{\sigma_{3}}\left(\mathbb{T}_{n, \sigma_{3}}^{S}\right)\right)}{\mathfrak{b}}\right)\right]\right) .
\end{aligned}
$$

Integrating (6.41) over $\Omega$, applying the initial and boundary conditions for (6.40), and the boundary conditions (1.9), (1.10) for $\mathbf{u}_{n, \sigma_{3}}$ and $\mathbb{T}_{n, \sigma_{3}}$ we obtain that

$$
\int_{\Omega}\left(z_{n, \sigma_{3}}(t, x)-\operatorname{tr}\left(\mathbb{T}_{n, \sigma_{3}}(t, x)\right)\right) \mathrm{d} x=0 \quad \text { for a.e. } t \in(0, T]
$$


provided solutions exist, which is discussed in the next section.

Finally, for later purposes, we also introduce an alternative regularisation of $\Pi,(1.8)$, to (6.35) based on $\mathcal{G}_{\sigma_{3}},(6.31)$ :

$$
\Pi_{\sigma_{3}}^{(2)}\left(\mathbb{T}_{n, \sigma_{3}}^{S}, z_{n, \sigma_{3}}\right)=\frac{1}{2}\left[\mathfrak{a} \operatorname{tr}\left(\mathcal{G}_{\sigma_{3}}\left(\mathbb{T}_{n, \sigma_{3}}^{S}\right)\right)+\mathfrak{b} \mathcal{G}_{\sigma_{3}}\left(1-\frac{z_{n, \sigma_{3}}}{\mathfrak{b}}\right)+\mathfrak{b}\right]
$$

\section{The FOURTH LEVEL OF APPROXIMATION}

For any $\sigma_{3}<\theta<1$ and any fixed $L \geq \mathfrak{b}$, by recalling (6.30), and any $n \in \mathbb{N}$, the problem (6.33) is a system of ordinary differential equations in $\mathbf{u}_{n, \sigma_{3}}$ with respect to $t$ because $X_{n}$ is a finite-dimensional space; the equations (6.36), (6.37), (6.38) are of parabolic type and are well-posed given any smooth $\mathbf{u}_{n, \sigma_{3}}$. The latter easily follows, as for example the boxed nonlinearities in (6.37) are globally Lipschitz. Here we note the Lipschitz continuity of $\chi_{\sigma_{3}}$ and $\chi_{\sigma_{3}}^{L}$, defined over the space of real symmetric matrices, follow from the Lipschitz continuity of $\chi_{\sigma_{3}}$ and $\chi_{\sigma_{3}}^{L}$ considered as a mapping from $\mathbb{R}$ into $\mathbb{R}$ (cf. Theorem 1.1 in [27]). Thus, locally in time, over a time interval $\left[0, T_{n, \sigma_{3}}\right]$, for some $T_{n, \sigma_{3}}>0$, the existence of a unique solution, denoted by $\left(\varrho_{n, \sigma_{3}}, \mathbf{u}_{n, \sigma_{3}}, \mathbb{T}_{n, \sigma_{3}}, z_{n, \sigma_{3}}\right)$, to the problem at the fourth level of approximation, posed in Section 6.5, is classical, see [20,13, 11, 22].

Since $\mathbf{u}_{n, \sigma_{3}} \in C\left(\left[0, T_{n, \sigma_{3}}\right], X_{n}\right)$, by the definition of $X_{n}$ in Section 6.4, we have

$$
\mathbf{u}_{n, \sigma_{3}} \in C\left(\left[0, T_{n, \sigma_{3}}\right], C^{2, \beta}\left(\bar{\Omega} ; \mathbb{R}^{d}\right)\right), \quad\left\|\mathbf{u}_{n, \sigma_{3}}(t)\right\|_{C^{2, \beta}\left(\bar{\Omega} ; \mathbb{R}^{d}\right)} \leq C(n)\left\|\mathbf{u}_{n, \sigma_{3}}(t)\right\|_{L^{2}\left(\Omega ; \mathbb{R}^{d}\right)} \text { for all } t \in\left[0, T_{n, \sigma_{3}}\right] .
$$

By similar arguments as in Section 2.1 in [13] concerning well-posedness and uniform bounds for parabolic equations, we have, for all $t \in\left(0, T_{n, \sigma_{3}}\right]$, that

$$
\begin{aligned}
& \left(\varrho_{n, \sigma_{3}}, \mathbb{T}_{n, \sigma_{3}}, z_{n, \sigma_{3}}\right) \in C\left(\left[0, T_{n, \sigma_{3}}\right] ; W^{1,2}(\Omega) \times W^{1,2}\left(\Omega ; \mathbb{R}^{d \times d}\right) \times W^{1,2}(\Omega)\right), \\
& \left(\varrho_{n, \sigma_{3}}, \mathbb{T}_{n, \sigma_{3}}, z_{n, \sigma_{3}}\right) \in L^{2}\left(0, T_{n, \sigma_{3}} ; W^{2,2}(\Omega) \times W^{2,2}\left(\Omega ; \mathbb{R}^{d \times d}\right) \times W^{2,2}(\Omega)\right), \quad \mathbb{T}_{n, \sigma_{3}} \text { is symmetric, } \\
& \theta \exp \left(-\int_{0}^{t}\left\|\operatorname{div}_{x} \mathbf{u}_{n, \sigma_{3}}\left(t^{\prime}\right)\right\|_{L^{\infty}(\Omega)} \mathrm{d} t^{\prime}\right) \leq \varrho_{n, \sigma_{3}}(t, x) \leq C(\theta) \exp \left(\int_{0}^{t}\left\|\operatorname{div}_{x} \mathbf{u}_{n, \sigma_{3}}\left(t^{\prime}\right)\right\|_{L^{\infty}(\Omega)} \mathrm{d} t^{\prime}\right), \quad \text { for all } x \in \Omega, \\
& \left\|\varrho_{n, \sigma_{3}}(t)\right\|_{W^{1,2}(\Omega)}^{2}+\int_{0}^{t}\left\|\varrho_{n, \sigma_{3}}\left(t^{\prime}\right)\right\|_{W^{2,2}(\Omega)}^{2} \mathrm{~d} t^{\prime} \leq C\left(t, \theta, \sigma_{2},\left\|\nabla_{x} \mathbf{u}_{n, \sigma_{3}}\right\|_{L^{\infty}\left(\left(0, T_{n, \sigma_{3}}\right) \times \Omega ; \mathbb{R}^{d \times d}\right)}\right), \\
& \left\|\mathbb{T}_{n, \sigma_{3}}(t)\right\|_{W^{1,2}\left(\Omega ; \mathbb{R}^{d \times d}\right)}^{2}+\int_{0}^{t}\left\|\mathbb{T}_{n, \sigma_{3}}\left(t^{\prime}\right)\right\|_{W^{2,2}\left(\Omega ; \mathbb{R}^{d \times d}\right)}^{2} \mathrm{~d} t^{\prime} \leq C\left(t, \alpha, \theta, \sigma_{3}, L,\left\|\nabla_{x} \mathbf{u}_{n, \sigma_{3}}\right\|_{L^{\infty}\left(\left(0, T_{n, \sigma_{3}}\right) \times \Omega ; \mathbb{R}^{d \times d}\right)}\right), \\
& \left\|z_{n, \sigma_{3}}(t)\right\|_{W^{1,2}(\Omega)}^{2}+\int_{0}^{t}\left\|z_{n, \sigma_{3}}\left(t^{\prime}\right)\right\|_{W^{2,2}(\Omega)}^{2} \mathrm{~d} t^{\prime} \leq C\left(t, \alpha, \theta, \sigma_{3}, L,\left\|\nabla_{x} \mathbf{u}_{n, \sigma_{3}}\right\|_{L^{\infty}\left(\left(0, T_{n, \sigma_{3}}\right) \times \Omega ; \mathbb{R}^{d \times d}\right)}\right)
\end{aligned}
$$

The symmetry of $\mathbb{T}_{n, \sigma_{3}}$ can be deduced by using the symmetry of equation (6.37), the symmetry of $\mathbb{T}_{n, \sigma_{3}}^{S}$, the symmetry of the initial datum $\mathbb{T}_{n, \sigma_{3}}(0)=\mathbb{T}_{0, \theta} \geq \tau_{\min } \mathbb{I}$, the symmetry of the $\mathbb{T}_{n, \sigma_{3}}$ terms appearing on the right-hand side of (6.33), and the uniqueness of the solutions to equations (6.37), (6.38). The bounds on $\mathbb{T}_{n, \sigma_{3}}, z_{n, \sigma_{3}}$ in (7.2) can be derived similarly to those on the scalar function $\varrho_{n, \sigma_{3}}$, by observing, for example, that,

$$
\left|\chi_{\sigma_{3}}(\mathbb{P})\right| \leq \sigma_{3}^{-1}, \quad\left|\chi_{\sigma_{3}}^{L}(\mathbb{P})\right| \leq L \quad \text { for all symmetric } \mathbb{P} \in \mathbb{R}^{d \times d} .
$$

In the rest of this section we shall derive uniform bounds on the solution sequence, which guarantee that the existence time $T_{n . \sigma_{3}}$ identified above can be extended to $T$.

7.1. Some uniform bounds. We shall now develop some bounds that are uniform in $\sigma_{3}$ in the limit of $\sigma_{3} \rightarrow 0$. First, we require the following generalisation of Lemma 3.1.

Lemma 7.1. For $\sigma_{3}>0$, suppose that $\mathbb{P} \in C\left([0, T] ; W^{1,2}\left(\Omega ; \mathbb{R}^{m \times m}\right)\right), m \in \mathbb{N}$, is a symmetric matrix function, with $\Delta_{x} \mathbb{P} \in L^{2}\left(0, T ; L^{2}\left(\Omega ; \mathbb{R}^{m \times m}\right)\right)$, satisfying $\left(\nu \cdot \nabla_{x}\right) \mathbb{P}=\mathbb{O}$ on $(0, T] \times \partial \Omega ;$ then, $\left[\chi_{\sigma_{3}}(\mathbb{P})\right]^{-1} \in L^{\infty}\left(0, T ; W^{1,2}\left(\Omega ; \mathbb{R}^{m \times m}\right)\right)$, and

$$
\int_{\Omega} \Delta_{x} \mathbb{P}:\left[\chi_{\sigma_{3}}(\mathbb{P})\right]^{-1} \mathrm{~d} x=-\int_{\Omega} \nabla_{x} \mathbb{P}:: \nabla_{x}\left[\chi_{\sigma_{3}}(\mathbb{P})\right]^{-1} \mathrm{~d} x \geq \frac{1}{m} \int_{\Omega}\left|\nabla_{x} \operatorname{tr}\left(\log \chi_{\sigma_{3}}(\mathbb{P})\right)\right|^{2} \mathrm{~d} x, \quad \text { a.e. on }(0, T] .
$$

Proof. This lemma is stated as Lemma 7.1, and proved in Appendix C, of [3] in the case $m=d$ and for $\chi_{\sigma_{3}}(s):=$ $\max \left\{\sigma_{3}, s\right\}, s \in \mathbb{R}$. The proof given there immediately generalises to $m \in \mathbb{N}$ and the definition of $\chi_{\sigma_{3}}$ in (6.30).

Next, we note from (3.3) and (6.36) that

$$
\begin{aligned}
\frac{1}{2} \int_{\Omega}\left(\partial_{t} \varrho_{n, \sigma_{3}}\right)\left|\mathbf{u}_{n, \sigma_{3}}\right|^{2} \mathrm{~d} x+\int_{\Omega} \operatorname{div}_{x}\left(\varrho_{n, \sigma_{3}} \mathbf{u}_{n, \sigma_{3}} \otimes \mathbf{u}_{n, \sigma_{3}}\right) \cdot \mathbf{u}_{n, \sigma_{3}} \mathrm{~d} x \\
=\frac{1}{2} \int_{\Omega}\left(\partial_{t} \varrho_{n, \sigma_{3}}+\operatorname{div}_{x}\left(\varrho_{n, \sigma_{3}} \mathbf{u}_{n, \sigma_{3}}\right)\right)\left|\mathbf{u}_{n, \sigma_{3}}\right|^{2} \mathrm{~d} x \\
=\frac{\sigma_{2}}{2} \int_{\Omega} \Delta_{x} \varrho_{n, \sigma_{3}}\left|\mathbf{u}_{n, \sigma_{3}}\right|^{2} \mathrm{~d} x=-\sigma_{2} \int_{\Omega}\left(\nabla_{x} \mathbf{u}_{n, \sigma_{3}} \nabla_{x} \varrho_{n, \sigma_{3}}\right) \cdot \mathbf{u}_{n, \sigma_{3}} \mathrm{~d} x
\end{aligned}
$$


Similarly to the a priori bound (3.7), we deduce by taking $\varphi_{n}=\mathbf{u}_{n, \sigma_{3}}$ in (6.33), combining with (7.5) and the equation (6.36) tested with $b_{1}^{\prime}\left(\varrho_{n, \sigma_{3}}\right)$, where $b_{1}(r)=\frac{a}{\gamma-1} r^{\gamma}+\frac{\sigma_{1}}{\Gamma-1} r^{\Gamma}$, and noting the boundary condition (6.16) for $\varrho_{n, \sigma_{3}}$ that

$$
\begin{aligned}
\frac{\mathrm{d}}{\mathrm{d} t} \int_{\Omega} & {\left[\frac{1}{2} \varrho_{n, \sigma_{3}}\left|\mathbf{u}_{n, \sigma_{3}}\right|^{2}+\frac{a}{\gamma-1} \varrho_{n, \sigma_{3}}^{\gamma}+\frac{\sigma_{1}}{\Gamma-1} \varrho_{n, \sigma_{3}}^{\Gamma}\right] \mathrm{d} x+4 \sigma_{2} \int_{\Omega}\left[\frac{a}{\gamma}\left|\nabla_{x} \varrho_{n, \sigma_{3}}^{\frac{\gamma}{2}}\right|^{2}+\frac{\sigma_{1}}{\Gamma}\left|\nabla_{x} \varrho_{n, \sigma_{3}}^{\frac{\Gamma}{2}}\right|^{2}\right] \mathrm{d} x } \\
& +\int_{\Omega}\left[\mu^{S}\left|\frac{\nabla_{x} \mathbf{u}_{n, \sigma_{3}}+\nabla_{x}^{\mathrm{T}} \mathbf{u}_{n, \sigma_{3}}}{2}-\frac{1}{d}\left(\operatorname{div}_{x} \mathbf{u}_{n, \sigma_{3}}\right) \mathbb{I}\right|^{2}+\mu^{B}\left|\operatorname{div}_{x} \mathbf{u}_{n, \sigma_{3}}\right|^{2}\right] \mathrm{d} x \\
= & -\int_{\Omega}\left(\left[\chi_{\sigma_{3}}\left(1-\frac{z_{n, \sigma_{3}}}{\mathfrak{b}}\right)\right]^{-1} \chi_{\sigma_{3}}\left(\mathbb{T}_{n, \sigma_{3}}\right)-\Pi_{\sigma_{3}}^{(1)}\left(\mathbb{T}_{n, \sigma_{3}}, z_{n, \sigma_{3}}\right) \mathbb{I}\right): \nabla_{x} \mathbf{u}_{n, \sigma_{3}} \mathrm{~d} x+\int_{\Omega} \varrho_{n, \sigma_{3}} \mathbf{f} \cdot \mathbf{u}_{n, \sigma_{3}} \mathrm{~d} x
\end{aligned}
$$

for a.e. $t \in\left(0, T_{n, \sigma_{3}}\right]$, where we have used that $\mathbb{T}_{n, \sigma_{3}}^{S}=\mathbb{T}_{n, \sigma_{3}}$ (cf. the paragraph following eq. (7.2)). In addition, we note that in deriving (7.6) the right-hand side of (7.5) cancels with the term involving $\sigma_{2}$ on the right-hand side of (6.33).

To mimic the testing procedure of (1.3) with (3.8), we now take the inner product of (6.37) with $-\mathfrak{a} \mathcal{G}_{\sigma_{3}}^{\prime}\left(\mathbb{T}_{n, \sigma_{3}}\right)$ and (6.38) with $\mathcal{G}_{\sigma_{3}}^{\prime}\left(1-\frac{z_{n, \sigma_{3}}}{\mathfrak{b}}\right)$, respectively, where we recall (6.32). Integrating over $\Omega$, performing partial integration and noting the boundary conditions (1.9), (1.10) for $\mathbf{u}_{n, \sigma_{3}}, \mathbb{T}_{n, \sigma_{3}}$ and (6.40) for $z_{n, \sigma_{3}}$ we perform the analogue of (3.14)(3.18). It follows from $(7.2)_{5,6}$ that $\mathbb{T}_{n, \sigma_{3}} \in W^{1,2}\left(0, T_{n, \sigma_{3}} ; L^{2}\left(\Omega, \mathbb{R}^{d \times d}\right)\right)$ and $z_{n, \sigma_{3}} \in W^{1,2}\left(0, T_{n, \sigma_{3}} ; L^{2}(\Omega)\right)$. Hence, by Lemma 6.2, we have, as $\mathbb{T}_{n, \sigma_{3}}^{S}=\mathbb{T}_{n, \sigma_{3}}$ and $\mathcal{G}_{\sigma_{3}} \in C^{1,1}(\mathbb{R})$ is concave, that

$$
\begin{aligned}
\int_{\Omega} \partial_{t} z_{n, \sigma_{3}} \mathcal{G}_{\sigma_{3}}^{\prime}(1 & \left.-\frac{z_{n, \sigma_{3}}}{\mathfrak{b}}\right) \mathrm{d} x-\mathfrak{a} \int_{\Omega} \partial_{t} \mathbb{T}_{n, \sigma_{3}}: \mathcal{G}_{\sigma_{3}}^{\prime}\left(\mathbb{T}_{n, \sigma_{3}}\right) \mathrm{d} x \\
& =-\mathfrak{b} \int_{\Omega} \partial_{t}\left(1-\frac{z_{n, \sigma_{3}}}{\mathfrak{b}}\right) \mathcal{G}_{\sigma_{3}}^{\prime}\left(1-\frac{z_{n, \sigma_{3}}}{\mathfrak{b}}\right) \mathrm{d} x-\mathfrak{a} \int_{\Omega} \partial_{t} \mathbb{T}_{n, \sigma_{3}}: \mathcal{G}_{\sigma_{3}}^{\prime}\left(\mathbb{T}_{n, \sigma_{3}}\right) \mathrm{d} x \\
& =-\frac{\mathrm{d}}{\mathrm{d} t} \int_{\Omega}\left[\mathfrak{a} \operatorname{tr}\left(\mathcal{G}_{\sigma_{3}}\left(\mathbb{T}_{n, \sigma_{3}}\right)\right)+\mathfrak{b} \mathcal{G}_{\sigma_{3}}\left(1-\frac{z_{n, \sigma_{3}}}{\mathfrak{b}}\right)\right] \mathrm{d} x \\
& =-2 \frac{\mathrm{d}}{\mathrm{d} t} \int_{\Omega} \Pi_{\sigma_{3}}^{(2)}\left(\mathbb{T}_{n, \sigma_{3}}, z_{n, \sigma_{3}}\right) \mathrm{d} x \quad \text { a.e. on }\left(0, T_{n, \sigma_{3}}\right],
\end{aligned}
$$

where we have recalled (6.43). Similarly, we deduce from (6.30), (6.31) and Lemma 6.2 that

$$
\begin{gathered}
-\mathfrak{b} \int_{\Omega}\left(\mathbf{u}_{n, \sigma_{3}} \cdot \nabla_{x}\right) \chi_{\sigma_{3}}\left(1-\frac{z_{n, \sigma_{3}}}{\mathfrak{b}}\right) \mathcal{G}_{\sigma_{3}}^{\prime}\left(1-\frac{z_{n, \sigma_{3}}}{\mathfrak{b}}\right) \mathrm{d} x-\mathfrak{a} \int_{\Omega}\left(\mathbf{u}_{n, \sigma_{3}} \cdot \nabla_{x}\right) \chi_{\sigma_{3}}\left(\mathbb{T}_{n, \sigma_{3}}\right): \mathcal{G}_{\sigma_{3}}^{\prime}\left(\mathbb{T}_{n, \sigma_{3}}\right) \mathrm{d} x \\
=-\int_{\Omega} \mathbf{u}_{n, \sigma_{3}} \cdot \nabla_{x}\left[\mathfrak{a} \operatorname{tr}\left(\log \left(\chi_{\sigma_{3}}\left(\mathbb{T}_{n, \sigma_{3}}\right)\right)+\mathfrak{b} \log \chi_{\sigma_{3}}\left(1-\frac{z_{n, \sigma_{3}}}{\mathfrak{b}}\right)\right] \mathrm{d} x\right. \\
=\int_{\Omega}\left[\mathfrak{a} \operatorname{tr}\left(\log \left(\chi_{\sigma_{3}}\left(\mathbb{T}_{n, \sigma_{3}}\right)\right)+\mathfrak{b} \log \chi_{\sigma_{3}}\left(1-\frac{z_{n, \sigma_{3}}}{\mathfrak{b}}\right)\right] \mathbb{I}: \nabla_{x} \mathbf{u}_{n, \sigma_{3}} \mathrm{~d} x \quad \text { a.e. on }\left(0, T_{n, \sigma_{3}}\right] .\right.
\end{gathered}
$$

Next, by noting (6.32) and (1.9) for $\mathbf{u}_{n, \sigma_{3}}$, we have that

$$
\begin{aligned}
-2 \int_{\Omega} \operatorname{tr}\left(\nabla_{x} \mathbf{u}_{n, \sigma_{3}} \chi_{\sigma_{3}}\left(\mathbb{T}_{n, \sigma_{3}}\right)\right) \mathcal{G}_{\sigma_{3}}^{\prime}\left(1-\frac{z_{n, \sigma_{3}}}{\mathfrak{b}}\right) \mathrm{d} x & \\
& \quad+\mathfrak{a} \int_{\Omega}\left(\nabla_{x} \mathbf{u}_{n, \sigma_{3}} \chi_{\sigma_{3}}\left(\mathbb{T}_{n, \sigma_{3}}\right)+\chi_{\sigma_{3}}\left(\mathbb{T}_{n, \sigma_{3}}\right) \nabla_{x}^{\mathrm{T}} \mathbf{u}_{n, \sigma_{3}}\right): \mathcal{G}_{\sigma_{3}}^{\prime}\left(\mathbb{T}_{n, \sigma_{3}}\right) \mathrm{d} x \\
= & -2 \int_{\Omega}\left[\left[\chi_{\sigma_{3}}\left(1-\frac{z_{n, \sigma_{3}}}{\mathfrak{b}}\right)\right]^{-1} \operatorname{tr}\left(\chi_{\sigma_{3}}\left(\mathbb{T}_{n, \sigma_{3}}\right) \nabla_{x} \mathbf{u}_{n, \sigma_{3}}\right)-\mathfrak{a} \operatorname{div}_{x} \mathbf{u}_{n, \sigma_{3}}\right] \mathrm{d} x \\
= & -2 \int_{\Omega}\left[\chi_{\sigma_{3}}\left(1-\frac{z_{n, \sigma_{3}}}{\mathfrak{b}}\right)\right]^{-1} \chi_{\sigma_{3}}\left(\mathbb{T}_{n, \sigma_{3}}\right): \nabla_{x} \mathbf{u}_{n, \sigma_{3}} \mathrm{~d} x \quad \text { a.e. on }\left(0, T_{n, \sigma_{3}}\right] .
\end{aligned}
$$

We deduce from (6.32) and Lemma 7.1 that

$$
\begin{aligned}
-\varepsilon \int_{\Omega} \Delta_{x} z_{n, \sigma_{3}} \mathcal{G}_{\sigma_{3}}^{\prime}\left(1-\frac{z_{n, \sigma_{3}}}{\mathfrak{b}}\right) \mathrm{d} x+\varepsilon \mathfrak{a} \int_{\Omega} \Delta_{x} \mathbb{T}_{n, \sigma_{3}}: \mathcal{G}_{\sigma_{3}}^{\prime}\left(\mathbb{T}_{n, \sigma_{3}}\right) \mathrm{d} x \\
\quad=\varepsilon \mathfrak{b} \int_{\Omega} \Delta_{x}\left(1-\frac{z_{n, \sigma_{3}}}{\mathfrak{b}}\right)\left[\chi_{\sigma_{3}}\left(1-\frac{z_{n, \sigma_{3}}}{\mathfrak{b}}\right)\right]^{-1} \mathrm{~d} x+\varepsilon \mathfrak{a} \int_{\Omega} \Delta_{x} \mathbb{T}_{n, \sigma_{3}}:\left[\chi_{\sigma_{3}}\left(\mathbb{T}_{n, \sigma_{3}}\right)\right]^{-1} \mathrm{~d} x \\
\geq \varepsilon \int_{\Omega}\left[\mathfrak{b}\left|\nabla_{x} \log \left(\chi_{\sigma_{3}}\left(1-\frac{z_{n, \sigma_{3}}}{\mathfrak{b}}\right)\right)\right|^{2}+\frac{\mathfrak{a}}{d}\left|\nabla_{x} \operatorname{tr}\left(\log \chi_{\sigma_{3}}\left(\mathbb{T}_{n, \sigma_{3}}\right)\right)\right|^{2}\right] \mathrm{d} x \quad \text { a.e. on }\left(0, T_{n, \sigma_{3}}\right] .
\end{aligned}
$$


In addition, it follows from (6.32) and (6.39) that

$$
\begin{aligned}
-\frac{1}{2 \lambda} \int_{\Omega} \operatorname{tr}\left(\mathbb{A}_{n, \sigma_{3}}^{L}\right) \mathcal{G}_{\sigma_{3}}^{\prime}\left(1-\frac{z_{n, \sigma_{3}}}{\mathfrak{b}}\right) \mathrm{d} x+\frac{\mathfrak{a}}{2 \lambda} \int_{\Omega} \mathbb{A}_{n, \sigma_{3}}^{L}: \mathcal{G}_{\sigma_{3}}^{\prime}\left(\mathbb{T}_{n, \sigma_{3}}\right) \mathrm{d} x \\
=-\frac{1}{2 \lambda} \int_{\Omega} \mathbb{A}_{n, \sigma_{3}}^{L}:\left[\mathcal{G}_{\sigma_{3}}^{\prime}\left(1-\frac{z_{n, \sigma_{3}}}{\mathfrak{b}}\right) \mathbb{I}-\mathfrak{a} \mathcal{G}_{\sigma_{3}}^{\prime}\left(\mathbb{T}_{n, \sigma_{3}}\right)\right] \mathrm{d} x \\
=-\frac{1}{2 \lambda} \int_{\Omega} \operatorname{tr}\left(\left(\mathbb{A}_{n, \sigma_{3}}^{L}\right)^{2}\left[\chi_{\sigma_{3}}^{L}\left(\mathbb{T}_{n, \sigma_{3}}\right)\right]^{-1}\right) \mathrm{d} x \quad \text { a.e. on }\left(0, T_{n, \sigma_{3}}\right]
\end{aligned}
$$

Finally, we have from (6.32) and the boundary condition (1.9) for $\mathbf{u}_{n, \sigma_{3}}$ that

$$
\begin{aligned}
\mathfrak{b} \int_{\Omega} \operatorname{div}_{x} \mathbf{u}_{n, \sigma_{3}}\left[\chi_{\sigma_{3}}\left(1-\frac{z_{n, \sigma_{3}}}{\mathfrak{b}}\right)-\left(1-\frac{\operatorname{tr}\left(\chi_{\sigma_{3}}\left(\mathbb{T}_{n, \sigma_{3}}\right)\right)}{\mathfrak{b}}\right)\right] \mathcal{G}_{\sigma_{3}}^{\prime}\left(1-\frac{z_{n, \sigma_{3}}}{\mathfrak{b}}\right) \mathrm{d} x \\
=-\mathfrak{b} \int_{\Omega}\left[\chi_{\sigma_{3}}\left(1-\frac{z_{n, \sigma_{3}}}{\mathfrak{b}}\right)\right]^{-1}\left(1-\frac{\operatorname{tr}\left(\chi_{\sigma_{3}}\left(\mathbb{T}_{n, \sigma_{3}}\right)\right)}{\mathfrak{b}}\right) \mathbb{I}: \nabla_{x} \mathbf{u}_{n, \sigma_{3}} \mathrm{~d} x \quad \text { a.e. on }\left(0, T_{n, \sigma_{3}}\right]
\end{aligned}
$$

Combining (6.37), (6.38) and (7.7)-(7.12) yields, for a.e. on $\left(0, T_{n, \sigma_{3}}\right]$, that

$$
\begin{aligned}
& -2 \frac{\mathrm{d}}{\mathrm{d} t} \int_{\Omega} \Pi_{\sigma_{3}}^{(2)}\left(\mathbb{T}_{n, \sigma_{3}}, z_{n, \sigma_{3}}\right) \mathrm{d} x+\frac{1}{2 \lambda} \int_{\Omega} \operatorname{tr}\left(\left(\mathbb{A}_{n, \sigma_{3}}^{L}\right)^{2}\left[\chi_{\sigma_{3}}^{L}\left(\mathbb{T}_{n, \sigma_{3}}\right)\right]^{-1}\right) \mathrm{d} x \\
& \quad+\varepsilon \int_{\Omega}\left[\mathfrak{b}\left|\nabla_{x} \log \left(\chi_{\sigma_{3}}\left(1-\frac{z_{n, \sigma_{3}}}{\mathfrak{b}}\right)\right)\right|^{2}+\frac{\mathfrak{a}}{d}\left|\nabla_{x} \operatorname{tr}\left(\log \chi_{\sigma_{3}}\left(\mathbb{T}_{n, \sigma_{3}}\right)\right)\right|^{2}\right] \mathrm{d} x \\
& \leq 2 \int_{\Omega}\left[\left[\chi_{\sigma_{3}}\left(1-\frac{z_{n, \sigma_{3}}}{\mathfrak{b}}\right)\right]^{-1} \chi_{\sigma_{3}}\left(\mathbb{T}_{n, \sigma_{3}}\right)-\Pi_{\sigma_{3}}^{(1)}\left(\mathbb{T}_{n, \sigma_{3}}, z_{n, \sigma_{3}}\right) \mathbb{I}\right]: \nabla_{x} \mathbf{u}_{n, \sigma_{3}} \mathrm{~d} x .
\end{aligned}
$$

By adding $\frac{1}{2}(7.13)$ to $(7.6)$ we then obtain, for a.e. $t \in\left(0, T_{n, \sigma_{3}}\right]$, that

$$
\begin{aligned}
\frac{\mathrm{d}}{\mathrm{d} t} \int_{\Omega}\left[\frac{1}{2} \varrho_{n, \sigma_{3}}\left|\mathbf{u}_{n, \sigma_{3}}\right|^{2}+\frac{a}{\gamma-1} \varrho_{n, \sigma_{3}}^{\gamma}+\frac{\sigma_{1}}{\Gamma-1} \varrho_{n, \sigma_{3}}^{\Gamma}-\Pi_{\sigma_{3}}^{(2)}\left(\mathbb{T}_{n, \sigma_{3}}, z_{n, \sigma_{3}}\right)\right] \mathrm{d} x \\
\quad+4 \sigma_{2} \int_{\Omega}\left[\frac{a}{\gamma}\left|\nabla_{x} \varrho_{n, \sigma_{3}}^{\frac{\gamma}{2}}\right|^{2}+\frac{\sigma_{1}}{\Gamma}\left|\nabla_{x} \varrho_{n, \sigma_{3}}^{\frac{\Gamma}{2}}\right|^{2}\right] \mathrm{d} x+\frac{1}{4 \lambda} \int_{\Omega} \operatorname{tr}\left(\left(\mathbb{A}_{n, \sigma_{3}}^{L}\right)^{2}\left[\chi_{\sigma_{3}}^{L}\left(\mathbb{T}_{n, \sigma_{3}}\right)\right]^{-1}\right) \mathrm{d} x \\
\quad+\int_{\Omega} \mu^{S}\left[\left|\frac{\nabla_{x} \mathbf{u}_{n, \sigma_{3}}+\nabla_{x}^{\mathrm{T}} \mathbf{u}_{n, \sigma_{3}}}{2}-\frac{1}{d}\left(\operatorname{div}_{x} \mathbf{u}_{n, \sigma_{3}}\right) \mathbb{I}\right|^{2}+\mu^{B}\left|\operatorname{div}_{x} \mathbf{u}_{n, \sigma_{3}}\right|^{2}\right] \mathrm{d} x \\
\quad+\frac{\varepsilon}{2} \int_{\Omega}\left[\mathfrak{b}\left|\nabla_{x} \log \left(\chi_{\sigma_{3}}\left(1-\frac{z_{n, \sigma_{3}}}{\mathfrak{b}}\right)\right)\right|^{2}+\frac{\mathfrak{a}}{d}\left|\nabla_{x} \operatorname{tr}\left(\log \chi_{\sigma_{3}}\left(\mathbb{T}_{n, \sigma_{3}}\right)\right)\right|^{2}\right] \mathrm{d} x \\
\leq \int_{\Omega} \varrho_{n, \sigma_{3}} \mathbf{f} \cdot \mathbf{u}_{n, \sigma_{3}} \mathrm{~d} x
\end{aligned}
$$

As $\mathcal{G}_{\sigma_{3}}$ is concave and $\sigma_{3}<\theta<1$, recall (6.31), it follows from (6.43) and (6.42) that

$$
-\int_{\Omega} \Pi_{\sigma_{3}}^{(2)}\left(\mathbb{T}_{n, \sigma_{3}}, z_{n, \sigma_{3}}\right) \mathrm{d} x \geq \frac{1}{2} \int_{\Omega} \operatorname{tr}\left(\mathbb{T}_{n, \sigma_{3}}-\mathfrak{a} \mathcal{G}_{\sigma_{3}}\left(\mathbb{T}_{n, \sigma_{3}}\right)\right) \mathrm{d} x-\frac{\mathfrak{b}}{2}|\Omega|
$$

Given $\beta>0$, and since we can take $\sigma_{3}<\beta<\sigma_{3}^{-1}$, it is straightforward to check, by considering the four cases (i) $s \geq \sigma_{3}^{-1}$, (ii) $s \in\left[\sigma_{3}, \sigma_{3}^{-1}\right]$, (iii) $s \in\left[0, \sigma_{3}\right]$, and (iv) $s \leq 0$, that for any $s \in \mathbb{R}$, one has

$$
s-\beta \mathcal{G}_{\sigma_{3}}(s) \geq\left(1-\frac{\beta}{\sigma_{3}}\right)[s]_{-}+\beta-\beta \log \beta \geq \beta-\beta \log \beta,
$$

where $[s]_{-}:=\min \{s, 0\}$. Thus, we obtain by the diagonalisation process $(6.21)$ that

$$
\operatorname{tr}\left(\mathbb{P}-\beta \mathcal{G}_{\sigma_{3}}(\mathbb{P})\right)=\sum_{j=1}^{d}\left(\mathfrak{l}_{j}-\beta \mathcal{G}_{\sigma_{3}}\left(\mathfrak{l}_{j}\right)\right) \geq d(\beta-\beta \log \beta)
$$

Then, similarly as in (3.23), we define the following positive functional for $\sigma_{3}<\min \left\{\theta, \mathfrak{a}, \mathfrak{a}^{-1}\right\}$ :

$$
E_{n, \sigma_{3}}(t):=\int_{\Omega}\left[\frac{1}{2} \varrho_{n, \sigma_{3}}\left|\mathbf{u}_{n, \sigma_{3}}\right|^{2}+\frac{a}{\gamma-1} \varrho_{n, \sigma_{3}}^{\gamma}+\frac{\sigma_{1}}{\Gamma-1} \varrho_{n, \sigma_{3}}^{\Gamma}-\Pi_{\sigma_{3}}^{(2)}\left(\mathbb{T}_{n, \sigma_{3}}, z_{n, \sigma_{3}}\right)+\frac{1}{2}(d \mathfrak{a} \log \mathfrak{a}+\mathfrak{b})\right] \mathrm{d} x
$$


Similarly to (3.25), for $\sigma_{3}<\min \left\{\theta, \mathfrak{a}, \mathfrak{a}^{-1}\right\}$, integrating (7.14) over $[0, t]$, for any $t \in\left(0, T_{n, \sigma_{3}}\right]$, then gives

$$
\begin{aligned}
E_{n, \sigma_{3}}(t)+ & 4 \sigma_{2} \int_{0}^{t} \int_{\Omega}\left[\frac{a}{\gamma}\left|\nabla_{x} \varrho_{n, \sigma_{3}}^{\frac{\gamma}{2}}\right|^{2}+\frac{\sigma_{1}}{\Gamma}\left|\nabla_{x} \varrho_{n, \sigma_{3}}^{\frac{\Gamma}{2}}\right|^{2}\right] \mathrm{d} x \mathrm{~d} t^{\prime}+\frac{1}{4 \lambda} \int_{0}^{t} \int_{\Omega} \operatorname{tr}\left(\left(\mathbb{A}_{n, \sigma_{3}}^{L}\right)^{2}\left[\chi_{\sigma_{3}}^{L}\left(\mathbb{T}_{n, \sigma_{3}}\right)\right]^{-1}\right) \mathrm{d} x \mathrm{~d} t^{\prime} \\
& +\int_{0}^{t} \int_{\Omega}\left[\mu^{S}\left|\frac{\nabla_{x} \mathbf{u}_{n, \sigma_{3}}+\nabla_{x}^{\mathrm{T}} \mathbf{u}_{n, \sigma_{3}}}{2}-\frac{1}{d}\left(\operatorname{div}_{x} \mathbf{u}_{n, \sigma_{3}}\right) \mathbb{I}\right|^{2}+\mu^{B}\left|\operatorname{div}_{x} \mathbf{u}_{n, \sigma_{3}}\right|^{2}\right] \mathrm{d} x \mathrm{~d} t^{\prime} \\
& +\frac{\varepsilon}{2} \int_{0}^{t} \int_{\Omega}\left[\mathfrak{b}\left|\nabla_{x} \log \left(\chi_{\sigma_{3}}\left(1-\frac{z_{n, \sigma_{3}}}{\mathfrak{b}}\right)\right)\right|^{2}+\frac{\mathfrak{a}}{d}\left|\nabla_{x} \operatorname{tr}\left(\log \chi_{\sigma_{3}}\left(\mathbb{T}_{n, \sigma_{3}}\right)\right)\right|^{2}\right] \mathrm{d} x \mathrm{~d} t^{\prime} \\
\leq & E_{0, \sigma_{1}, \theta}+\int_{0}^{t} \int_{\Omega} \varrho_{n, \sigma_{3}} \mathbf{f} \cdot \mathbf{u}_{n, \sigma_{3}} \mathrm{~d} x \mathrm{~d} t^{\prime} \leq E_{0, \sigma_{1}, \theta}+C \int_{0}^{t} E_{n, \sigma_{3}}\left(t^{\prime}\right) \mathrm{d} t^{\prime},
\end{aligned}
$$

where the initial positive energy $E_{0, \sigma_{1}, \theta}$ is defined as

$$
E_{0, \sigma_{1}, \theta}:=\int_{\Omega}\left[\frac{1}{2} \varrho_{0, \theta}\left|\mathbf{u}_{0, \theta}\right|^{2}+\frac{a}{\gamma-1} \varrho_{0, \theta}^{\gamma}+\frac{\sigma_{1}}{\Gamma-1} \varrho_{0, \theta}^{\Gamma}-\Pi^{(2)}\left(\mathbb{T}_{0, \theta}\right)+\frac{1}{2}(d \mathfrak{a} \log \mathfrak{a}+\mathfrak{b})\right] \mathrm{d} x .
$$

Since we can choose $\sigma_{3}^{-1}>\mathfrak{b}^{\star}>\tau_{\min }>\sigma_{3}$, by recalling (3.1) $)_{3}$, and as $\theta>\sigma_{3}$, by recalling (6.30), we have for any $\theta \in\left(0, \frac{\mathfrak{b}-\mathfrak{b}^{\star}}{\mathfrak{b}}\right)$, on noting $(6.6)$, that

$$
\begin{aligned}
& \sigma_{3} \mathbb{I}<\tau_{\min } \mathbb{I} \leq \mathbb{T}_{0, \theta} \leq \mathfrak{b}^{\star} \mathbb{I}<\sigma_{3}^{-1} \mathbb{I} \quad \Rightarrow \quad \operatorname{tr}\left(\mathcal{G}_{\sigma_{3}}\left(\mathbb{T}_{0, \theta}\right)\right) \equiv \operatorname{tr}\left(\log \mathbb{T}_{0, \theta}\right) \geq d \log \tau_{\min } \\
& \text { and } \quad 0 \leq \operatorname{tr}\left(\mathbb{T}_{0, \theta}\right) \leq \mathfrak{b}^{\star}<\mathfrak{b}(1-\theta)<\mathfrak{b}\left(1-\sigma_{3}\right) \Rightarrow\left\{\begin{aligned}
\mathcal{G}_{\sigma_{3}}\left(1-\frac{\operatorname{tr}\left(\mathbb{T}_{0, \theta}\right)}{\mathfrak{b}}\right) & \equiv \log \left(1-\frac{\operatorname{tr}\left(\mathbb{T}_{0, \theta}\right)}{\mathfrak{b}}\right) \\
& \geq \log \left(1-\frac{\mathfrak{b}^{\star}}{\mathfrak{b}}\right) .
\end{aligned}\right.
\end{aligned}
$$

Then, Gronwall's inequality applied to (7.19), for $\sigma_{3}<\min \left\{\theta, \mathfrak{a}, \mathfrak{a}^{-1}, \tau_{\min },\left(\mathfrak{b}^{\star}\right)^{-1}\right\}$, implies, for any $t \in\left(0, T_{n, \sigma_{3}}\right]$, that

$$
\begin{aligned}
E_{n, \sigma_{3}}(t)+ & 4 \sigma_{2} \int_{0}^{t} \int_{\Omega}\left(\frac{a}{\gamma}\left|\nabla_{x} \varrho_{n, \sigma_{3}}^{\frac{\gamma}{2}}\right|^{2}+\frac{\sigma_{1}}{\Gamma}\left|\nabla_{x} \varrho_{n, \sigma_{3}}^{\frac{\Gamma}{2}}\right|^{2}\right) \mathrm{d} x \mathrm{~d} t^{\prime}+\frac{1}{4 \lambda} \int_{0}^{t} \int_{\Omega} \operatorname{tr}\left(\left(\mathbb{A}_{n, \sigma_{3}}^{L}\right)^{2}\left[\chi_{\sigma_{3}}^{L}\left(\mathbb{T}_{n, \sigma_{3}}\right)\right]^{-1}\right) \mathrm{d} x \mathrm{~d} t^{\prime} \\
& +\int_{0}^{t} \int_{\Omega}\left[\mu\left|\frac{\nabla_{x} \mathbf{u}_{n, \sigma_{3}}+\nabla_{x}^{\mathrm{T}} \mathbf{u}_{n, \sigma_{3}}}{2}-\frac{1}{d}\left(\operatorname{div}_{x} \mathbf{u}_{n, \sigma_{3}}\right) \mathbb{I}\right|^{2}+\mu^{B}\left|\operatorname{div}_{x} \mathbf{u}_{n, \sigma_{3}}\right|^{2}\right] \mathrm{d} x \mathrm{~d} t^{\prime} \\
& +\frac{\varepsilon}{2} \int_{0}^{t} \int_{\Omega}\left[\mathfrak{b}\left|\nabla_{x} \log \left(\chi_{\sigma_{3}}\left(1-\frac{z_{n, \sigma_{3}}}{\mathfrak{b}}\right)\right)\right|^{2}+\frac{\mathfrak{a}}{d}\left|\nabla_{x} \operatorname{tr}\left(\log \chi_{\sigma_{3}}\left(\mathbb{T}_{n, \sigma_{3}}\right)\right)\right|^{2}\right] \mathrm{d} x \mathrm{~d} t^{\prime} \\
\leq & E_{0, \sigma_{1}, \theta}+\int_{0}^{t} \int_{\Omega} \varrho_{n, \sigma_{3}} \mathbf{f} \cdot \mathbf{u}_{n, \sigma_{3}} \mathrm{~d} x \mathrm{~d} t^{\prime} \leq E_{0, \sigma_{1}, \theta}+C \int_{0}^{t} E_{n, \sigma_{3}}\left(t^{\prime}\right) \mathrm{d} t^{\prime} \\
\leq & \mathrm{e}^{C t} E_{0, \sigma_{1}, \theta}
\end{aligned}
$$

where $C$ is a positive constant, independent of $t$ and of the approximation parameters $\left(\Gamma, \sigma_{1}, \sigma_{2}, \sigma_{3}, \theta, L, n\right)$ and, for the above constraint on $\sigma_{3}$, it follows from (7.20), (6.43), (1.8) and (7.21) that

$$
\begin{aligned}
E_{0, \sigma_{1}, \theta} & =\int_{\Omega}\left[\frac{1}{2} \varrho_{0, \theta}\left|\mathbf{u}_{0, \theta}\right|^{2}+\frac{a}{\gamma-1} \varrho_{0, \theta}^{\gamma}+\frac{\sigma_{1}}{\Gamma-1} \varrho_{0, \theta}^{\Gamma}-\Pi\left(\mathbb{T}_{0, \theta}\right)+\frac{1}{2}(d \mathfrak{a} \log \mathfrak{a}+\mathfrak{b})\right] \mathrm{d} x \\
& \leq \int_{\Omega}\left[\frac{1}{2} \varrho_{0, \theta}\left|\mathbf{u}_{0, \theta}\right|^{2}+\frac{a}{\gamma-1} \varrho_{0, \theta}^{\gamma}+\frac{\sigma_{1}}{\Gamma-1} \varrho_{0, \theta}^{\Gamma}+\frac{d \mathfrak{a}}{2} \log \left(\frac{\mathfrak{a}}{\tau_{\min }}\right)-\frac{\mathfrak{b}}{2} \log \left(1-\frac{\mathfrak{b}^{\star}}{\mathfrak{b}}\right)\right] \mathrm{d} x .
\end{aligned}
$$

Hence, $E_{0, \sigma_{1}, \theta}$ is finite for any $\theta \in\left(0, \frac{\mathfrak{b}-\mathfrak{b}^{\star}}{\mathfrak{b}}\right)$ by noting $(6.1)_{1,2}$ and (6.2).

Remark 7.2. Instead of (6.37) and (6.38), one could adopt the more straightforward approximation based on just

$$
\begin{aligned}
\partial_{t} \mathbb{T}_{n, \sigma_{3}}+\left(\mathbf{u}_{n, \sigma_{3}} \cdot \nabla_{x}\right) \mathbb{T}_{n, \sigma_{3}}-\left(\nabla_{x} \mathbf{u}_{n, \sigma_{3}} \chi_{\sigma_{3}}\left(\mathbb{T}_{n, \sigma_{3}}^{S}\right)+\chi_{\sigma_{3}}\left(\mathbb{T}_{n, \sigma_{3}}^{S}\right) \nabla_{x}^{\mathrm{T}} \mathbf{u}_{n, \sigma_{3}}\right) \\
=\varepsilon \Delta_{x} \mathbb{T}_{n, \sigma_{3}}-\frac{1}{2 \lambda}\left(\left[\chi_{\sigma_{3}}\left(1-\frac{\operatorname{tr}\left(\mathbb{T}_{n, \sigma_{3}}^{S}\right)}{\mathfrak{b}}\right)\right]^{-1} \mathbb{I}-\mathfrak{a}\left[\chi_{\sigma_{3}}\left(\mathbb{T}_{n, \sigma_{3}}^{S}\right)\right]^{-1}\right) \chi_{\sigma_{3}}^{L}\left(\mathbb{T}_{n, \sigma_{3}}^{S}\right) .
\end{aligned}
$$

Once again it follows that $\mathbb{T}_{n, \sigma_{3}}=\mathbb{T}_{n, \sigma_{3}}^{S}$. To mimic the testing procedure of (1.3) with (3.8), by recalling (6.32) and (6.30), one would test $(7.24)$ with $\mathcal{G}_{\sigma_{3}}^{\prime}\left(1-\frac{\operatorname{tr}\left(\mathbb{T}_{n, \sigma_{3}}\right)}{\mathfrak{b}}\right)-\mathfrak{a} \mathcal{G}_{\sigma_{3}}^{\prime}\left(\mathbb{T}_{n, \sigma_{3}}\right)$. In order to achieve the analogue of the (7.14), we 
would have to replace $\Pi_{\sigma_{3}}^{(2)}\left(\mathbb{T}_{n, \sigma_{3}}, z_{n, \sigma_{3}}\right)$ in (7.14) and $\Pi_{\sigma_{3}}^{(1)}\left(\mathbb{T}_{n, \sigma_{3}}, z_{n, \sigma_{3}}\right)$ in (6.33) by

$$
\Pi_{\sigma_{3}}\left(\mathbb{T}_{n, \sigma_{3}}\right)=\frac{1}{2}\left[\mathfrak{a} \operatorname{tr}\left(\mathcal{G}_{\sigma_{3}}\left(\mathbb{T}_{n, \sigma_{3}}\right)\right)+\mathfrak{b} \mathcal{G}_{\sigma_{3}}\left(1-\frac{\operatorname{tr}\left(\mathbb{T}_{n, \sigma_{3}}\right)}{\mathfrak{b}}\right)+\mathfrak{b}\right] .
$$

However, we are not able to prove the analogue of (8.38) $)_{3}$ below for the polymeric pressure approximation (7.25). Although, one can prove convergence almost everywhere, using the analogues of $(8.2)_{2}$ and (8.18) below, we are still unable to prove the analogue of $(8.38)_{3}$ below even if "strongly" were replaced by "weakly" and $r=1$.

7.2. Maximal existence time. In this section, we shall use the uniform bound (7.22) to show that $T_{n, \sigma_{3}}$, the maximal time of existence for solutions to the fourth level of approximation, is in fact equal to the final time $T$.

By Friedrichs' inequality and Korn's inequality (3.28), a partial result from the bound (7.22) is that

$$
\int_{0}^{T_{n, \sigma_{3}}}\left\|\mathbf{u}_{n, \sigma_{3}}(t)\right\|_{W^{1,2}\left(\Omega ; \mathbb{R}^{d}\right)}^{2} \mathrm{~d} t \leq C \int_{0}^{T_{n, \sigma_{3}}}\left\|\nabla_{x} \mathbf{u}_{n, \sigma_{3}}(t)\right\|_{L^{2}\left(\Omega ; \mathbb{R}^{d \times d}\right)}^{2} \mathrm{~d} t \leq \mathrm{e}^{C T_{n, \sigma_{3}}} E_{0, \sigma_{1}, \theta} \leq C\left(E_{0, \sigma_{1}, \theta}, T\right) .
$$

By the equivalence of the $W^{1,2}(\Omega)$ and $W^{1, \infty}(\Omega)$ norms in the finite-dimensional linear space $X_{n}$ (see $(6.17)$ ), and the Cauchy-Schwarz inequality over $\left(0, T_{n, \sigma_{3}}\right]$, we then have from (7.26) that

$$
\int_{0}^{T_{n, \sigma_{3}}}\left\|\nabla_{x} \mathbf{u}_{n, \sigma_{3}}(t)\right\|_{L^{\infty}\left(\Omega ; \mathbb{R}^{d \times d}\right)} \mathrm{d} t \leq C\left(n, E_{0, \sigma_{1}, \theta}, T\right) .
$$

Using (7.27) it follows from $(7.2)_{3}$ that we have the following lower and upper bounds on $\varrho_{n, \sigma_{3}}$ in terms of positive constants:

$$
C\left(\theta, n, E_{0, \sigma_{1}, \theta}, T\right)^{-1} \leq \varrho_{n, \sigma_{3}}(t, x) \leq C\left(\theta, n, E_{0, \sigma_{1}, \theta}, T\right), \quad \text { for all }(t, x) \in\left(0, T_{n, \sigma_{3}}\right] \times \Omega .
$$

Together with the following partial result from (7.22) and (7.18):

$$
\sup _{t \in\left(0, T_{n, \sigma_{3}}\right]}\left\|\varrho_{n, \sigma_{3}}\left|\mathbf{u}_{n, \sigma_{3}}\right|^{2}(t)\right\|_{L^{1}(\Omega)} \leq C\left(E_{0, \sigma_{1}, \theta}, T\right),
$$

we obtain

$$
\sup _{t \in\left(0, T_{n, \sigma_{3}}\right]}\left\|\mathbf{u}_{n, \sigma_{3}}(t)\right\|_{L^{2}\left(\Omega ; \mathbb{R}^{d}\right)} \leq C\left(\theta, n, E_{0, \sigma_{1}, \theta}, T\right) .
$$

Again by the properties in (6.17) of functions in $X_{n}$, we have

$$
\sup _{t \in\left(0, T_{n, \sigma_{3}}\right]}\left\|\mathbf{u}_{n, \sigma_{3}}(t)\right\|_{C^{2, \beta}\left(\bar{\Omega} ; \mathbb{R}^{d}\right)} \leq C\left(\theta, n, E_{0, \sigma_{1}, \theta}, T\right) .
$$

Hence, by a continuity argument, the existence time can exceed $T_{n, \sigma_{3}}$. Since the bound in (7.22) is independent of $n, \sigma_{3}$, this process can be repeated a finite number of times, as long as the existence time $T_{n, \sigma_{3}}<T$, until the final time $T$ is reached, and therefore the maximal existence time $T_{n, \sigma_{3}}=T$. Moreover, by (7.2) and (7.28), (see also the paragraph after (7.29)), we have the following bounds:

(7.29)

$$
\begin{aligned}
& \sup _{t \in(0, T]}\left\|\mathbf{u}_{n, \sigma_{3}}(t)\right\|_{C^{2, \beta}\left(\bar{\Omega} ; \mathbb{R}^{d}\right)} \leq C\left(\theta, n, E_{0, \sigma_{1}, \theta}, T\right), \\
& C\left(\theta, n, E_{0, \sigma_{1}, \theta}, T\right)^{-1} \leq \varrho_{n, \sigma_{3}}(t, x) \leq C\left(\theta, n, E_{0, \sigma_{1}, \theta}, T\right), \quad \text { for all }(t, x) \in(0, T] \times \Omega, \\
& \sup _{t \in(0, T]}\left\|\varrho_{n, \sigma_{3}}(t)\right\|_{W^{1,2}(\Omega)}+\left\|\varrho_{n, \sigma_{3}}\right\|_{L^{2}\left(0, T ; W^{2,2}(\Omega)\right)}+\left\|\partial_{t} \varrho_{n, \sigma_{3}}\right\|_{L^{2}\left(0, T ; L^{2}(\Omega)\right)} \leq C\left(\sigma_{2}, \theta, n, E_{0, \sigma_{1}, \theta}, T\right), \\
& \sup _{t \in(0, T]}\left\|\mathbb{T}_{n, \sigma_{3}}(t)\right\|_{W^{1,2}\left(\Omega ; \mathbb{R}^{d \times d}\right)}+\left\|\mathbb{T}_{n, \sigma_{3}}\right\|_{L^{2}\left(0, T ; W^{2,2}\left(\Omega ; \mathbb{R}^{d \times d}\right)\right)}+\left\|\partial_{t} \mathbb{T}_{n, \sigma_{3}}\right\|_{L^{2}\left(0, T ; L^{2}\left(\Omega ; \mathbb{R}^{d \times d}\right)\right)} \leq C\left(\sigma_{3}, L, \theta, n, E_{0, \sigma_{1}, \theta}, T\right), \\
& \sup _{t \in(0, T]}\left\|z_{n, \sigma_{3}}(t)\right\|_{W^{1,2}(\Omega)}+\left\|z_{n, \sigma_{3}}\right\|_{L^{2}\left(0, T ; W^{2,2}(\Omega)\right)}+\left\|\partial_{t} z_{n, \sigma_{3}}\right\|_{L^{2}\left(0, T ; L^{2}(\Omega)\right)} \leq C\left(\sigma_{3}, L, \theta, n, E_{0, \sigma_{1}, \theta}, T\right) .
\end{aligned}
$$

Here the bound on $\partial_{t} \varrho_{n, \sigma_{3}}$ follows from (6.36), $(7.29)_{1}$ and the second bound in $(7.29)_{3}$. Similarly, the bounds on $\partial_{t} \mathbb{T}_{n, \sigma_{3}}$ and $\partial_{t} z_{n, \sigma_{3}}$ follow from (6.37), (6.38), (7.29) $)_{1}$, the second bounds in (7.29) $)_{4,5}$ and (7.3). Whereas the bounds on $\mathbf{u}_{n, \sigma_{3}}$ and $\varrho_{n, \sigma_{3}}$ in (7.29) are independent of $\sigma_{3}$ and $L$, the bounds on $\mathbb{T}_{n, \sigma_{3}}$ and $z_{n, \sigma_{3}}$ are not, as they depend on (7.3). In the next section we establish analogous bounds on $\mathbb{T}_{n, \sigma_{3}}$ and $z_{n, \sigma_{3}}$, that are independent of $\sigma_{3}$.

In order to achieve this, we first note from (7.18) and (7.22), for time $T$ as opposed to $T_{n, \sigma_{3}}$, that the following bounds are also uniform with respect to $\sigma_{3}$ and $L$ :

$$
\begin{aligned}
\sup _{t \in(0, T]} \int_{\Omega} & {\left[-\Pi_{\sigma_{3}}^{(2)}\left(\mathbb{T}_{n, \sigma_{3}}, z_{n, \sigma_{3}}\right)+\frac{1}{2}(d \mathfrak{a} \log \mathfrak{a}+\mathfrak{b})\right] \mathrm{d} x \leq C\left(E_{0, \sigma_{1}, \theta}, T\right), } \\
& \int_{0}^{T} \int_{\Omega} \operatorname{tr}\left(\left(\mathbb{A}_{n, \sigma_{3}}^{L}\right)^{2}\left[\chi_{\sigma_{3}}^{L}\left(\mathbb{T}_{n, \sigma_{3}}\right)\right]^{-1}\right) \mathrm{d} x \mathrm{~d} t \leq C\left(E_{0, \sigma_{1}, \theta}, T\right) .
\end{aligned}
$$


Similarly to (3.31), as $\chi_{\sigma_{3}}^{L}\left(\mathbb{T}_{n, \sigma_{3}}\right)$ is symmetric positive definite, it follows from (6.30) and $(7.30)_{2}$ that

$$
\begin{aligned}
\left\|\mathbb{A}_{n, \sigma_{3}}^{L}\right\|_{L^{2}\left(0, T ; L^{2}\left(\Omega, \mathbb{R}^{d \times d}\right)\right)}^{2} & =\int_{0}^{T} \int_{\Omega}\left|\mathbb{A}_{n, \sigma_{3}}^{L}\left[\chi_{\sigma_{3}}^{L}\left(\mathbb{T}_{n, \sigma_{3}}\right)\right]^{-\frac{1}{2}}\left[\chi_{\sigma_{3}}^{L}\left(\mathbb{T}_{n, \sigma_{3}}\right)\right]^{\frac{1}{2}}\right|^{2} \mathrm{~d} x \mathrm{~d} t \\
& \leq d L \int_{0}^{T} \int_{\Omega} \operatorname{tr}\left(\left(\mathbb{A}_{n, \sigma_{3}}^{L}\right)^{2}\left[\chi_{\sigma_{3}}^{L}\left(\mathbb{T}_{n, \sigma_{3}}\right)\right]^{-1}\right) \leq C\left(L, E_{0, \sigma_{1}, \theta}, T\right) .
\end{aligned}
$$

7.3. Bounds on $\mathbb{T}_{n, \sigma_{3}}$ and $z_{n, \sigma_{3}}$ independent of $\sigma_{3}$. Similarly to (3.32), taking the inner product of (6.37) with $\mathbb{T}_{n, \sigma_{3}}$, integrating over $\Omega$, performing partial integration and noting the symmetry of $\mathbb{T}_{n, \sigma_{3}}$, the boundary conditions (1.9), (1.10) for $\mathbf{u}_{n, \sigma_{3}}$ and $\mathbb{T}_{n, \sigma_{3}},(7.29)_{1},(6.30)$ and (7.31) yield

$$
\begin{aligned}
\frac{1}{2} \frac{\mathrm{d}}{\mathrm{d} t} & \int_{\Omega}\left|\mathbb{T}_{n, \sigma_{3}}\right|^{2} \mathrm{~d} x+\varepsilon \int_{\Omega}\left|\nabla_{x} \mathbb{T}_{n, \sigma_{3}}\right|^{2} \mathrm{~d} x \\
= & \int_{\Omega} \mathbf{F}_{n, \sigma_{3}}: \mathbb{T}_{n, \sigma_{3}} \mathrm{~d} x \\
= & \int_{\Omega}\left[\left(\operatorname{div}_{x} \mathbf{u}_{n, \sigma_{3}}\right) \chi_{\sigma_{3}}\left(\mathbb{T}_{n, \sigma_{3}}\right): \mathbb{T}_{n, \sigma_{3}}+\chi_{\sigma_{3}}\left(\mathbb{T}_{n, \sigma_{3}}\right):\left(\mathbf{u}_{n, \sigma_{3}} \cdot \nabla_{x}\right) \mathbb{T}_{n, \sigma_{3}}\right] \mathrm{d} x \\
& \quad+\int_{\Omega}\left(\nabla_{x} \mathbf{u}_{n, \sigma_{3}} \chi_{\sigma_{3}}\left(\mathbb{T}_{n, \sigma_{3}}\right)+\chi_{\sigma_{3}}\left(\mathbb{T}_{n, \sigma_{3}}\right) \nabla_{x}^{\mathrm{T}} \mathbf{u}_{n, \sigma_{3}}\right): \mathbb{T}_{n, \sigma_{3}} \mathrm{~d} x-\frac{1}{2 \lambda} \int_{\Omega} \mathbb{A}_{n, \sigma_{3}}^{L}: \mathbb{T}_{n, \sigma_{3}} \mathrm{~d} x \\
\leq & \frac{\varepsilon}{2} \int_{\Omega}\left|\nabla_{x} \mathbb{T}_{n, \sigma_{3}}\right|^{2} \mathrm{~d} x+C\left(L, E_{0, \sigma_{1}, \theta}, T\right)\left(1+\left\|\mathbf{u}_{n, \sigma_{3}}\right\|_{W^{1, \infty}\left(\Omega ; \mathbb{R}^{d}\right)}^{2}\right)\left(1+\int_{\Omega}\left|\mathbb{T}_{n, \sigma_{3}}\right|^{2} \mathrm{~d} x\right) \\
\leq & \frac{\varepsilon}{2} \int_{\Omega}\left|\nabla_{x} \mathbb{T}_{n, \sigma_{3}}\right|^{2} \mathrm{~d} x+C\left(L, \theta, n, E_{0, \sigma_{1}, \theta}, T\right)\left(1+\int_{\Omega}\left|\mathbb{T}_{n, \sigma_{3}}\right|^{2} \mathrm{~d} x\right), \quad \text { for a.e. } t \in(0, T] .
\end{aligned}
$$

Rearranging and applying a Gronwall inequality to (7.32) and noting the initial condition (6.3) satisfies (6.6), we obtain the following bound on $\mathbb{T}_{n, \sigma_{3}}$, which is uniform in $\sigma_{3}$ :

$$
\sup _{t \in(0, T]}\left\|\mathbb{T}_{n, \sigma_{3}}(t)\right\|_{L^{2}\left(\Omega ; \mathbb{R}^{d \times d}\right)}+\left\|\mathbb{T}_{n, \sigma_{3}}\right\|_{L^{2}\left(0, T ; W^{1,2}\left(\Omega ; \mathbb{R}^{d \times d}\right)\right)} \leq C\left(L, \theta, n, E_{0, \sigma_{1}, \theta}, T\right) .
$$

Obviously, on recalling (6.30), similar bounds hold for $\chi_{\sigma_{3}}\left(\mathbb{T}_{n, \sigma_{3}}\right)$.

Similarly to (7.32), multiplying (6.38) with $z_{n, \sigma_{3}}$, integrating over $\Omega$, performing partial integration and noting the symmetry of $\mathbb{T}_{n, \sigma_{3}}$, the boundary conditions (1.9) for $\mathbf{u}_{n, \sigma_{3}},(6.40),(7.29)_{1},(6.30),(7.31)$ and (7.33) yield

$$
\begin{aligned}
\frac{1}{2} \frac{\mathrm{d}}{\mathrm{d} t} & \int_{\Omega}\left|z_{n, \sigma_{3}}\right|^{2} \mathrm{~d} x+\varepsilon \int_{\Omega}\left|\nabla_{x} z_{n, \sigma_{3}}\right|^{2} \mathrm{~d} x \\
= & \int_{\Omega} F_{z, n, \sigma_{3}} z_{n, \sigma_{3}} \mathrm{~d} x \\
= & -\mathfrak{b} \int_{\Omega} \chi_{\sigma_{3}}\left(1-\frac{z_{n, \sigma_{3}}}{\mathfrak{b}}\right)\left(\mathbf{u}_{n, \sigma_{3}} \cdot \nabla_{x} z_{n, \sigma_{3}}\right) \mathrm{d} x-\mathfrak{b} \int_{\Omega}\left(\operatorname{div}_{x} \mathbf{u}_{n, \sigma_{3}}\right)\left(1-\frac{\operatorname{tr}\left(\chi_{\sigma_{3}}\left(\mathbb{T}_{n, \sigma_{3}}\right)\right)}{\mathfrak{b}}\right) z_{n, \sigma_{3}} \mathrm{~d} x \\
& \quad+2 \int_{\Omega} \operatorname{tr}\left(\nabla_{x} \mathbf{u}_{n, \sigma_{3}} \chi_{\sigma_{3}}\left(\mathbb{T}_{n, \sigma_{3}}\right)\right) z_{n, \sigma_{3}} \mathrm{~d} x-\frac{1}{2 \lambda} \int_{\Omega} \operatorname{tr}\left(\mathbb{A}_{n, \sigma_{3}}^{L}\right) z_{n, \sigma_{3}} \mathrm{~d} x \\
\leq & \frac{\varepsilon}{2} \int_{\Omega}\left|\nabla_{x} z_{n, \sigma_{3}}\right|^{2} \mathrm{~d} x+C\left(L, \theta, n, E_{0, \sigma_{1}, \theta}, T\right)\left(1+\left\|\mathbf{u}_{n, \sigma_{3}}\right\|_{W^{1, \infty}\left(\Omega ; \mathbb{R}^{d}\right)}^{2}+\left\|\mathbb{T}_{n, \sigma_{3}}\right\|_{L^{2}\left(\Omega ; \mathbb{R}^{d \times d}\right)}^{2}\right)\left(1+\int_{\Omega}\left|z_{n, \sigma_{3}}\right|^{2} \mathrm{~d} x\right) \\
\leq & \frac{\varepsilon}{2} \int_{\Omega}\left|\nabla_{x} z_{n, \sigma_{3}}\right|^{2} \mathrm{~d} x+C\left(L, \theta, n, E_{0, \sigma_{1}, \theta}, T\right)\left(1+\int_{\Omega}\left|z_{n, \sigma_{3}}\right|^{2} \mathrm{~d} x\right) .
\end{aligned}
$$

Rearranging and applying a Gronwall inequality to (7.34) and noting (6.40) and (6.6), we obtain the following bound on $z_{n, \sigma_{3}}$, which is uniform in $\sigma_{3}$ :

$$
\sup _{t \in(0, T]}\left\|z_{n, \sigma_{3}}(t)\right\|_{L^{2}(\Omega)}+\left\|z_{n, \sigma_{3}}\right\|_{L^{2}\left(0, T ; W^{1,2}(\Omega)\right)} \leq C\left(L, \theta, n, E_{0, \sigma_{1}, \theta}, T\right) .
$$

Obviously, on recalling (6.30), similar bounds hold for $\chi_{\sigma_{3}}\left(1-\frac{z_{n, \sigma_{3}}}{\mathfrak{b}}\right)$.

It follows from $(6.37),(6.38),(7.29)_{1},(7.31),(6.30),(6.34),(7.33)$ and $(7.35)$ that

$$
\begin{aligned}
& \left\|\mathbf{F}_{n, \sigma_{3}}\right\|_{L^{2}\left(0, T ; L^{2}\left(\Omega ; \mathbb{R}^{d \times d}\right)\right)}+\left\|F_{z, n, \sigma_{3}}\right\|_{L^{2}\left(0, T ; L^{2}(\Omega)\right)} \\
& \quad \leq C\left(\theta, n, E_{0, \sigma_{1}, \theta}, T\right)\left(1+\left\|\mathbb{A}_{n, \sigma_{3}}^{L}\right\|_{L^{2}\left(0, T ; L^{2}\left(\Omega ; \mathbb{R}^{d \times d}\right)\right)}+\left\|\mathbb{T}_{n, \sigma_{3}}\right\|_{L^{2}\left(0, T ; W^{1,2}\left(\Omega ; \mathbb{R}^{d \times d}\right)\right)}+\left\|z_{n, \sigma_{3}}\right\|_{L^{2}\left(0, T ; W^{1,2}(\Omega)\right)}\right) \\
& \quad \leq C\left(L, \theta, n, E_{0, \sigma_{1}, \theta}, T\right) .
\end{aligned}
$$


By noting (7.36), the boundary and initial conditions (1.10), (6.3) for $\mathbb{T}_{n, \sigma_{3}},(6.40)$ and (6.6), we now apply Lemma 5.6 to both (6.37) and (6.38) to obtain the following bounds, which are uniform in $\sigma_{3}$ :

$$
\begin{aligned}
& \left\|\mathbb{T}_{n, \sigma_{3}}\right\|_{L^{\infty}\left(0, T ; W^{1,2}\left(\Omega ; \mathbb{R}^{d \times d}\right)\right)}+\left\|\mathbb{T}_{n, \sigma_{3}}\right\|_{L^{2}\left(0, T ; W^{2,2}\left(\Omega ; \mathbb{R}^{d \times d}\right)\right)}+\left\|\partial_{t} \mathbb{T}_{n, \sigma_{3}}\right\|_{L^{2}\left(0, T ; L^{2}\left(\Omega ; \mathbb{R}^{d \times d}\right)\right)} \\
& \quad+\left\|z_{n, \sigma_{3}}\right\|_{L^{\infty}\left(0, T ; W^{1,2}(\Omega)\right)}+\left\|z_{n, \sigma_{3}}\right\|_{L^{2}\left(0, T ; W^{2,2}(\Omega)\right)}+\left\|\partial_{t} z_{n, \sigma_{3}}\right\|_{L^{2}\left(0, T ; L^{2}(\Omega)\right)} \leq C\left(L, \theta, n, E_{0, \sigma_{1}, \theta}, T\right) .
\end{aligned}
$$

We deduce from (7.37), by applying Sobolev embedding for $d \leq 3$, the following bounds, which are uniform in $\sigma_{3}$ :

$$
\begin{aligned}
& \left\|\mathbb{T}_{n, \sigma_{3}}\right\|_{L^{\infty}\left(0, T ; L^{6}\left(\Omega ; \mathbb{R}^{d \times d}\right)\right)}+\left\|\mathbb{T}_{n, \sigma_{3}}\right\|_{L^{2}\left(0, T ; W^{1,6}\left(\Omega ; \mathbb{R}^{d \times d}\right)\right)} \\
& \quad+\left\|z_{n, \sigma_{3}}\right\|_{L^{\infty}\left(0, T ; L^{6}(\Omega)\right)}+\left\|z_{n, \sigma_{3}}\right\|_{L^{2}\left(0, T ; W^{1,6}(\Omega)\right)} \leq C\left(L, \theta, n, E_{0, \sigma_{1}, \theta}, T\right) .
\end{aligned}
$$

It follows from $(6.41),(7.29)_{1},(6.30),(6.34)$ and $(7.38)$ that

$$
\begin{aligned}
\left\|F_{z, n, \sigma_{3}}-\operatorname{tr}\left(\mathbf{F}_{n, \sigma_{3}}\right)\right\|_{L^{2}\left(0, T ; L^{6}(\Omega)\right)} & \leq C\left(\theta, n, E_{0, \sigma_{1}, \theta}, T\right)\left(1+\left\|\mathbb{T}_{n, \sigma_{3}}\right\|_{L^{2}\left(0, T ; W^{1,6}\left(\Omega ; \mathbb{R}^{d \times d}\right)\right)}+\left\|z_{n, \sigma_{3}}\right\|_{L^{2}\left(0, T ; W^{1,6}(\Omega)\right)}\right) \\
& \leq C\left(L, \theta, n, E_{0, \sigma_{1}, \theta}, T\right) .
\end{aligned}
$$

By noting (7.39), the boundary conditions (1.10) for $\mathbb{T}_{n, \sigma_{3}}$ and (6.40), we now apply Lemma 5.6 to (6.41) to obtain that

$$
\left\|z_{n, \sigma_{3}}-\operatorname{tr}\left(\mathbb{T}_{n, \sigma_{3}}\right)\right\|_{L^{\infty}\left(0, T ; W^{1,6}(\Omega)\right)}+\left\|\partial_{t}\left(z_{n, \sigma_{3}}-\operatorname{tr}\left(\mathbb{T}_{n, \sigma_{3}}\right)\right)\right\|_{L^{2}\left(0, T ; L^{6}(\Omega)\right)} \leq C\left(L, \theta, n, E_{0, \sigma_{1}, \theta}, T\right) .
$$

Next, it follows from (7.16), via the diagonalisation process (6.21), for any $\beta>0$ with $2 \sigma_{3}<\beta<\sigma_{3}^{-1}$ that

$$
\begin{aligned}
\operatorname{tr}\left(\mathbb{P}-\beta \mathcal{G}_{\sigma_{3}}(\mathbb{P})\right)+d(\beta \log \beta-\beta) & =\sum_{j=1}^{d}\left[\left(\mathfrak{l}_{j}-\beta \mathcal{G}_{\sigma_{3}}\left(\mathfrak{l}_{j}\right)\right)+(\beta \log \beta-\beta)\right] \\
& \geq \sum_{j=1}^{d}\left[-\frac{\beta}{2 \sigma_{3}}\left[\mathfrak{l}_{j}\right]_{-}+\left(1-\frac{\beta}{2 \sigma_{3}}\right)\left[\mathfrak{l}_{j}\right]_{-}\right] \\
& \geq \frac{\beta}{2 \sigma_{3}} \sum_{j=1}^{d}\left|\left[\mathfrak{l}_{j}\right]_{-}\right| \geq \frac{\beta}{2 \sigma_{3}}\left(\sum_{j=1}^{d}\left[\mathfrak{l}_{j}\right]_{-}^{2}\right)^{\frac{1}{2}} \geq \frac{\beta}{2 \sigma_{3}}\left|[\mathbb{D}]_{-}\right|=\frac{\beta}{2 \sigma_{3}}\left|[\mathbb{P}]_{-}\right| .
\end{aligned}
$$

Hence, we have from $(7.30)_{1},(7.15)$ and $(7.41)$ that, for $\sigma_{3}<\min \left\{\theta, \frac{1}{2} \mathfrak{a}, \mathfrak{a}^{-1}, \tau_{\min },\left(\mathfrak{b}^{\star}\right)^{-1}\right\}$,

$$
\sup _{t \in(0, T]} \int_{\Omega}\left|\left[\mathbb{T}_{n, \sigma_{3}}\right]_{-}\right| \mathrm{d} x \leq C\left(E_{0, \sigma_{1}, \theta}, T\right) \sigma_{3} .
$$

Similarly, we note from (6.42), as $\mathbb{T}_{n, \sigma_{3}}$ is symmetric, (7.17) and (6.43) that, for $\sigma_{3}<\mathfrak{a}<\sigma_{3}^{-1}$,

$$
\begin{aligned}
\mathfrak{b} \int_{\Omega}\left[\left(1-\frac{z_{n, \sigma_{3}}}{\mathfrak{b}}\right)\right. & \left.-\mathcal{G}_{\sigma_{3}}\left(1-\frac{z_{n, \sigma_{3}}}{\mathfrak{b}}\right)\right] \mathrm{d} x \\
& =-\int_{\Omega}\left[\operatorname{tr}\left(\mathbb{T}_{n, \sigma_{3}}\right)+\mathfrak{b} \mathcal{G}_{\sigma_{3}}\left(1-\frac{z_{n, \sigma_{3}}}{\mathfrak{b}}\right)-\mathfrak{b}\right] \mathrm{d} x \\
& \leq-\int_{\Omega}\left[\mathfrak{a} \operatorname{tr}\left(\mathcal{G}_{\sigma_{3}}\left(\mathbb{T}_{n, \sigma_{3}}\right)\right)+\mathfrak{b} \mathcal{G}_{\sigma_{3}}\left(1-\frac{z_{n, \sigma_{3}}}{\mathfrak{b}}\right)-(\mathfrak{b}+d(\mathfrak{a} \log \mathfrak{a}-\mathfrak{a}))\right] \mathrm{d} x \\
& =\int_{\Omega}\left[-2 \Pi_{\sigma_{3}}^{(2)}\left(\mathbb{T}_{n, \sigma_{3}}, z_{n, \sigma_{3}}\right)+2 \mathfrak{b}+d(\mathfrak{a} \log \mathfrak{a}-\mathfrak{a})\right] \mathrm{d} x .
\end{aligned}
$$

Thus we have from $(7.30)_{1},(7.43)$ and a scalar version of $(7.41)$ that, for $\sigma_{3}<\sigma_{3, \min }:=\min \left\{\theta, \frac{1}{2}, \frac{1}{2} \mathfrak{a}, \mathfrak{a}^{-1}, \tau_{\min },\left(\mathfrak{b}^{\star}\right)^{-1}\right\}$,

$$
\sup _{t \in(0, T]} \int_{\Omega}\left|\left[1-\frac{z_{n, \sigma_{3}}}{\mathfrak{b}}\right]_{-}\right| \mathrm{d} x \leq C\left(E_{0, \sigma_{1}, \theta}, T\right) \sigma_{3} .
$$

After these preparatory considerations, we are now ready to pass to the limit $\sigma_{3} \rightarrow 0$ in the fourth level of approximation, so as to deduce the existence of solutions to the third level of approximation. This will be the subject of the next section.

\section{THE THIRD LEVEL OF APPROXIMATION}

This section is devoted to studying the limit of the solution sequence $\left(\varrho_{n, \sigma_{3}}, \mathbf{u}_{n, \sigma_{3}}, \mathbb{T}_{n, \sigma_{3}}, z_{n, \sigma_{3}}\right)$ as $\sigma_{3} \rightarrow 0$, and to showing that the resulting limit is a solution to the third level of approximation formulated in Section 6.4. We will also derive bounds on this solution limit that are uniform with respect to $n$, in preparation for passage to the limit $n \rightarrow \infty$ in the next section. 
8.1. Basic convergence results. The bounds in (7.22) and (7.26) with $T_{n, \sigma_{3}}$ replaced by $T$, the definition (7.18) and the bounds (7.29) and (7.37) imply the following weak convergence results for a subsequence, which will not be indicated throughout the paper, as $\sigma_{3} \rightarrow 0$ :

$$
\begin{aligned}
\mathbf{u}_{n, \sigma_{3}} \rightarrow \mathbf{u}_{n} & \text { weakly in } L^{2}\left(0, T ; W^{1,2}(\Omega)\right), \\
\varrho_{n, \sigma_{3}} \rightarrow \varrho_{n} & \text { weakly-* in } L^{\infty}\left(0, T ; L^{\Gamma}(\Omega)\right) \cap L^{\infty}\left(0, T ; W^{1,2}(\Omega)\right) \cap L^{2}\left(0, T ; W^{2,2}(\Omega)\right) \cap W^{1,2}\left(0, T ; L^{2}(\Omega)\right), \\
\mathbb{T}_{n, \sigma_{3}} \rightarrow \mathbb{T}_{n} & \text { weakly-* in } L^{\infty}\left(0, T ; W^{1,2}\left(\Omega ; \mathbb{R}^{d \times d}\right)\right) \cap L^{2}\left(0, T ; W^{2,2}\left(\Omega ; \mathbb{R}^{d \times d}\right)\right) \cap W^{1,2}\left(0, T ; L^{2}\left(\Omega ; \mathbb{R}^{d \times d}\right)\right), \\
z_{n, \sigma_{3}} \rightarrow z_{n} & \text { weakly-* in } L^{\infty}\left(0, T ; W^{1,2}(\Omega)\right) \cap L^{2}\left(0, T ; W^{2,2}(\Omega)\right) \cap W^{1,2}\left(0, T ; L^{2}(\Omega)\right) .
\end{aligned}
$$

Using the Aubin-Lions-Simon compactness theorem (cf. Lemma 5.3) we then deduce the following strong convergence results, as $\sigma_{3} \rightarrow 0$ :

$$
\begin{array}{rlrl}
\varrho_{n, \sigma_{3}} \rightarrow \varrho_{n} & & \text { strongly in } L^{2}\left(0, T ; W^{1, q}(\Omega)\right) \cap C\left([0, T] ; L^{q}(\Omega)\right), \\
\mathbb{T}_{n, \sigma_{3}} \rightarrow \mathbb{T}_{n} & \text { strongly in } L^{2}\left(0, T ; W^{1, q}\left(\Omega ; \mathbb{R}^{d \times d}\right)\right) \cap C\left([0, T] ; L^{q}\left(\Omega ; \mathbb{R}^{d \times d}\right)\right), \\
z_{n, \sigma_{3}} \rightarrow z_{n} & \text { strongly in } L^{2}\left(0, T ; W^{1, q}(\Omega)\right) \cap C\left([0, T] ; L^{q}(\Omega)\right),
\end{array}
$$

where $q \in[1, \infty)$ if $d=2$ and $q \in[1,6)$ if $d=3$. It follows from $(8.2)_{1}$ and $(7.29)_{2}$ that

$$
0 \leq \varrho_{n} \quad \text { a.e. in }(0, T] \times \Omega .
$$

In addition, the limit $\mathbb{T}_{n}$ is also real symmetric as $\mathbb{T}_{\sigma_{3}, n}$ is real symmetric for all $\sigma_{3} \in\left(0, \sigma_{3, \text { min }}\right)$, recall the line above (7.44), and all $n \in \mathbb{N}$. Moreover, we deduce from $(8.2)_{2,3},(7.42)$ and (7.44) that

$$
0 \leq \mathbb{T}_{n} \quad \text { and } \quad z_{n} \leq \mathfrak{b} \quad \text { a.e. in }(0, T] \times \Omega .
$$

As $\chi_{\sigma_{3}},(6.30)$, is globally Lipschitz with Lipschitz constant 1, it immediately follows from (8.4), (8.2) 2,3 and Lebesgue's dominated convergence theorem that

$$
\begin{aligned}
\chi_{\sigma_{3}}\left(\mathbb{T}_{n, \sigma_{3}}\right) & \rightarrow \mathbb{T}_{n} & & \text { strongly in } L^{2}\left(0, T ; W^{1, q}\left(\Omega ; \mathbb{R}^{d \times d}\right)\right) \cap C\left([0, T] ; L^{q}\right. \\
\chi_{\sigma_{3}}\left(1-\frac{z_{n, \sigma_{3}}}{\mathfrak{b}}\right) & \rightarrow 1-\frac{z_{n}}{\mathfrak{b}} & & \text { strongly in } L^{2}\left(0, T ; W^{1, q}(\Omega)\right) \cap C\left([0, T] ; L^{q}(\Omega)\right),
\end{aligned}
$$

as $\sigma_{3} \rightarrow 0$, where $q \in[1, \infty)$ if $d=2$ and $q \in[1,6)$ if $d=3$.

We deduce from (7.22) with $T_{n, \sigma_{3}}$ replaced by $T$ and Sobolev embedding that

$$
\left\|\varrho_{n, \sigma_{3}}^{\frac{\Gamma}{2}}\right\|_{L^{2}\left(0, T ; L^{6}(\Omega)\right)} \leq C\left\|\varrho_{n}^{\frac{\Gamma}{2}} \sigma_{3}\right\|_{L^{2}\left(0, T ; W^{1,2}(\Omega)\right)},
$$

and therefore $\left\|\varrho_{n, \sigma_{3}}^{\Gamma}\right\|_{L^{1}\left(0, T ; L^{3}(\Omega)\right)} \leq C\left(\sigma_{1}, \sigma_{2}, E_{0, \sigma_{1}, \theta}, T\right)$. Hence, by (7.22) again and interpolation between Lebesgue spaces, we deduce that

$$
\left\|\varrho_{n, \sigma_{3}}^{\Gamma}\right\|_{L^{\frac{4}{3}}\left(0, T ; L^{2}(\Omega)\right)} \leq\left\|\varrho_{n, \sigma_{3}}^{\Gamma}\right\|_{L^{1}\left(0, T ; L^{3}(\Omega)\right)}^{\frac{3}{4}}\left\|\varrho_{n, \sigma_{3}}^{\Gamma}\right\|_{L^{\infty}\left(0, T ; L^{1}(\Omega)\right)}^{\frac{1}{4}} \leq C\left(\sigma_{1}, \sigma_{2}, E_{0, \sigma_{1}, \theta}, T\right) .
$$

Together with $(8.2)_{1}$, we obtain, for example, the strong convergence result using the Vitali convergence theorem, as $\sigma_{3} \rightarrow 0:$

$$
\varrho_{n, \sigma_{3}} \rightarrow \varrho_{n} \quad \text { strongly in } L^{\beta}\left(0, T ; L^{\beta}(\Omega)\right),
$$

where $\beta \in\left[1, \frac{4 \Gamma}{3}\right)$. Hence, it follows that as $\sigma_{3} \rightarrow 0$ :

$$
\varrho_{n, \sigma_{3}}^{\gamma} \rightarrow \varrho_{n}^{\gamma}, \quad \varrho_{n, \sigma_{3}}^{\Gamma} \rightarrow \varrho_{n}^{\Gamma} \quad \text { strongly in } L^{1}\left(0, T ; L^{1}(\Omega)\right) .
$$

8.2. Bound on the trace and the positivity of the conformation tensor $\mathbb{T}_{n}$. Employing techniques from $[1,2]$, we will now improve on (8.4), by using the bound $(7.30)_{2}$, to show that $\mathbb{T}_{n}$ is, almost everywhere, a real symmetric positive definite matrix with $\operatorname{tr}\left(\mathbb{T}_{n}\right)<\mathfrak{b}$.

Lemma 8.1. We have that $z_{n}=\operatorname{tr}\left(\mathbb{T}_{n}\right)$ a.e. on $(0, T] \times \Omega$.

Proof. Multiplying (6.41) with any $\eta \in C^{\infty}(\bar{\Omega})$, integrating over $\Omega$, performing integration by parts, noting (6.40), and the boundary conditions (1.9), (1.10) for $\mathbf{u}_{n, \sigma_{3}}$ and $\mathbb{T}_{n, \sigma_{3}}$ we obtain for a.e. $t \in(0, T]$ that

$$
\begin{gathered}
\int_{\Omega} \partial_{t}\left(z_{n, \sigma_{3}}-\operatorname{tr}\left(\mathbb{T}_{n, \sigma_{3}}\right)\right) \eta \mathrm{d} x+\mathfrak{b} \int_{\Omega}\left[\chi_{\sigma_{3}}\left(1-\frac{z_{n, \sigma_{3}}}{\mathfrak{b}}\right)-\left(1-\frac{\operatorname{tr}\left(\chi_{\sigma_{3}}\left(\mathbb{T}_{n, \sigma_{3}}\right)\right)}{\mathfrak{b}}\right)\right] \mathbf{u}_{n, \sigma_{3}} \cdot \nabla_{x} \eta \mathrm{d} x \\
+\varepsilon \int_{\Omega} \nabla_{x}\left(z_{n, \sigma_{3}}-\operatorname{tr}\left(\mathbb{T}_{n, \sigma_{3}}\right)\right) \cdot \nabla_{x} \eta \mathrm{d} x=0 .
\end{gathered}
$$


We now multiply (8.10) by a test function $\psi \in C^{\infty}[0, T]$, integrate over $(0, T)$, pass to the limit $\sigma_{2} \rightarrow 0$ using the convergence results $(8.1)_{1,3,4}$ and (8.5), and since the resulting equality holds for all $\psi \in C^{\infty}([0, T])$ we deduce that

$$
\int_{\Omega} \partial_{t}\left(z_{n}-\operatorname{tr}\left(\mathbb{T}_{n}\right)\right) \eta \mathrm{d} x-\int_{\Omega}\left(z_{n}-\operatorname{tr}\left(\mathbb{T}_{n}\right)\right) \mathbf{u}_{n} \cdot \nabla_{x} \eta \mathrm{d} x+\varepsilon \int_{\Omega} \nabla_{x}\left(z_{n}-\operatorname{tr}\left(\mathbb{T}_{n}\right)\right) \cdot \nabla_{x} \eta \mathrm{d} x=0
$$

holds for a.e. $t \in(0, T]$. As $z_{n}(0, \cdot)=\operatorname{tr}\left(\mathbb{T}_{n}(0, \cdot)\right)$, uniqueness of this parabolic problem $(8.11)$ is easily established by noting the $\sigma_{3}$-uniform bound $(7.29)_{1}$. Hence, we have that $z_{n}=\operatorname{tr}\left(\mathbb{T}_{n}\right)$ a.e. on $(0, T] \times \Omega$.

Therefore, it follows from (8.4), (8.2) 2 and Lemma 8.1, as $L \geq \mathfrak{b}$, that

$$
\chi_{\sigma_{3}}^{L}\left(\mathbb{T}_{n, \sigma_{3}}\right) \rightarrow \mathbb{T}_{n} \quad \text { strongly in } L^{2}\left(0, T ; W^{1, q}\left(\Omega ; \mathbb{R}^{d \times d}\right)\right) \cap C\left([0, T] ; L^{q}\left(\Omega ; \mathbb{R}^{d \times d}\right)\right),
$$

as $\sigma_{3} \rightarrow 0$, where $q \in[1, \infty)$ if $d=2$ and $q \in[1,6)$ if $d=3$.

Similarly to (6.39), we introduce

$$
\mathbb{A}_{n, \sigma_{3}}^{L, \star \star}:=\left[\chi_{\sigma_{3}}\left(1-\frac{z_{n, \sigma_{3}}}{\mathfrak{b}}\right)\right]^{-1} \chi_{\sigma_{3}}^{L}\left(\mathbb{T}_{n, \sigma_{3}}\right)-\mathfrak{a} \mathbb{I} \quad \text { and } \quad \mathbb{A}_{n, \sigma_{3}}^{\star}:=\left[\chi_{\sigma_{3}}\left(1-\frac{z_{n, \sigma_{3}}}{\mathfrak{b}}\right)\right]^{-1} \chi_{\sigma_{3}}\left(\mathbb{T}_{n, \sigma_{3}}\right)-\mathfrak{a} \mathbb{I},
$$

where we recall that $\mathbb{T}_{n, \sigma_{3}}$ is symmetric. We note that

$$
\mathbb{A}_{n, \sigma_{3}}^{L, \star}=\mathbb{A}_{n, \sigma_{3}}^{L}+\mathbb{B}_{n, \sigma_{3}}^{L}, \quad \text { where } \quad \mathbb{B}_{n, \sigma_{3}}^{L}=\mathfrak{a}\left(\left[\chi_{\sigma_{3}}\left(\mathbb{T}_{n, \sigma_{3}}\right)\right]^{-1}-\left[\chi_{\sigma_{3}}^{L}\left(\mathbb{T}_{n, \sigma_{3}}\right)\right]^{-1}\right) \chi_{\sigma_{3}}^{L}\left(\mathbb{T}_{n, \sigma_{3}}\right) .
$$

Via a diagonalisation process, recall (6.21), we have, by noting (6.30), that

$$
\left|\mathbb{B}_{n, \sigma_{3}}^{L}\left[\chi_{\sigma_{3}}^{L}\left(\mathbb{T}_{n, \sigma_{3}}\right)\right]^{-\frac{1}{2}}\right|^{2}=\mathfrak{a}^{2} \sum_{j=1}^{d}\left(\left[\chi_{\sigma_{3}}\left(\mathfrak{l}_{j}\right)\right]^{-1}-\left[\chi_{\sigma_{3}}^{L}\left(\mathfrak{l}_{j}\right)\right]^{-1}\right)^{2} \chi_{\sigma_{3}}^{L}\left(\mathfrak{l}_{j}\right) \leq \mathfrak{a}^{2} d L^{-1},
$$

where $\left\{\mathfrak{l}_{j}\right\}_{j=1}^{d}$ are the eigenvalues of $\mathbb{T}_{n, \sigma_{3}}$. As $\chi_{\sigma_{3}}^{L}\left(\mathbb{T}_{n, \sigma_{3}}\right)$ is symmetric positive definite, we deduce from $(8.14),(7.30)_{2}$ and (8.15) that

(8.16)

$$
\begin{aligned}
\int_{0}^{T} \int_{\Omega} \operatorname{tr}\left(\left(\mathbb{A}_{n, \sigma_{3}}^{L, \star \star}\right)^{2}\left[\chi_{\sigma_{3}}^{L}\left(\mathbb{T}_{n, \sigma_{3}}\right)\right]^{-1}\right) \mathrm{d} x \mathrm{~d} t & =\int_{0}^{T} \int_{\Omega}\left|\left(\mathbb{A}_{n, \sigma_{3}}^{L}+\mathbb{B}_{n, \sigma_{3}}^{L}\right)\left[\chi_{\sigma_{3}}^{L}\left(\mathbb{T}_{n, \sigma_{3}}\right)\right]^{-\frac{1}{2}}\right|^{2} \mathrm{~d} x \mathrm{~d} t \\
& \leq 2 \int_{0}^{T} \int_{\Omega}\left[\left|\mathbb{A}_{n, \sigma_{3}}^{L}\left[\chi_{\sigma_{3}}^{L}\left(\mathbb{T}_{n, \sigma_{3}}\right)\right]^{-\frac{1}{2}}\right|^{2}+\left|\mathbb{B}_{n, \sigma_{3}}^{L}\left[\chi_{\sigma_{3}}^{L}\left(\mathbb{T}_{n, \sigma_{3}}\right)\right]^{-\frac{1}{2}}\right|^{2}\right] \mathrm{d} x \mathrm{~d} t \\
& \leq C\left(E_{0, \sigma_{1}, \theta}, T\right) .
\end{aligned}
$$

Hence, similarly to (7.31), we deduce from (8.16) and (6.30) that

$$
\left\|\mathbb{A}_{n, \sigma_{3}}^{L, \star}\right\|_{L^{2}\left(0, T ; L^{2}\left(\Omega, \mathbb{R}^{d \times d}\right)\right)}^{2} \leq C\left(L, E_{0, \sigma_{1}, \theta}, T\right) .
$$

Lemma 8.2. We have that

$$
z_{n}=\operatorname{tr}\left(\mathbb{T}_{n}\right)<\mathfrak{b} \quad \text { and } \quad \mathbb{T}_{n}>0 \quad \text { a.e. in }(0, T] \times \Omega .
$$

In addition, by recalling (1.7), we have the following convergence result for $\sigma_{3} \rightarrow 0$ :

$$
\mathbb{A}_{n, \sigma_{3}}^{L, \star}+\mathfrak{a} \mathbb{I}=\left[\chi_{\sigma_{3}}\left(1-\frac{z_{n, \sigma_{3}}}{\mathfrak{b}}\right)\right]^{-1} \chi_{\sigma_{3}}^{L}\left(\mathbb{T}_{n, \sigma_{3}}\right) \rightarrow \mathbb{A}\left(\mathbb{T}_{n}\right)+\mathfrak{a} \mathbb{I} \quad \text { strongly in } L^{r}\left(0, T ; L^{r}\left(\Omega ; \mathbb{R}^{d \times d}\right)\right),
$$

for any $r \in[1,2)$.

Proof. We know already from (8.4) that $z_{n} \leq \mathfrak{b}$ a.e. in $(0, T] \times \Omega$. Assume that $z_{n}=\mathfrak{b}$ a.e. in $D_{n}^{b} \subset(0, T] \times \Omega$, we have that

$$
\mathfrak{b}\left|D_{n}^{b}\right|=\int_{D_{n}^{b}} \operatorname{tr}\left(\mathbb{T}_{n}\right) \mathrm{d} x \mathrm{~d} t=\int_{D_{n}^{b}} \operatorname{tr}\left(\mathbb{T}_{n}-\chi_{\sigma_{3}}^{L}\left(\mathbb{T}_{n, \sigma_{3}}\right)\right) \mathrm{d} x \mathrm{~d} t+\int_{D_{n}^{b}} \operatorname{tr}\left(\chi_{\sigma_{3}}^{L}\left(\mathbb{T}_{n, \sigma_{3}}\right)\right) \mathrm{d} x \mathrm{~d} t=: S_{1}+S_{2} .
$$

It follows from (8.12) that $S_{1} \rightarrow 0$ as $\sigma_{3} \rightarrow 0$. A simple rearrangement, by recalling (8.13), yields that

$$
\begin{aligned}
S_{2} & =\int_{D_{n}^{b}}\left[\operatorname{tr}\left(\left[\chi_{\sigma_{3}}\left(1-\frac{z_{n, \sigma_{3}}}{\mathfrak{b}}\right)\right]^{-1} \chi_{\sigma_{3}}^{L}\left(\mathbb{T}_{n, \sigma_{3}}\right)-\mathfrak{a} \mathbb{I}\right)+d \mathfrak{a}\right] \chi_{\sigma_{3}}\left(1-\frac{z_{n, \sigma_{3}}}{\mathfrak{b}}\right) \mathrm{d} x \mathrm{~d} t \\
& =\int_{D_{n}^{b}}\left[\operatorname{tr}\left(\mathbb{A}_{n, \sigma_{3}}^{L, \star}\right)+d \mathfrak{a}\right] \chi_{\sigma_{3}}\left(1-\frac{z_{n, \sigma_{3}}}{\mathfrak{b}}\right) \mathrm{d} x \mathrm{~d} t .
\end{aligned}
$$

Next, we observe that for any symmetric $\mathbb{P} \in \mathbb{R}^{d \times d}$ one has

$$
[\operatorname{tr}(\mathbb{P})]^{2} \leq d \operatorname{tr}\left(\mathbb{P}^{2}\right)=d|\mathbb{P}|^{2} .
$$


It then follows from $(8.21),(8.22)$ and $(8.17)$ that

$$
S_{2}^{2} \leq C\left(L, E_{0, \sigma_{1}, \theta}, T\right) \int_{D_{n}^{b}}\left[\chi_{\sigma_{3}}\left(1-\frac{z_{n, \sigma_{3}}}{\mathfrak{b}}\right)\right]^{2} \mathrm{~d} x \mathrm{~d} t .
$$

As $z_{n}=\mathfrak{b}$ a.e. in $D_{n}^{b}$, we have that

$$
S_{2} \leq C\left(L, E_{0, \sigma_{1}, \theta}, T\right)\left(\int_{D_{n}^{b}}\left|\chi_{\sigma_{3}}\left(1-\frac{z_{n, \sigma_{3}}}{\mathfrak{b}}\right)-\left(1-\frac{z_{n}}{\mathfrak{b}}\right)\right|^{2} \mathrm{~d} x \mathrm{~d} t\right)^{\frac{1}{2}} .
$$

It follows from (8.5) $)_{2}$ that $S_{2} \rightarrow 0$ as $\sigma_{3} \rightarrow 0$. Hence, we deduce from (8.20) that $\left|D_{n}^{b}\right|=0$, and so the first desired result in (8.18) holds by recalling Lemma 8.1.

We now establish the second result in (8.18), which improves on the first result (8.4). If the symmetric matrix function $\mathbb{T}_{n}$ were not positive definite a.e. in $D_{n}^{0} \subset(0, T] \times \Omega$, then there would exist a $\mathbf{q} \in L^{\infty}\left((0, T] \times \Omega ; \mathbb{R}^{d}\right)$ such that

$$
\mathbb{T}_{n} \mathbf{q}=\mathbf{0} \text { a.e. in }(0, T] \times \Omega \quad \text { with } \quad|\mathbf{q}|=1 \text { a.e. in } D_{n}^{0} \text { and } \mathbf{q}=\mathbf{0} \text { a.e. in }((0, T] \times \Omega) \backslash D_{n}^{0} .
$$

Recalling (8.13), we would have that

$$
\mathfrak{a}\left|D_{n}^{0}\right|=\mathfrak{a} \int_{0}^{T} \int_{\Omega}|\mathbf{q}| \mathrm{d} x \mathrm{~d} t \leq \int_{0}^{T} \int_{\Omega}\left|\mathbb{A}_{n, \sigma_{3}}^{L, \star} \mathbf{q}\right| \mathrm{d} x \mathrm{~d} t+\int_{0}^{T} \int_{\Omega}\left|\left(\mathbb{A}_{n, \sigma_{3}}^{L, \star}+\mathfrak{a} \mathbb{I}\right) \mathbf{q}\right| \mathrm{d} x \mathrm{~d} t:=S_{3}+S_{4} .
$$

As $\chi_{\sigma_{3}}^{L}\left(\mathbb{T}_{n, \sigma_{3}}\right)$ is symmetric positive definite, we have from (8.22) that

$$
\left|\mathbb{A}_{n, \sigma_{3}}^{L, \star} \mathbf{q}\right| \leq\left|\mathbb{A}_{n, \sigma_{3}}^{L, \star}\left[\chi_{\sigma_{3}}\left(\mathbb{T}_{n, \sigma_{3}}\right)\right]^{-\frac{1}{2}}\right|\left|\left[\chi_{\sigma_{3}}\left(\mathbb{T}_{n, \sigma_{3}}\right)\right]^{\frac{1}{2}} \mathbf{q}\right|=\left[\operatorname{tr}\left(\left(\mathbb{A}_{n, \sigma_{3}}^{L, \star}\right)^{2}\left[\chi_{\sigma_{3}}^{L}\left(\mathbb{T}_{n, \sigma_{3}}\right)\right]^{-1}\right)\left(\chi_{\sigma_{3}}^{L}\left(\mathbb{T}_{n, \sigma_{3}}\right):\left(\mathbf{q} \mathbf{q}^{\mathrm{T}}\right)\right)\right]^{\frac{1}{2}} .
$$

Combining (8.26) and (8.27), and noting (8.16), yields that

$$
S_{3} \leq C\left(L, E_{0, \sigma_{1}, \theta}, T\right)\left(\int_{0}^{T} \int_{\Omega} \chi_{\sigma_{3}}^{L}\left(\mathbb{T}_{n, \sigma_{3}}\right):\left(\mathbf{q q}^{\mathrm{T}}\right)\right)^{\frac{1}{2}} .
$$

It follows from (8.12) and (8.25) that $S_{3} \rightarrow 0$ as $\sigma_{3} \rightarrow 0$. Similarly to (8.27), on recalling (8.13) and (8.22), we have that

$$
S_{4} \leq d^{\frac{1}{4}}\left\|\mathbb{A}_{n, \sigma_{3}}^{L, \star}+\mathfrak{a} \mathbb{I}\right\|_{L^{1}\left(0, T ; L^{1}\left(\Omega ; \mathbb{R}^{d \times d}\right)\right)}^{\frac{1}{2}}\left(\int_{0}^{T} \int_{\Omega}\left[\chi_{\sigma_{3}}\left(1-\frac{z_{n, \sigma_{3}}}{\mathfrak{b}}\right)\right]^{-1} \chi_{\sigma_{3}}^{L}\left(\mathbb{T}_{n, \sigma_{3}}\right):\left(\mathbf{q}^{\mathrm{T}}\right) \mathrm{d} x \mathrm{~d} t\right)^{\frac{1}{2}} .
$$

It follows from (8.17) that

$$
\left\|\mathbb{A}_{n, \sigma_{3}}^{L, \star}+\mathfrak{a} \mathbb{I}\right\|_{L^{2}\left(0, T ; L^{2}\left(\Omega ; \mathbb{R}^{d \times d}\right)\right)} \leq C\left(L, E_{0, \sigma_{1}, \theta}, T\right) .
$$

By recalling (1.7), we will now show that

$$
\mathbb{A}_{n, \sigma_{3}}^{L, \star}+\mathfrak{a} \mathbb{I}=\left[\chi_{\sigma_{3}}\left(1-\frac{z_{n, \sigma_{3}}}{\mathfrak{b}}\right)\right]^{-1} \chi_{\sigma_{3}}^{L}\left(\mathbb{T}_{n, \sigma_{3}}\right) \rightarrow \mathbb{A}\left(\mathbb{T}_{n}\right)+\mathfrak{a} \mathbb{I} \quad \text { strongly in } L^{r}\left(0, T ; L^{r}\left(\Omega ; \mathbb{R}^{d \times d}\right)\right),
$$

for any $r \in[1,2)$ as $\sigma_{3} \rightarrow 0$, upon possibly extracting a subsequence. It immediately follows from $(8.5)_{2}$ and (8.12) that $\mathbb{A}_{n, \sigma_{3}}^{L, \star}+\mathfrak{a} \mathbb{I} \rightarrow \mathbb{A}\left(\mathbb{T}_{n}\right)+\mathfrak{a} \mathbb{I}$ a.e. in $(0, T] \times \Omega$, as $\sigma_{3} \rightarrow 0$, since we have already established the first result in (8.18). Hence, the desired result (8.19) follows by noting (8.30) and the Vitali convergence theorem. Finally, it follows from (8.29), (8.30), (8.19) and (8.25) that $S_{4} \rightarrow 0$ as $\sigma_{3} \rightarrow 0$. Therefore, (8.26) yields the second result in (8.18).

We now prove convergence results that are required in passing to the limit $\sigma_{3} \rightarrow 0$ in (6.37), (6.38) and (6.33).

Lemma 8.3. Similarly to (8.19), we have the following convergence results for $\sigma_{3} \rightarrow 0$ :

$$
\mathbb{A}_{n, \sigma_{3}}^{L} \rightarrow \mathbb{A}\left(\mathbb{T}_{n}\right) \quad \text { strongly in } L^{r}\left(0, T ; L^{r}\left(\Omega ; \mathbb{R}^{d \times d}\right)\right),
$$

for any $r \in[1,2)$; and

$$
\mathbb{A}_{n, \sigma_{3}}^{\star}+\mathfrak{a} \mathbb{I}=\left[\chi_{\sigma_{3}}\left(1-\frac{z_{n, \sigma_{3}}}{\mathfrak{b}}\right)\right]^{-1} \chi_{\sigma_{3}}\left(\mathbb{T}_{n, \sigma_{3}}\right) \rightarrow \mathbb{A}\left(\mathbb{T}_{n}\right)+\mathfrak{a} \mathbb{I} \quad \text { strongly in } L^{r}\left(0, T ; L^{r}\left(\Omega ; \mathbb{R}^{d \times d}\right)\right),
$$

for any $r \in\left[1, \frac{8}{5}\right)$. 
Proof. Similarly to (8.30) and (8.31), we deduce from (7.31), (6.39), (8.5), (8.12), (8.18), (1.7) and the Vitali convergence theorem that (8.32) holds by possibly extracting a subsequence.

We obtain from (6.39) and (8.13) that

$$
\mathbb{A}_{n, \sigma_{3}}^{\star}=\mathbb{A}_{n, \sigma_{3}}^{L}\left[\chi_{\sigma_{3}}^{L}\left(\mathbb{T}_{n, \sigma_{3}}\right)\right]^{-1} \chi_{\sigma_{3}}\left(\mathbb{T}_{n, \sigma_{3}}\right) .
$$

Therefore, we deduce from (8.34) and (6.30) that

$$
\left|\mathbb{A}_{n, \sigma_{3}}^{\star}\right| \leq\left|\mathbb{A}_{n, \sigma_{3}}^{L}\right|\left|\left[\chi_{\sigma_{3}}^{L}\left(\mathbb{T}_{n, \sigma_{3}}\right)\right]^{-1} \chi_{\sigma_{3}}\left(\mathbb{T}_{n, \sigma_{3}}\right)\right| \leq\left|\mathbb{A}_{n, \sigma_{3}}^{L}\right|\left(1+L^{-1}\left|\mathbb{T}_{n, \sigma_{3}}\right|\right) .
$$

It follows from interpolation of Lebesgue spaces, Sobolev embedding and (7.38) that

$$
\left\|\mathbb{T}_{n, \sigma_{3}}\right\|_{L^{8}\left(0, T ; L^{8}\left(\Omega ; \mathbb{R}^{d \times d}\right)\right)} \leq\left\|\mathbb{T}_{n, \sigma_{3}}\right\|_{L^{\infty}\left(0, T ; L^{6}\left(\Omega ; \mathbb{R}^{d \times d}\right)\right)}^{\frac{3}{4}}\left\|\mathbb{T}_{n, \sigma_{3}}\right\|_{L^{2}\left(0, T ; L^{\infty}\left(\Omega ; \mathbb{R}^{d \times d}\right)\right)}^{\frac{1}{4}} \leq C\left(L, \theta, n, E_{0, \sigma_{1}, \theta}, T\right) .
$$

On noting that $\frac{5}{8}=\frac{1}{2}+\frac{1}{8}$, the bounds (8.35), (7.31) and (8.36) yield that

$$
\begin{aligned}
& \left\|\mathbb{A}_{n, \sigma_{3}}^{\star}\right\|_{L^{\frac{8}{5}\left(0, T ; L^{\frac{8}{5}}\left(\Omega ; \mathbb{R}^{d \times d}\right)\right)}} \leq C(L)\left\|\mathbb{A}_{n, \sigma_{3}}^{L}\right\|_{L^{2}\left(0, T ; L^{2}\left(\Omega ; \mathbb{R}^{d \times d}\right)\right)}\left(1+\left\|\mathbb{T}_{n, \sigma_{3}}\right\|_{L^{8}\left(0, T ; L^{8}\left(\Omega ; \mathbb{R}^{d \times d}\right)\right)}\right) \\
& \leq C\left(L, \theta, n, E_{0, \sigma_{1}, \theta}, T\right) .
\end{aligned}
$$

Similarly to (8.30) and (8.31), we deduce from (8.37), (8.13), (8.5), (8.18), (1.7) and the Vitali convergence theorem that (8.33) holds by possibly extracting a subsequence.

We now prove convergence of the polymeric pressure term $\Pi_{\sigma_{3}}^{(1)}\left(\mathbb{T}_{n, \sigma_{3}}, z_{n, \sigma_{3}}\right)$ as $\sigma_{3} \rightarrow 0$.

Lemma 8.4. We have the following convergence results as $\sigma_{3} \rightarrow 0$ :

$$
\begin{aligned}
\nabla_{x} \operatorname{tr}\left(\log \chi_{\sigma_{3}}\left(\mathbb{T}_{n, \sigma_{3}}\right)\right) & \rightarrow \nabla_{x} \operatorname{tr}\left(\log \mathbb{T}_{n}\right) & & \text { weakly in } L^{2}\left(0, T ; L^{2}(\Omega)\right), \\
\nabla_{x} \log \left(\chi_{\sigma_{3}}\left(1-\frac{z_{n, \sigma_{3}}}{\mathfrak{b}}\right)\right) & \rightarrow \nabla_{x} \log \left(1-\frac{\operatorname{tr}\left(\mathbb{T}_{n}\right)}{\mathfrak{b}}\right) & & \text { weakly in } L^{2}\left(0, T ; L^{2}(\Omega)\right), \\
(I-f) \Pi_{\sigma_{3}}^{(1)}\left(\mathbb{T}_{n, \sigma_{3}}, z_{n, \sigma_{3}}\right) & \rightarrow(I-f) \Pi\left(\mathbb{T}_{n}\right) & & \text { strongly in } L^{r}\left(0, T ; L^{r}(\Omega)\right),
\end{aligned}
$$

for any $r \in\left[1, \frac{8}{5}\right)$.

Proof. The desired results $(8.38)_{1,2}$ follow immediately from (7.22), (8.5) and (8.18).

It follows from $(7.40),(8.2)_{2,3}$, Lemma 5.3 and Sobolev embedding that

$$
z_{n, \sigma_{3}}-\operatorname{tr}\left(\mathbb{T}_{n, \sigma_{3}}\right) \rightarrow 0 \quad \text { strongly in } C([0, T] ; C(\bar{\Omega})) .
$$

As a consequence of (8.39), we have that there exists a $\sigma_{3, b}<\sigma_{3, \min }$, recall the line above (7.44), such that, for all $\sigma_{3} \in\left(0, \sigma_{3, b}\right]$,

$$
\left\|z_{n, \sigma_{3}}-\operatorname{tr}\left(\mathbb{T}_{n, \sigma_{3}}\right)\right\|_{C([0, T] ; C(\bar{\Omega}))} \leq \frac{\mathfrak{b}}{4}
$$

We define

$$
\left(\Omega_{T}\right)_{b, \sigma_{3}}:=\left\{(t, x) \in \Omega_{T}: z_{n, \sigma_{3}} \leq \frac{\mathfrak{b}}{2}\right\},
$$

and note that we have from (8.40) and (8.41) that, for all $\sigma_{3} \in\left(0, \sigma_{3, b}\right]$,

$$
\operatorname{tr}\left(\mathbb{T}_{n, \sigma_{3}}\right) \geq \frac{\mathfrak{b}}{4} \quad \text { on } \Omega_{T} \backslash\left(\Omega_{T}\right)_{b, \sigma_{3}} .
$$

Using (8.41), we have for all $\sigma_{3} \in\left(0, \sigma_{3, b}\right]$ that

$$
\begin{aligned}
& \left\|\left[\chi_{\sigma_{3}}\left(1-\frac{z_{n, \sigma_{3}}}{\mathfrak{b}}\right)\right]^{-1}\right\|_{L^{\frac{8}{5}}\left(0, T ; L^{\frac{8}{5}}(\Omega)\right)} \\
& \quad \leq\left\|\left[\chi_{\sigma_{3}}\left(1-\frac{z_{n, \sigma_{3}}}{\mathfrak{b}}\right)\right]^{-1}\right\|_{L^{\frac{8}{5}\left(\left(\Omega_{T}\right)_{b, \sigma_{3}}\right)}}+\left\|\left[\chi_{\sigma_{3}}\left(1-\frac{z_{n, \sigma_{3}}}{\mathfrak{b}}\right)\right]^{-1}\right\|_{L^{\frac{8}{5}}\left(\Omega_{T} \backslash\left(\Omega_{T}\right)_{b, \sigma_{3}}\right)}=: S_{1}+S_{2} .
\end{aligned}
$$

It follows from (8.41) and the monotonicity of $\chi_{\sigma_{3}}(\cdot)$, recall (6.30), that, for all $\sigma_{3} \in\left(0, \sigma_{3, b}\right]$,

$$
S_{1} \leq\left\|\left[\chi_{\sigma_{3}}\left(\frac{1}{2}\right)\right]^{-1}\right\|_{L^{\frac{8}{5}\left(\left(\Omega_{T}\right)_{b, \sigma_{3}}\right)}} \leq 2\left|\Omega_{T}\right|^{\frac{5}{8}} .
$$


It follows from (8.13), (8.22), (8.37) and (8.42) that, for all $\sigma_{3} \in\left(0, \sigma_{3, b}\right]$,

$$
\begin{aligned}
S_{2} & =\left\|\left[\chi_{\sigma_{3}}\left(1-\frac{z_{n, \sigma_{3}}}{\mathfrak{b}}\right)\right]^{-1} \operatorname{tr}\left(\chi_{\sigma_{3}}\left(\mathbb{T}_{n, \sigma_{3}}\right)\right)\left[\operatorname{tr}\left(\chi_{\sigma_{3}}\left(\mathbb{T}_{n, \sigma_{3}}\right)\right)\right]^{-1}\right\|_{L^{\frac{8}{5}}\left(\Omega_{T} \backslash\left(\Omega_{T}\right)_{b, \sigma_{3}}\right)} \\
& \leq\left\|\left[\chi_{\sigma_{3}}\left(1-\frac{z_{n, \sigma_{3}}}{\mathfrak{b}}\right)\right]^{-1} \operatorname{tr}\left(\chi_{\sigma_{3}}\left(\mathbb{T}_{n, \sigma_{3}}\right)\right)\right\|_{L^{\frac{8}{5}\left(\Omega_{T} \backslash\left(\Omega_{T}\right)_{b, \sigma_{3}}\right)}}\left\|\left[\operatorname{tr}\left(\chi_{\sigma_{3}}\left(\mathbb{T}_{n, \sigma_{3}}\right)\right)\right]^{-1}\right\|_{L^{\infty}\left(\Omega_{T} \backslash\left(\Omega_{T}\right)_{b, \sigma_{3}}\right)} \\
& \leq C\left(L, \theta, n, E_{0, \sigma_{1}, \theta}, T\right),
\end{aligned}
$$

since $\operatorname{tr}\left(\chi_{\sigma_{3}}\left(\mathbb{T}_{n, \sigma_{3}}\right)\right) \geq \min \left\{\operatorname{tr}\left(\mathbb{T}_{n, \sigma_{3}}\right), \sigma_{3}^{-1}\right\} \geq \min \left\{\frac{\mathfrak{b}}{4}, \sigma_{3}^{-1}\right\}$ a.e. on $\Omega_{T} \backslash\left(\Omega_{T}\right)_{b, \sigma_{3}}$. Combining (8.43)-(8.45), the definition of $A_{n, \sigma_{3}}^{\star}$ in (8.13), (8.22) and (8.37), we have for all $\sigma_{3} \in\left(0, \sigma_{3, b}\right]$ that

$$
\left\|\left[\chi_{\sigma_{3}}\left(1-\frac{z_{n, \sigma_{3}}}{\mathfrak{b}}\right)\right]^{-1}\left(1-\frac{\operatorname{tr}\left(\chi_{\sigma_{3}}\left(\mathbb{T}_{n, \sigma_{3}}\right)\right)}{\mathfrak{b}}\right)\right\|_{L^{\frac{8}{5}\left(0, T ; L^{\frac{8}{5}}(\Omega)\right)}} \leq C\left(L, \theta, n, E_{0, \sigma_{1}, \theta}, T\right) .
$$

It follows from (6.35), the symmetry of $\mathbb{T}_{n, \sigma_{3}},(7.22)$ with $T_{n, \sigma_{3}}$ replaced with $T$, a Poincaré inequality and (8.46) that

$$
\begin{aligned}
\|(I-f) & \Pi_{\sigma_{3}}^{(1)}\left(\mathbb{T}_{n, \sigma_{3}}, z_{n, \sigma_{3}}\right) \|_{L^{\frac{8}{5}}\left(0, T ; L^{\frac{8}{5}}(\Omega)\right)} \\
\leq & \frac{1}{2}\left\|(I-f)\left[\mathfrak{a} \operatorname{tr}\left(\log \chi_{\sigma_{3}}\left(\mathbb{T}_{n, \sigma_{3}}\right)\right)+\mathfrak{b} \log \left(\chi_{\sigma_{3}}\left(1-\frac{z_{n, \sigma_{3}}}{\mathfrak{b}}\right)\right)\right]\right\|_{L^{\frac{8}{5}}\left(0, T ; L^{\frac{8}{5}}(\Omega)\right)} \\
\quad & \quad+\frac{\mathfrak{b}}{2}\left\|(I-f)\left[\chi_{\sigma_{3}}\left(1-\frac{z_{n, \sigma_{3}}}{\mathfrak{b}}\right)\right]^{-1}\left(1-\frac{\operatorname{tr}\left(\chi_{\sigma_{3}}\left(\mathbb{T}_{n, \sigma_{3}}\right)\right)}{\mathfrak{b}}\right)\right\|_{L^{\frac{8}{5}}\left(0, T ; L^{\frac{8}{5}}(\Omega)\right)} \\
\leq & C\left(L, \theta, n, E_{0, \sigma_{1}, \theta}, T\right) .
\end{aligned}
$$

Similarly to (8.33), we deduce from (8.47), by noting the Vitali convergence theorem, (8.5) and (8.18), the desired result $(8.38)_{3}$.

8.3. Convergence of $\mathbf{u}_{n, \sigma_{3}}$. Our aim in this section is to show that the function $t \in(0, T) \mapsto \mathbf{u}_{n, \sigma_{3}}(\cdot, t) \in X_{n}$ is weakly differentiable with respect to $t$ and that

$$
\left\|\partial_{t} \mathbf{u}_{n, \sigma_{3}}\right\|_{L^{\frac{8}{5}}\left(0, T ; L^{2}\left(\Omega ; \mathbb{R}^{d}\right)\right)} \leq C\left(\sigma_{2}, L, \theta, n, E_{0, \sigma_{1}, \theta}, T\right) .
$$

With the uniform bound (8.48) in hand, the continuous embedding $C^{2, \beta}\left(\bar{\Omega} ; \mathbb{R}^{d}\right) \hookrightarrow W^{2+\beta, 2}\left(\Omega ; \mathbb{R}^{d}\right)$, the compact embedding $W^{2+\beta, 2}\left(\Omega ; \mathbb{R}^{d}\right) \hookrightarrow \hookrightarrow W^{2,2}\left(\Omega ; \mathbb{R}^{d}\right)$, (7.29), and the Aubin-Lions-Simon compactness theorem, Lemma 5.3, yield, as $\sigma_{3} \rightarrow 0$, that:

$$
\mathbf{u}_{n, \sigma_{3}} \rightarrow \mathbf{u}_{n} \quad \text { strongly in } C\left([0, T] ; W^{2,2}\left(\Omega ; \mathbb{R}^{d}\right)\right), \quad \text { weakly in } W^{1, \frac{8}{5}}\left(0, T ; L^{2}\left(\Omega ; \mathbb{R}^{d}\right)\right) .
$$

In order to show (8.48), we fix a function $\varphi_{n} \in C_{0}^{1}\left((0, T) ; X_{n}\right)$, with support $\operatorname{supp} \varphi_{n} \subseteq\left[t_{*}, t_{* *}\right] \subset(0, T)$, and let $\Delta t>0$ be such that $\Delta t<\min \left\{t_{*}, T-t_{* *}\right\}$. We shall use the following notations for the forward and backward difference quotients of a function $v$ defined on $[0, T]$, which takes values in a certain linear space:

$$
\delta_{t} v(t):=\frac{v(t+\Delta t)-v(t)}{\Delta t}, \quad t \in[0, T-\Delta t], \quad \bar{\delta}_{t} v(t):=\frac{v(t)-v(t-\Delta t)}{\Delta t}, \quad t \in[\Delta t, T] .
$$

Clearly,

$$
\begin{aligned}
\int_{0}^{T} & \int_{\Omega} \varrho_{n, \sigma_{3}} \delta_{t} \mathbf{u}_{n, \sigma_{3}} \cdot \boldsymbol{\varphi}_{n} \mathrm{~d} x \mathrm{~d} t=-\int_{\Delta t}^{T} \int_{\Omega} \mathbf{u}_{n, \sigma_{3}} \cdot \bar{\delta}_{t}\left(\varrho_{n, \sigma_{3}} \boldsymbol{\varphi}_{n}\right) \mathrm{d} x \mathrm{~d} t \\
& =-\int_{\Delta t}^{T} \int_{\Omega} \mathbf{u}_{n, \sigma_{3}} \cdot\left(\varrho_{n, \sigma_{3}} \bar{\delta}_{t} \boldsymbol{\varphi}_{n}\right) \mathrm{d} x \mathrm{~d} t-\int_{\Delta t}^{T} \int_{\Omega} \mathbf{u}_{n, \sigma_{3}} \cdot\left(\boldsymbol{\varphi}_{n}(\cdot-\Delta t) \bar{\delta}_{t} \varrho_{n, \sigma_{3}}\right) \mathrm{d} x \mathrm{~d} t=: \mathrm{T}_{1}+\mathrm{T}_{2} .
\end{aligned}
$$

It remains to bound $T_{1}$ and $T_{2}$ in order to deduce (8.48). We begin by considering $T_{1}$. Let

$$
\boldsymbol{\psi}_{n}(\cdot, t):=\left\{\begin{array}{cl}
0 & \text { if } t \leq \Delta t \\
\int_{\Delta t}^{t} \bar{\delta}_{t} \boldsymbol{\varphi}_{n}(\cdot, s) \mathrm{d} s & \text { if } t \geq \Delta t .
\end{array}\right.
$$

A simple calculation reveals that $\boldsymbol{\psi}_{n}(\cdot, T)=\mathbf{0}$, whereby $\boldsymbol{\psi}_{n} \in C_{0}^{0,1}\left([0, T] ; X_{n}\right)$, and $\partial_{t} \boldsymbol{\psi}_{n}(\cdot, t)=\bar{\delta}_{t} \boldsymbol{\varphi}_{n}(\cdot, t)$ for all $t \in(-\infty, \infty)$, with both functions understood to be extended from their respective supports, contained in $[0, T]$, to $(-\infty, \infty)$ by 0 . Hence, and by partial integration with respect to $t$,

$$
\left|\mathrm{T}_{1}\right|=\left|\int_{\Delta t}^{T} \int_{\Omega} \partial_{t}\left(\varrho_{n, \sigma_{3}} \mathbf{u}_{n, \sigma_{3}}\right) \cdot \boldsymbol{\psi}_{n} \mathrm{~d} x \mathrm{~d} t\right|=\left|\int_{0}^{T} \int_{\Omega} \partial_{t}\left(\varrho_{n, \sigma_{3}} \mathbf{u}_{n, \sigma_{3}}\right) \cdot \boldsymbol{\psi}_{n} \mathrm{~d} x \mathrm{~d} t\right| .
$$


We then deduce from (6.33), (6.17), (1.4) and the bounds $(7.29)_{1,2},(8.46)$ and (8.47) that

$$
\left|\mathrm{T}_{1}\right| \leq C\left(\sigma_{2}, L, \theta, n, E_{0, \sigma_{1}, \theta}, T\right)\left\|\boldsymbol{\psi}_{n}\right\|_{L^{\frac{8}{3}}\left(0, T ; L^{2}\left(\Omega ; \mathbb{R}^{d}\right)\right)} .
$$

Our objective is now to show that $\left\|\boldsymbol{\psi}_{n}\right\|_{L^{\frac{8}{3}}\left(0, T ; L^{2}\left(\Omega ; \mathbb{R}^{d}\right)\right)} \leq\left\|\boldsymbol{\varphi}_{n}\right\|_{L^{\frac{8}{3}}\left(0, T ; L^{2}\left(\Omega ; \mathbb{R}^{d}\right)\right)}$, which will then result in our final bound on $\mathrm{T}_{1}$. To do so, we note that, for $t \geq \Delta t$,

$$
\boldsymbol{\psi}_{n}(\cdot, t)=\frac{1}{\Delta t} \int_{t-\Delta t}^{t} \boldsymbol{\varphi}_{n}(\cdot, s) \mathrm{d} s=\left(\chi_{\Delta t} *_{t} \boldsymbol{\varphi}_{n}\right)(\cdot, t),
$$

where $\chi_{\Delta t}(t):=\frac{1}{\Delta t} \chi_{[0, \Delta t]}$, with $\chi_{[0, \Delta t]}$ signifying the characteristic function of the interval $[0, \Delta t]$. For $t \leq \Delta t$ the equalities in (8.52) continue to hold, because $\boldsymbol{\psi}_{n}(\cdot, t)$, the middle term, and the convolution $\left(\chi_{\Delta t} *_{t} \boldsymbol{\varphi}_{n}\right)(\cdot, t)$ with respect to the independent variable $t$, are all equal to $\mathbf{0}$ for $t \leq \Delta t$. By Minkowski's integral inequality, therefore,

$$
\left\|\boldsymbol{\psi}_{n}(\cdot, t)\right\|_{L^{2}\left(\Omega ; \mathbb{R}^{d}\right)} \leq \frac{1}{\Delta t} \int_{t-\Delta t}^{t}\left\|\boldsymbol{\varphi}_{n}(\cdot, s)\right\|_{L^{2}\left(\Omega ; \mathbb{R}^{d}\right)} \mathrm{d} s=\chi_{\Delta t}(t) *_{t}\left\|\boldsymbol{\varphi}_{n}(\cdot, t)\right\|_{L^{2}\left(\Omega ; \mathbb{R}^{d}\right)} \quad \forall t \in \mathbb{R} .
$$

Hence and noting that $\left\|\chi_{\Delta t}\right\|_{L^{1}(\mathbb{R})}=1$, Young's convolution inequality yields that

$$
\left\|\boldsymbol{\psi}_{n}\right\|_{L^{\frac{8}{3}}\left(0, T ; L^{2}\left(\Omega ; \mathbb{R}^{d}\right)\right)}=\|\| \boldsymbol{\psi}_{n}\left\|_{L^{2}\left(\Omega ; \mathbb{R}^{d}\right)}\right\|_{L^{\frac{8}{3}}(0, T)}=\|\| \boldsymbol{\psi}_{n}\left\|_{L^{2}\left(\Omega ; \mathbb{R}^{d}\right)}\right\|_{L^{\frac{8}{3}(\mathbb{R})}} \leq\|\| \boldsymbol{\varphi}_{n}\left\|_{L^{2}\left(\Omega ; \mathbb{R}^{d}\right)}\right\|_{L^{\frac{8}{3}(\mathbb{R})}}=\left\|\boldsymbol{\varphi}_{n}\right\|_{L^{\frac{8}{3}}\left(0, T ; L^{2}\left(\Omega ; \mathbb{R}^{d}\right)\right)} \text {. }
$$

Substituting this into (8.51) it follows that

$$
\left|\mathrm{T}_{1}\right| \leq C\left(\sigma_{2}, L, \theta, n, E_{0, \sigma_{1}, \theta}, T\right)\left\|\boldsymbol{\varphi}_{n}\right\|_{L^{\frac{8}{3}}\left(0, T ; L^{2}\left(\Omega ; \mathbb{R}^{d}\right)\right)} .
$$

We move on to bound $\mathrm{T}_{2}$. Thanks to $(7.29)_{1}$ and the Cauchy-Schwarz inequality, we have that

$$
\begin{aligned}
\left|\mathrm{T}_{2}\right| & \leq C\left(\theta, n, E_{0, \sigma_{1}, \theta}, T\right)\left\|\boldsymbol{\varphi}_{n}(\cdot-\Delta t)\right\|_{L^{2}\left(\Delta t, T ; L^{2}\left(\Omega ; \mathbb{R}^{d}\right)\right)}\left\|\bar{\delta}_{t} \varrho_{n, \sigma_{3}}\right\|_{L^{2}\left(\Delta t, T ; L^{2}(\Omega)\right)} \\
& =C\left(\theta, n, E_{0, \sigma_{1}, \theta}, T\right)\left\|\boldsymbol{\varphi}_{n}\right\|_{L^{2}\left(0, T ; L^{2}\left(\Omega ; \mathbb{R}^{d}\right)\right)}\left\|\bar{\delta}_{t} \varrho_{n, \sigma_{3}}\right\|_{L^{2}\left(\Delta t, T ; L^{2}(\Omega)\right) .}
\end{aligned}
$$

In order to complete the bound on $\mathrm{T}_{2}$ we will show that $\left\|\bar{\delta}_{t} \varrho_{n, \sigma_{3}}\right\|_{L^{2}\left(\Delta t, T ; L^{2}(\Omega)\right)} \leq C\left(\sigma_{2}, \theta, n, E_{0, \sigma_{1}, \theta}, T\right)$. To this end, integrate (6.36) from 0 to $t$ for $t \in(0, T]$, consider the backward difference quotient of the resulting equality, and take its $\|\cdot\|_{L^{2}(\Omega)}$ norm. Using the triangle inequality and Minkowski's integral inequality then gives, for a.e. $t \in[\Delta t, T]$,

$$
\left\|\bar{\delta}_{t} \varrho_{n, \sigma_{3}}(\cdot, t)\right\|_{L^{2}(\Omega)} \leq \frac{1}{\Delta t} \int_{t-\Delta t}^{t}\left\|\operatorname{div}_{x}\left(\varrho_{n, \sigma_{3}} \mathbf{u}_{n, \sigma_{3}}\right)(\cdot, s)\right\|_{L^{2}(\Omega)} \mathrm{d} s+\sigma_{2} \frac{1}{\Delta t} \int_{t-\Delta t}^{t}\left\|\Delta_{x} \varrho_{n, \sigma_{3}}(\cdot, s)\right\|_{L^{2}(\Omega)} \mathrm{d} s .
$$

Thus we deduce that

$$
\begin{aligned}
& \left\|\bar{\delta}_{t} \varrho_{n, \sigma_{3}}\right\|_{L^{2}\left(\Delta t, T ; L^{2}(\Omega)\right)}=\|\| \bar{\delta}_{t} \varrho_{n, \sigma_{3}}\left\|_{L^{2}(\Omega)}\right\|_{L^{2}(\Delta t, T)} \\
& \quad \leq\left\|\frac{1}{\Delta t} \int_{-\Delta t}\right\| \operatorname{div}_{x}\left(\varrho_{n, \sigma_{3}} \mathbf{u}_{n, \sigma_{3}}\right)(\cdot, s)\left\|_{L^{2}(\Omega)} \mathrm{d} s\right\|_{L^{2}(\Delta t, T)}+\sigma_{2}\left\|\frac{1}{\Delta t} \int_{--\Delta t}\right\| \Delta_{x} \varrho_{n, \sigma_{3}}(\cdot, s)\left\|_{L^{2}(\Omega)} \mathrm{d} s\right\|_{L^{2}(\Delta t, T)} \\
& \quad \leq\left\|\operatorname{div}_{x}\left(\varrho_{n, \sigma_{3}} \mathbf{u}_{n, \sigma_{3}}\right)\right\|_{L^{2}\left(0, T ; L^{2}(\Omega)\right)}+\sigma_{2}\left\|\Delta_{x} \varrho_{n, \sigma_{3}}\right\|_{L^{2}\left(0, T ; L^{2}(\Omega)\right)} \\
& \quad \leq\left\|\varrho_{n, \sigma_{3}} \operatorname{div}_{x} \mathbf{u}_{n, \sigma_{3}}\right\|_{L^{2}\left(0, T ; L^{2}(\Omega)\right)}+\left\|\mathbf{u}_{n, \sigma_{3}} \cdot \nabla_{x} \varrho_{n, \sigma_{3}}\right\|_{L^{2}\left(0, T ; L^{2}(\Omega)\right)}+\sigma_{2}\left\|\Delta_{x} \varrho_{n, \sigma_{3}}\right\|_{L^{2}\left(0, T ; L^{2}(\Omega)\right)} \\
& \quad \leq C\left(\theta, n, E_{0, \sigma_{1}, \theta}, T\right)\left\|\varrho_{n, \sigma_{3}}\right\|_{L^{2}\left(0, T ; W^{2,2}(\Omega)\right)} \\
& \quad \leq C\left(\theta, n, E_{0, \sigma_{1}, \theta}, T\right) C\left(\sigma_{2}, \theta, n, E_{0, \sigma_{1}, \theta}, T\right)=C\left(\sigma_{2}, \theta, n, E_{0, \sigma_{1}, \theta}, T\right),
\end{aligned}
$$

where in the transition to the penultimate line we have made use of $(7.29)_{1}$, and in the transition to the last line we have used $(7.29)_{3}$. Substitution of (8.55) into (8.54) then yields

$$
\left|\mathrm{T}_{2}\right| \leq C\left(\sigma_{2}, \theta, n, E_{0, \sigma_{1}, \theta}, T\right)\left\|\boldsymbol{\varphi}_{n}\right\|_{L^{2}\left(0, T ; L^{2}\left(\Omega ; \mathbb{R}^{d}\right)\right)} .
$$

Having bounded $\mathrm{T}_{1}$ and $\mathrm{T}_{2}$ we return with (8.53) and (8.56) to (8.50) and recall that $\varphi_{n} \in C_{0}^{1}\left((0, T) ; X_{n}\right)$ with $\operatorname{supp} \varphi_{n} \subseteq\left[t_{*}, t_{* *}\right] \subset(0, T)$ and $0<\Delta t<\min \left\{t_{*}, T-t_{* *}\right\} ;$ hence,

$$
\left|\int_{0}^{T} \int_{\Omega} \varrho_{n, \sigma_{3}} \delta_{t} \mathbf{u}_{n, \sigma_{3}} \cdot \boldsymbol{\varphi}_{n} \mathrm{~d} x \mathrm{~d} t\right| \leq C\left(\sigma_{2}, L, \theta, n, E_{0, \sigma_{1}, \theta}, T\right)\left\|\boldsymbol{\varphi}_{n}\right\|_{L^{\frac{8}{3}}\left(t_{*}, t_{* *} ; L^{2}\left(\Omega ; \mathbb{R}^{d}\right)\right)} .
$$

As the set of all such functions $\varphi_{n}$ contains $C_{0}^{\infty}\left(\left(t_{*}, t_{* *}\right) ; X_{n}\right)$, which is, in turn, dense in $L^{\frac{8}{3}}\left(t_{*}, t_{* *} ; X_{n}\right)$, it follows that (8.57) holds for all $\varphi_{n} \in L^{\frac{8}{3}}\left(t_{*}, t_{* *} ; X_{n}\right)$ and all $\Delta t$ such that $0<\Delta t<\min \left\{t_{*}, T-t_{* *}\right\}$. In particular, by choosing

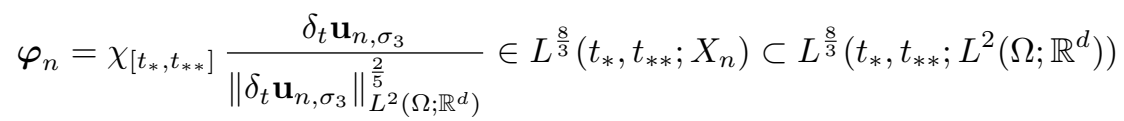

in (8.57) implies that $\left\|\delta_{t} \mathbf{u}_{n, \sigma_{3}}\right\|_{L^{\frac{8}{5}}\left(t_{*}, t_{* *} ; L^{2}\left(\Omega ; \mathbb{R}^{d}\right)\right)} \leq C\left(\sigma_{2}, L, \theta, n, E_{0, \sigma_{1}, \theta}, T\right)$ for all $\Delta t$ such that $0<\Delta t<\min \left\{t_{*}, T-t_{* *}\right\}$ and all $t_{*}<t_{* *}$ with $t_{*}, t_{* *} \in(0, T)$. Thus $t \in(0, T) \mapsto \mathbf{u}_{n, \sigma_{3}}(\cdot, t) \in X_{n}$ is weakly differentiable and (8.48) holds by a standard characterisation of Sobolev functions by uniformly bounded difference quotients (cf. Lemma 7.24 in [15]). 
8.4. Convergence to the third level of approximation. Thanks to the convergence results (8.1), (8.2), (8.49), (8.5), (8.9), (8.32), (8.33) and (8.38) $)_{3}$, letting $\sigma_{3} \rightarrow 0$ in the fourth level of approximation (6.33)-(6.37), by noting that $\mathbb{T}_{n, \sigma_{3}}^{S}=\mathbb{T}_{n, \sigma_{3}}$ and (1.4), implies that the limit $\left(\varrho_{n}, \mathbf{u}_{n}, \mathbb{T}_{n}\right)$ is a solution to the third level of approximation, (6.18)-(6.20). The attainment of the boundary conditions in (1.9), (1.10) and (6.16) for $\left(\varrho_{n}, \mathbf{u}_{n}, \mathbb{T}_{n}\right)$ follows from the attainment of the boundary conditions for $\left(\varrho_{n, \sigma_{3}}, \mathbf{u}_{n, \sigma_{3}}, \mathbb{T}_{n, \sigma_{3}}\right)$. The initial datum for $\varrho_{n}$ in $L^{q}(\Omega)$ and $\mathbb{T}_{n}$ in $L^{q}\left(\Omega ; \mathbb{R}^{d \times d}\right)$ is attained in the sense of (8.2), with the values of $q$ stated there; whereas the initial datum for $\mathbf{u}_{n}$ is attained in the sense of the $W^{2,2}\left(\Omega ; \mathbb{R}^{d}\right)$ norm by $(8.49)$.

Moreover, by the convergence results $(8.2),(8.49),(8.9),(8.38)_{1,2},(8.32),(8.12)$, weak lower-semicontinuity of the norm in $L^{2}$ spaces, (8.3), (8.18), (7.30) and Fatou's lemma, letting $\sigma_{3} \rightarrow 0$ in the inequalities (7.22), with $T_{n, \sigma_{3}}$ replaced with $T$, gives, for a.e. $t \in(0, T]$, the following inequalities:

$$
\begin{aligned}
E_{n}(t) & +4 \sigma_{2} \int_{0}^{t} \int_{\Omega}\left(\frac{a}{\gamma}\left|\nabla_{x} \varrho_{n}^{\frac{\gamma}{2}}\right|^{2}+\frac{\sigma_{1}}{\Gamma}\left|\nabla_{x} \varrho_{n}^{\frac{\Gamma}{2}}\right|^{2}\right) \mathrm{d} x \mathrm{~d} t^{\prime}+\frac{1}{4 \lambda} \int_{0}^{t} \int_{\Omega} \operatorname{tr}\left(\left(\mathbb{A}\left(\mathbb{T}_{n}\right)\right)^{2} \mathbb{T}_{n}^{-1}\right) \mathrm{d} x \mathrm{~d} t^{\prime} \\
& +\int_{0}^{t} \int_{\Omega}\left[\mu^{S}\left|\frac{\nabla_{x} \mathbf{u}_{n}+\nabla_{x}^{\mathrm{T}} \mathbf{u}_{n}}{2}-\frac{1}{d}\left(\operatorname{div}_{x} \mathbf{u}_{n}\right) \mathbb{I}\right|^{2}+\mu^{B}\left|\operatorname{div}_{x} \mathbf{u}_{n}\right|^{2}\right] \mathrm{d} x \mathrm{~d} t^{\prime} \\
& +\frac{\varepsilon}{2} \int_{0}^{t} \int_{\Omega}\left[\mathfrak{b}\left|\nabla_{x} \log \left(1-\frac{\operatorname{tr}\left(\mathbb{T}_{n}\right)}{\mathfrak{b}}\right)\right|^{2}+\frac{\mathfrak{a}}{d}\left|\nabla_{x} \operatorname{tr}\left(\log \mathbb{T}_{n}\right)\right|^{2}\right] \mathrm{d} x \mathrm{~d} t^{\prime} \\
\leq & E_{0, \sigma_{1}, \theta}+\int_{0}^{t} \int_{\Omega} \varrho_{n} \mathbf{f} \cdot \mathbf{u}_{n} \mathrm{~d} x \mathrm{~d} t^{\prime} \leq \mathrm{e}^{C t} E_{0, \sigma_{1}, \theta},
\end{aligned}
$$

where $C$ is a positive constant, independent of $t$ and of the approximation parameters $\left(\Gamma, \sigma_{1}, \sigma_{2}, \sigma_{3}, \theta, L, n\right)$. Here the positive energy $E_{n}$ is defined by

$$
E_{n}(t):=\int_{\Omega}\left[\frac{1}{2} \varrho_{n}\left|\mathbf{u}_{n}\right|^{2}+\frac{a}{\gamma-1} \varrho_{n}^{\gamma}+\frac{\sigma_{1}}{\Gamma-1} \varrho_{n}^{\Gamma}-\Pi\left(\mathbb{T}_{n}\right)+\frac{1}{2}(d \mathfrak{a} \log \mathfrak{a}+\mathfrak{b})\right] \mathrm{d} x,
$$

and the initial positive energy $E_{0, \sigma_{1}, \theta}$ is the same as in (7.20) and hence is bounded by (7.23).

\section{The SeCond LeVel of approximation}

Our objective in this section is to study the limit of the solution sequence $\left(\varrho_{n}, \mathbf{u}_{n}, \mathbb{T}_{n}\right)$ as $n \rightarrow \infty$, in order to deduce the existence of a solution to the second level of approximation, stated in Section 6.3. To this end, we need to derive bounds on $\left(\varrho_{n}, \mathbf{u}_{n}, \mathbb{T}_{n}\right)$ that are uniform in $n$.

9.1. Uniform bounds and convergence. We summarise the $n$-uniform bounds that follow from (8.58) and (8.59) by recalling (8.3), and noting a Friedrichs inequality and Korn's inequality (3.28):

$$
\begin{aligned}
&\left\|\varrho_{n}\right\|_{L^{\infty}\left(0, T ; L^{\gamma}(\Omega)\right)}+\sigma_{1}^{\frac{1}{\Gamma}}\left\|\varrho_{n}\right\|_{L^{\infty}\left(0, T ; L^{\Gamma}(\Omega)\right)} \leq C\left(E_{0, \sigma_{1}, \theta}, T\right), \\
& \sqrt{\sigma_{2}}\left\|\nabla_{x}\left(\varrho_{n}^{\frac{\gamma}{2}}\right)\right\|_{L^{2}\left((0, T) \times \Omega ; \mathbb{R}^{d}\right)}+\sqrt{\sigma_{1}} \sqrt{\sigma_{2}}\left\|\nabla_{x}\left(\varrho_{n}^{\frac{\Gamma}{2}}\right)\right\|_{L^{2}\left((0, T) \times \Omega ; \mathbb{R}^{d}\right)} \leq C\left(E_{0, \sigma_{1}, \theta}, T\right), \\
&\left\|\varrho_{n}\left|\mathbf{u}_{n}\right|^{2}\right\|_{L^{\infty}\left(0, T ; L^{1}(\Omega)\right)}+\left\|\mathbf{u}_{n}\right\|_{L^{2}\left(0, T ; W_{0}^{1,2}\left(\Omega ; \mathbb{R}^{d}\right)\right)} \leq C\left(E_{0, \sigma_{1}, \theta}, T\right), \\
&\left\|\Pi\left(\mathbb{T}_{n}\right)\right\|_{L^{\infty}\left(0, T ; L^{1}(\Omega)\right)}+\left\|\operatorname{tr}\left(\left(\mathbb{A}\left(\mathbb{T}_{n}\right)\right)^{2} \mathbb{T}_{n}^{-1}\right)\right\|_{L^{1}\left(0, T ; L^{1}(\Omega)\right)} \leq C\left(E_{0, \sigma_{1}, \theta}, T\right), \\
&\left\|\nabla_{x} \operatorname{tr}\left(\log \mathbb{T}_{n}\right)\right\|_{L^{2}\left(0, T ; L^{2}\left(\Omega ; \mathbb{R}^{d}\right)\right)}+\left\|\nabla_{x} \log \left(1-\frac{\operatorname{tr}\left(\mathbb{T}_{n}\right)}{\mathfrak{b}}\right)\right\|_{L^{2}\left(0, T ; L^{2}\left(\Omega ; \mathbb{R}^{d}\right)\right)} \leq C\left(E_{0, \sigma_{1}, \theta}, T\right) .
\end{aligned}
$$

Multiplying (6.19) by $\varrho_{n}$, recall $(8.1)_{2}$, and integrating the result over $\Omega$, performing partial integration and applying the boundary conditions (1.9), (6.16) for $\mathbf{u}_{n}$ and $\varrho_{n}$ implies that

$$
\begin{aligned}
\frac{1}{2} \frac{\mathrm{d}}{\mathrm{d} t} \int_{\Omega} \varrho_{n}^{2} \mathrm{~d} x+\sigma_{2} \int_{\Omega}\left|\nabla_{x} \varrho_{n}\right|^{2} \mathrm{~d} x & =-\int_{\Omega} \operatorname{div}_{x}\left(\varrho_{n} \mathbf{u}_{n}\right) \varrho_{n} \mathrm{~d} x=-\frac{1}{2} \int_{\Omega}\left(\operatorname{div}_{x} \mathbf{u}_{n}\right) \varrho_{n}^{2} \mathrm{~d} x \\
& \leq \frac{1}{4}\left(\int_{\Omega}\left|\operatorname{div}_{x} \mathbf{u}_{n}\right|^{2} \mathrm{~d} x+\int_{\Omega} \varrho_{n}^{4} \mathrm{~d} x\right) .
\end{aligned}
$$

Combining this with $(9.1)_{1,3}$ and recalling from Section 6.2 that $\Gamma>\max \{\gamma, 8\}$, we deduce that

$$
\sqrt{\sigma_{2}}\left\|\nabla_{x} \varrho_{n}\right\|_{L^{2}\left(0, T ; L^{2}\left(\Omega ; \mathbb{R}^{d}\right)\right)} \leq C\left(\sigma_{1}, E_{0, \sigma_{1}, \theta}, T\right) .
$$


Similarly, we have from (6.19), $(9.1)_{1,3},(9.2)$ and Sobolev embedding that, for any $\eta \in L^{2}\left(0, T ; W^{1,2}(\Omega)\right)$,

$$
\begin{aligned}
\left|\int_{0}^{T} \int_{\Omega} \partial_{t} \varrho_{n} \eta \mathrm{d} x \mathrm{~d} t\right| & \leq\left[\sigma_{2}\left\|\nabla_{x} \varrho_{n}\right\|_{L^{2}\left(0, T ; L^{2}\left(\Omega ; \mathbb{R}^{d}\right)\right)}+\left\|\mathbf{u}_{n}\right\|_{L^{2}\left(0, T ; L^{4}\left(\Omega ; \mathbb{R}^{d}\right)\right)}\left\|\varrho_{n}\right\|_{L^{\infty}\left(0, T ; L^{4}(\Omega)\right)}\right]\|\eta\|_{L^{2}\left(0, T ; W^{1,2}(\Omega)\right)} \\
& \leq C\left(\sigma_{1}, E_{0, \sigma_{1}, \theta}, T\right)\|\eta\|_{L^{2}\left(0, T ; W^{1,2}(\Omega)\right)} .
\end{aligned}
$$

Hence, we obtain from (9.4) that

$$
\left\|\partial_{t} \varrho_{n}\right\|_{L^{2}\left(0, T ; W^{-1,2}(\Omega)\right)} \leq C\left(\sigma_{1}, E_{0, \sigma_{1}, \theta}, T\right) .
$$

We deduce from a Poincaré inequality, (1.8) and $(9.1)_{5}$ that

$$
\left\|(I-f) \Pi\left(\mathbb{T}_{n}\right)\right\|_{L^{2}\left(0, T ; L^{2}(\Omega)\right)} \leq C\left\|\nabla_{x} \Pi\left(\mathbb{T}_{n}\right)\right\|_{L^{2}\left(0, T ; L^{2}\left(\Omega ; \mathbb{R}^{d}\right)\right)} \leq C\left(E_{0, \sigma_{1}, \theta}, T\right) .
$$

Similarly to (3.31), it follows from $(9.1)_{4}$ and (8.18) that

$$
\begin{aligned}
\left\|\mathbb{A}\left(\mathbb{T}_{n}\right)\right\|_{L^{2}\left(0, T ; L^{2}\left(\Omega, \mathbb{R}^{d \times d}\right)\right)}^{2} & =\int_{0}^{T} \int_{\Omega}\left|\mathbb{A}\left(\mathbb{T}_{n}\right)\left[\mathbb{T}_{n}\right]^{-\frac{1}{2}}\left[\mathbb{T}_{n}\right]^{\frac{1}{2}}\right|^{2} \mathrm{~d} x \mathrm{~d} t \\
& \leq \mathfrak{b} \int_{0}^{T} \int_{\Omega} \operatorname{tr}\left(\left(\mathbb{A}\left(\mathbb{T}_{n}\right)\right)^{2}\left[\mathbb{T}_{n}\right]^{-1}\right) \leq C\left(E_{0, \sigma_{1}, \theta}, T\right) .
\end{aligned}
$$

Similarly to (3.32), taking the inner product of $(6.20)$ with $\mathbb{T}_{n}$, recall $(8.1)_{3}$, integrating the result over $\Omega$, performing partial integration, applying the boundary conditions (1.9), (1.10) for $\mathbf{u}_{n}$ and $\mathbb{T}_{n}$, and noting (8.18), we deduce that

$$
\begin{aligned}
\frac{1}{2} \frac{\mathrm{d}}{\mathrm{d} t} \int_{\Omega}\left|\mathbb{T}_{n}\right|^{2} \mathrm{~d} x & +\varepsilon \int_{\Omega}\left|\nabla_{x} \mathbb{T}_{n}\right|^{2} \mathrm{~d} x \\
& =-\frac{1}{2} \int_{\Omega}\left(\mathbf{u}_{n} \cdot \nabla_{x}\right)\left|\mathbb{T}_{n}\right|^{2} \mathrm{~d} x+\int_{\Omega}\left(\nabla_{x} \mathbf{u}_{n} \mathbb{T}_{n}+\mathbb{T}_{n} \nabla_{x}^{\mathrm{T}} \mathbf{u}_{n}\right): \mathbb{T}_{n} \mathrm{~d} x-\frac{1}{2 \lambda} \int_{\Omega} \mathbb{A}\left(\mathbb{T}_{n}\right): \mathbb{T}_{n} \mathrm{~d} x \\
& \leq C(\mathfrak{b})\left(\left\|\nabla_{x} \mathbf{u}_{n}\right\|_{L^{1}\left(\Omega ; \mathbb{R}^{d}\right)}+\left\|\mathbb{A}\left(\mathbb{T}_{n}\right)\right\|_{L^{1}\left(\Omega ; \mathbb{R}^{d \times d}\right)}\right), \quad t \in(0, T] .
\end{aligned}
$$

Integrating (9.8) over $t \in(0, T)$ and noting $(9.1)_{3},(9.7),(8.18)$ and the initial condition (6.3) satisfying (6.6), we have the following $n$-uniform bounds on $\mathbb{T}_{n}$ :

$$
\left\|\mathbb{T}_{n}\right\|_{L^{\infty}\left(0, T ; L^{\infty}\left(\Omega ; \mathbb{R}^{d \times d}\right)\right)}+\left\|\mathbb{T}_{n}\right\|_{L^{2}\left(0, T ; W^{1,2}\left(\Omega ; \mathbb{R}^{d \times d}\right)\right)} \leq C\left(E_{0, \sigma_{1}, \theta}, T\right) .
$$

It follows from (6.20), the boundary conditions (1.9), (1.10) for $\mathbf{u}_{n}$ and $\mathbb{T}_{n},(9.9),(9.1)_{3}$ and (9.7) for any $\mathbb{Y} \in$ $L^{2}\left(0, T ; W^{1,2}\left(\Omega ; \mathbb{R}^{d \times d}\right)\right)$ that

$$
\begin{aligned}
& \left|\int_{0}^{T} \int_{\Omega} \partial_{t} \mathbb{T}_{n}: \mathbb{Y} \mathrm{d} x \mathrm{~d} t\right| \\
& \leq C\left(E_{0, \sigma_{1}, \theta}, T\right)\left(1+\left\|\mathbf{u}_{n}\right\|_{L^{2}\left(0, T ; W_{0}^{1,2}\left(\Omega ; \mathbb{R}^{d}\right)\right)}+\left\|\mathbb{A}\left(\mathbb{T}_{n}\right)\right\|_{L^{2}\left(0, T ; L^{2}\left(\Omega ; \mathbb{R}^{d \times d}\right)\right)}\right)\|\mathbb{Y}\|_{L^{2}\left(0, T ; W^{1,2}\left(\Omega ; \mathbb{R}^{d \times d}\right)\right)} \\
& \quad \leq C\left(E_{0, \sigma_{1}, \theta}, T\right)\|\mathbb{Y}\|_{L^{2}\left(0, T ; W^{1,2}\left(\Omega ; \mathbb{R}^{d \times d}\right)\right)}
\end{aligned}
$$

Hence, we obtain from (9.10) that

$$
\left\|\partial_{t} \mathbb{T}_{n}\right\|_{L^{2}\left(0, T ; W^{-1,2}\left(\Omega ; \mathbb{R}^{d \times d}\right)\right)} \leq C\left(E_{0, \sigma_{1}, \theta}, T\right) .
$$

Therefore, $(9.1)_{1,3},(9.3),(9.5),(9.9)$ and (9.11) imply the following weak convergence results, as $n \rightarrow \infty$ :

$$
\begin{aligned}
\mathbf{u}_{n} \rightarrow \mathbf{u}_{\sigma_{2}} & \text { weakly in } L^{2}\left(0, T ; W_{0}^{1,2}\left(\Omega ; \mathbb{R}^{d}\right)\right), \\
\varrho_{n} \rightarrow \varrho_{\sigma_{2}} & \text { weakly-* in } L^{\infty}\left(0, T ; L^{\Gamma}(\Omega)\right) \cap L^{2}\left(0, T ; W^{1,2}(\Omega)\right) \cap W^{1,2}\left(0, T ; W^{-1,2}(\Omega)\right), \\
\mathbb{T}_{n} \rightarrow \mathbb{T}_{\sigma_{2}} & \text { weakly-* in } L^{\infty}\left(0, T ; L^{\infty}\left(\Omega ; \mathbb{R}^{d \times d}\right)\right) \cap L^{2}\left(0, T ; W^{1,2}\left(\Omega ; \mathbb{R}^{d \times d}\right)\right) \cap W^{1,2}\left(0, T ; W^{-1,2}\left(\Omega ; \mathbb{R}^{d \times d}\right)\right) .
\end{aligned}
$$

The time derivative bounds (9.5) and (9.11) enable us to use the Aubin-Lions-Simon compactness result, Lemma 5.3, to obtain the following strong convergence results, as $n \rightarrow \infty$ :

$$
\begin{aligned}
\varrho_{n} \rightarrow \varrho_{\sigma_{2}} & \text { strongly in } L^{2}\left(0, T ; L^{q}(\Omega)\right), \\
\mathbb{T}_{n} \rightarrow \mathbb{T}_{\sigma_{2}} & \text { strongly in } L^{2}\left(0, T ; L^{q}\left(\Omega ; \mathbb{R}^{d \times d}\right)\right),
\end{aligned}
$$

where $q \in[1, \infty)$ if $d=2$ and $q \in[1,6)$ if $d=3$. It follows from (8.3) and (9.13) $)_{1}$ that

$$
0 \leq \varrho_{\sigma_{2}} \text { a.e. in }(0, T] \times \Omega .
$$


In addition, it follows from $(9.1)_{1,3}$ and Sobolev embedding that

$$
\begin{aligned}
& \left\|\varrho_{n} \mathbf{u}_{n}\right\|_{L^{\infty}\left(0, T ; L^{\Gamma+1}\left(\Omega ; \mathbb{R}^{d}\right)\right)} \leq\left\|\varrho_{n}\right\|_{L^{\infty}\left(0, T ; L^{\Gamma}(\Omega)\right)}^{\frac{1}{2}}\left\|\varrho_{n}\left|\mathbf{u}_{n}\right|^{2}\right\|_{L^{\infty}\left(0, T ; L^{1}(\Omega)\right)}^{\frac{1}{2}} \leq C\left(\sigma_{1}, E_{0, \sigma_{1}, \theta}, T\right), \\
& \left\|\varrho_{n} \mathbf{u}_{n}\right\|_{L^{2}\left(0, T ; L^{\Gamma+6}\left(\Omega ; \mathbb{R}^{d}\right)\right)} \leq\left\|\varrho_{n}\right\|_{L^{\infty}\left(0, T ; L^{\Gamma}(\Omega)\right)}\left\|\mathbf{u}_{n}\right\|_{L^{2}\left(0, T ; L^{6}\left(\Omega ; \mathbb{R}^{d}\right)\right)} \leq C\left(\sigma_{1}, E_{0, \sigma_{1}, \theta}, T\right), \\
& \left\|\varrho_{n}\left|\mathbf{u}_{n}\right|^{2}\right\|_{L^{2}\left(0, T ; L^{\frac{6 \Gamma}{4 \Gamma+3}}\left(\Omega ; \mathbb{R}^{d \times d}\right)\right)} \leq\left\|\varrho_{n} \mathbf{u}_{n}\right\|_{L^{\infty}\left(0, T ; L^{\Gamma+1}\right.}{ }_{\frac{2 \Gamma}{\left.\left.\Gamma+\mathbb{R}^{d}\right)\right)}}\left\|\mathbf{u}_{n}\right\|_{L^{2}\left(0, T ; L^{6}\left(\Omega ; \mathbb{R}^{d}\right)\right)} \leq C\left(\sigma_{1}, E_{0, \sigma_{1}, \theta}, T\right) .
\end{aligned}
$$

Following the same argument as in (8.6)-(8.9), we obtain at first, using $(9.1)_{1,2}$, that

$$
\left\|\varrho_{n}^{\Gamma}\right\|_{L^{\frac{4}{3}}\left(0, T ; L^{2}(\Omega)\right)} \leq C\left(\sigma_{1}, \sigma_{2}, E_{0, \sigma_{1}, \theta}, T\right)
$$

and hence, by noting (9.13), we deduce that, as $n \rightarrow \infty$,

$$
\begin{array}{rlr}
\varrho_{n} & \rightarrow \varrho_{\sigma_{2}} & \text { strongly in } L^{\beta}\left(0, T ; L^{\beta}(\Omega)\right), \\
\varrho_{n}^{\gamma} \rightarrow \varrho_{\sigma_{2}}^{\gamma}, & \varrho_{n}^{\Gamma} \rightarrow \varrho_{\sigma_{2}}^{\Gamma} & \text { strongly in } L^{1}\left(0, T ; L^{1}(\Omega)\right),
\end{array}
$$

where $\beta \in\left[1, \frac{4 \Gamma}{3}\right)$. Thus we deduce from $(9.15)_{1,2},(9.17)_{1}$ and $(9.12)_{1}$ that, as $n \rightarrow \infty$,

$$
\varrho_{n} \mathbf{u}_{n} \rightarrow \varrho_{\sigma_{2}} \mathbf{u}_{\sigma_{2}} \quad \text { weakly-* in } L^{\infty}\left(0, T ; L^{\frac{2 \Gamma}{\Gamma+1}}\left(\Omega ; \mathbb{R}^{d}\right)\right) \cap L^{2}\left(0, T ; L^{\frac{6 \Gamma}{\Gamma+6}}\left(\Omega ; \mathbb{R}^{d}\right)\right) .
$$

It follows from $(9.15)_{1,2}$, as $\Gamma>8>3 \Rightarrow \frac{6 \Gamma}{\Gamma+6}>2$, and function space interpolation that

$$
\left\|\varrho_{n} \mathbf{u}_{n}\right\|_{L^{r_{\star}}\left(0, T ; L^{r_{\star}}\left(\Omega ; \mathbb{R}^{d}\right)\right)} \leq C\left(\sigma_{1}, E_{0, \sigma_{1}, \theta}, T\right)
$$

for some $r_{\star}>2$. Applying Lemma 5.7 to (6.19), by noting (9.19), yields that



for this $r_{\star}>2$. Hence, it follows from (9.20) and (9.1) 3 that

$$
\left\|\nabla_{x} \mathbf{u}_{n} \nabla_{x} \varrho_{n}\right\|_{L^{s_{\star}}\left(0, T ; L^{s_{\star}}\left(\Omega ; \mathbb{R}^{d}\right)\right)} \leq C\left(\sigma_{1}, E_{0, \sigma_{1}, \theta}, T\right)
$$

for some $s_{\star}>1$.

From (6.18), by the same argument as in Section 7.8.2 in [22], it follows from $(9.1)_{3},(9.16),(9.15)_{3},(9.21),(9.6)$ and (9.7) that

$$
\left\|\partial_{t} P_{n}\left(\varrho_{n} \mathbf{u}_{n}\right)\right\|_{L^{r}\left(0, T ; \widetilde{W}^{-2,2}\left(\Omega ; \mathbb{R}^{d}\right)\right)} \leq C\left(\sigma_{1}, \sigma_{2}, E_{0, \sigma_{1}, \theta}, T\right), \quad \text { for some } r>1
$$

where $P_{n}$ is the orthogonal projection from $L^{2}\left(\Omega ; \mathbb{R}^{d}\right)$ onto $X_{n}$ and $\widetilde{W}^{-2,2}\left(\Omega ; \mathbb{R}^{d}\right)$ is the dual space of $W_{0}^{1,2}\left(\Omega ; \mathbb{R}^{d}\right) \cap$ $W^{2,2}\left(\Omega ; \mathbb{R}^{d}\right)$. On the other hand, since $\Gamma>\max \{\gamma, 8\}$, we deduce from $(9.15)_{2}$ that

$$
\left\|P_{n}\left(\varrho_{n} \mathbf{u}_{n}\right)\right\|_{L^{2}\left(0, T ; L^{2}\left(\Omega ; \mathbb{R}^{d}\right)\right)} \leq\left\|\varrho_{n} \mathbf{u}_{n}\right\|_{L^{2}\left(0, T ; L^{2}\left(\Omega ; \mathbb{R}^{d}\right)\right)} \leq C\left(\sigma_{1}, E_{0, \sigma_{1}, \theta}, T\right) .
$$

Thus, Lemma 5.3, the compact embeddings $L^{2}\left(\Omega ; \mathbb{R}^{d}\right) \hookrightarrow \hookrightarrow W^{-1,2}\left(\Omega ; \mathbb{R}^{d}\right) \hookrightarrow \hookrightarrow \widetilde{W}^{-2,2}\left(\Omega ; \mathbb{R}^{d}\right)$ that follow from the compact embeddings $W^{2,2}\left(\Omega ; \mathbb{R}^{d}\right) \cap W_{0}^{1,2}\left(\Omega ; \mathbb{R}^{d}\right) \hookrightarrow \hookrightarrow W^{1,2}\left(\Omega ; \mathbb{R}^{d}\right) \hookrightarrow \hookrightarrow L^{2}\left(\Omega ; \mathbb{R}^{d}\right)$, and (9.18) yield that

$$
P_{n}\left(\varrho_{n} \mathbf{u}_{n}\right) \rightarrow \varrho_{\sigma_{2}} \mathbf{u}_{\sigma_{2}} \quad \text { strongly in } L^{2}\left(0, T ; W^{-1,2}\left(\Omega ; \mathbb{R}^{d}\right)\right) .
$$

By writing $\varrho_{n} \mathbf{u}_{n}=P_{n}\left(\varrho_{n} \mathbf{u}_{n}\right)+\left(1-P_{n}\right)\left(\varrho_{n} \mathbf{u}_{n}\right)$ one can deduce that

$$
\varrho_{n} \mathbf{u}_{n} \rightarrow \varrho_{\sigma_{2}} \mathbf{u}_{\sigma_{2}} \quad \text { strongly in } L^{2}\left(0, T ; W^{-1,2}\left(\Omega ; \mathbb{R}^{d}\right)\right),
$$

see Section 7.8.2 in [22] for the details. Now, we observe that (9.12) $)_{1}$ implies that $\int_{0}^{T} \int_{\Omega} \mathbf{u}_{n} \eta \mathrm{d} x \mathrm{~d} t \rightarrow \int_{0}^{T} \int_{\Omega} \mathbf{u}_{\sigma_{2}} \eta \mathrm{d} x \mathrm{~d} t$ for any $\eta \in C^{\infty}\left(0, T ; C_{0}^{\infty}(\Omega)\right)$. Combining this with (9.22) yields that $\int_{0}^{T} \int_{\Omega} \varrho_{n} \mathbf{u}_{n} \otimes \mathbf{u}_{n} \eta \mathrm{d} x \mathrm{~d} t \rightarrow \int_{0}^{T} \int_{\Omega} \varrho_{\sigma_{2}} \mathbf{u}_{\sigma_{2}} \otimes$ $\mathbf{u}_{\sigma_{2}} \eta \mathrm{d} x \mathrm{~d} t$ for any $\eta \in C^{\infty}\left(0, T ; C_{0}^{\infty}(\Omega)\right)$. On the other hand $(9.15)_{3}$ implies that $\varrho_{n} \mathbf{u}_{n} \otimes \mathbf{u}_{n}$ has a weak limit in $L^{2}\left(0, T ; L^{\frac{6 \Gamma}{4 \Gamma+3}}\left(\Omega ; \mathbb{R}^{d \times d}\right)\right)$. We deduce from the above that this limit is $\varrho_{\sigma_{2}} \mathbf{u}_{\sigma_{2}} \otimes \mathbf{u}_{\sigma_{2}}$. Thus, we conclude the following convergence result for the convective term:

$$
\varrho_{n} \mathbf{u}_{n} \otimes \mathbf{u}_{n} \rightarrow \varrho_{\sigma_{2}} \mathbf{u}_{\sigma_{2}} \otimes \mathbf{u}_{\sigma_{2}} \quad \text { weakly in } L^{2}\left(0, T ; L^{\frac{6 \Gamma}{4 \Gamma+3}}\left(\Omega ; \mathbb{R}^{d \times d}\right)\right) .
$$

Finally, we shall study the limit of the term $\sigma_{2} \nabla_{x} \mathbf{u}_{n} \nabla_{x} \varrho_{n}$, as $n \rightarrow \infty$, on the right-hand side of (6.18). We deduce from (9.2) that, for every $t \in(0, T]$,

$$
\left\|\varrho_{n}(t)\right\|_{L^{2}(\Omega)}^{2}+2 \sigma_{2}\left\|\nabla_{x} \varrho_{n}\right\|_{L^{2}\left(0, t ; L^{2}\left(\Omega ; \mathbb{R}^{d}\right)\right)}^{2}=\left\|\varrho_{0, \theta}\right\|_{L^{2}(\Omega)}^{2}-\int_{0}^{t} \int_{\Omega}\left(\operatorname{div}_{x} \mathbf{u}_{n}\right) \varrho_{n}^{2} \mathrm{~d} x \mathrm{dt}^{\prime} .
$$

Integrating (9.24) over $t \in(0, T)$, and performing integration by parts in time, we deduce that

$$
\left\|\varrho_{n}\right\|_{L^{2}(0, T ; \Omega)}^{2}+2 \sigma_{2} \int_{0}^{T}(T-t)\left\|\nabla_{x} \varrho_{n}(t)\right\|_{\left.L^{2}\left(\Omega ; \mathbb{R}^{d}\right)\right)}^{2} \mathrm{~d} t=T\left\|\varrho_{0, \theta}\right\|_{L^{2}(\Omega)}^{2}-\int_{0}^{T}(T-t) \int_{\Omega}\left(\operatorname{div}_{x} \mathbf{u}_{n}\right) \varrho_{n}^{2} \mathrm{~d} x \mathrm{~d} t .
$$


It follows from $(9.12)_{2}$ and (9.18) that $\varrho_{\sigma_{2}}$ and $\mathbf{u}_{\sigma_{2}}$ satisfy (6.13) and the boundary conditions (1.9) and (6.16) in the sense that

$$
\int_{0}^{T}\left\langle\partial_{t} \varrho_{\sigma_{2}}, \eta\right\rangle_{W^{1,2}(\Omega)} \mathrm{d} t+\int_{0}^{T} \int_{\Omega}\left(\sigma_{2} \nabla_{x} \varrho_{\sigma_{2}}-\varrho_{\sigma_{2}} \mathbf{u}_{\sigma_{2}}\right) \cdot \nabla_{x} \eta \mathrm{d} x \mathrm{~d} t=0 \quad \forall \eta \in L^{2}\left(0, T ; W^{1,2}(\Omega)\right) .
$$

The initial datum $\varrho_{0, \theta},(6.1)_{1}$, is attained in the sense of $C_{w}\left([0, T] ; L^{\Gamma}(\Omega)\right)$ by noting $(9.12)_{2}$ and Lemmas 5.4 and 5.5. Choosing $\eta=\chi_{[0, t]} \varrho_{\sigma_{2}}$ in (9.26), where $\chi_{S}$ denotes the characteristic function of $S$, yields the analogue of (9.24) with $\varrho_{n}$ and $\mathbf{u}_{n}$ replaced with $\varrho_{\sigma_{2}}$ and $\mathbf{u}_{\sigma_{2}}$, respectively. Integrating this over $t \in(0, T)$ then yields the analogue of $(9.25)$ :

$$
\left\|\varrho_{\sigma_{2}}\right\|_{L^{2}(0, T ; \Omega)}^{2}+2 \sigma_{2} \int_{0}^{T}(T-t)\left\|\nabla_{x} \varrho_{\sigma_{2}}(t)\right\|_{\left.L^{2}\left(\Omega ; \mathbb{R}^{d}\right)\right)}^{2} \mathrm{~d} t=T\left\|\varrho_{0, \theta}\right\|_{L^{2}(\Omega)}^{2}-\int_{0}^{T}(T-t) \int_{\Omega}\left(\operatorname{div}_{x} \mathbf{u}_{\sigma_{2}}\right) \varrho_{\sigma_{2}}^{2} \mathrm{~d} x \mathrm{~d} t .
$$

Letting $n \rightarrow \infty$ in (9.25), it follows from $(9.17)_{1},(9.12)_{1}$ and $(9.27)$, as $\Gamma>\max \{\gamma, 8\}$, that

$$
\lim _{n \rightarrow \infty} \int_{0}^{T}(T-t)\left\|\nabla_{x} \varrho_{n}\right\|_{L^{2}\left(\Omega ; \mathbb{R}^{d}\right)}^{2} \mathrm{~d} t=\int_{0}^{T}(T-t)\left\|\nabla_{x} \varrho_{\sigma_{2}}\right\|_{L^{2}\left(\Omega ; \mathbb{R}^{d}\right)}^{2} \mathrm{~d} t .
$$

By applying the elementary identity $|\mathbf{q}-\mathbf{r}|^{2}=|\mathbf{q}|^{2}-|\mathbf{r}|^{2}-2(\mathbf{q}-\mathbf{r}) \cdot \mathbf{r}$ with $\mathbf{q}=\nabla_{x} \varrho_{n}$ and $\mathbf{r}=\nabla_{x} \varrho_{\sigma_{2}}$, it follows from $(9.28)$ and $(9.12)_{2}$ that

$$
\lim _{n \rightarrow \infty} \int_{0}^{T}(T-t)\left\|\nabla_{x} \varrho_{\sigma_{2}}-\nabla_{x} \varrho_{n}\right\|_{L^{2}\left(\Omega ; \mathbb{R}^{d}\right)}^{2} \mathrm{~d} t=0 .
$$

We deduce from (9.29) that for a subsequence $\nabla_{x} \varrho_{n} \rightarrow \nabla_{x} \varrho_{\sigma_{2}}$ a.e. in $(0, T] \times \Omega$. Hence, (9.20) and the Vitali convergence theorem yield that

$$
\nabla_{x} \varrho_{n} \rightarrow \nabla_{x} \varrho_{\sigma_{2}} \quad \text { strongly in } L^{2}\left(0, T ; L^{2}\left(\Omega ; \mathbb{R}^{d}\right)\right) .
$$

Therefore, by noting (9.21), (9.30) and $(9.12)_{1}$, we have that

$$
\nabla_{x} \mathbf{u}_{n} \nabla_{x} \varrho_{n} \rightarrow \nabla_{x} \mathbf{u}_{\sigma_{2}} \nabla_{x} \varrho_{\sigma_{2}} \quad \text { weakly in } L^{s_{\star}}\left(0, T ; L^{s_{\star}}\left(\Omega ; \mathbb{R}^{d}\right)\right),
$$

where $s_{\star}>1$.

We shall combine the convergence results established in this section to show that the limit $\left(\varrho_{\sigma_{2}}, \mathbf{u}_{\sigma_{2}}, \mathbb{T}_{\sigma_{2}}\right)$ solves the second level of approximation; this will be done in Section 9.3. Before doing so however we need to prove the positive definiteness of the limiting symmetric conformation tensor $\mathbb{T}_{\sigma_{2}}$ and that $\operatorname{tr}\left(\mathbb{T}_{\sigma_{2}}\right)<\mathfrak{b}$.

9.2. Bounds on the conformation tensor $\mathbb{T}_{\sigma_{2}}$. As $\mathbb{T}_{n}$ is symmetric positive definite and $\operatorname{tr}\left(\mathbb{T}_{n}\right)<\mathfrak{b}$ a.e. in $(0, T] \times \Omega$, recall (8.18), we have from $(9.13)_{2}$ that $\mathbb{T}_{\sigma_{2}}$ is symmetric nonnegative definite and $\operatorname{tr}\left(\mathbb{T}_{\sigma_{2}}\right) \leq \mathfrak{b}$ a.e. in $(0, T] \times \Omega$. We now adapt Lemma 8.2 to show that $\mathbb{T}_{\sigma_{2}}$ is in fact symmetric positive definite and $\operatorname{tr}\left(\mathbb{T}_{\sigma_{2}}\right)<\mathfrak{b}$ a.e. on $(0, T] \times \Omega$.

Lemma 9.1. We have that

$$
\operatorname{tr}\left(\mathbb{T}_{\sigma_{2}}\right)<\mathfrak{b} \quad \text { and } \quad \mathbb{T}_{\sigma_{2}}>0 \quad \text { a.e. in }(0, T] \times \Omega .
$$

In addition, we have the following convergence result for $n \rightarrow \infty$ :

$$
\mathbb{A}\left(\mathbb{T}_{n}\right) \rightarrow \mathbb{A}\left(\mathbb{T}_{\sigma_{2}}\right) \quad \text { strongly in } L^{r}\left(0, T ; L^{r}\left(\Omega ; \mathbb{R}^{d \times d}\right)\right),
$$

for any $r \in[1,2)$.

Proof. We know already that $\operatorname{tr}\left(\mathbb{T}_{\sigma_{2}}\right) \leq \mathfrak{b}$ a.e. in $(0, T] \times \Omega$. Assume that $\operatorname{tr}\left(\mathbb{T}_{\sigma_{2}}\right)=\mathfrak{b}$ a.e. in $D_{\sigma_{2}}^{b} \subset(0, T] \times \Omega$. We then have that

$$
\mathfrak{b}\left|D_{\sigma_{2}}^{b}\right|=\int_{D_{\sigma_{2}}^{b}} \operatorname{tr}\left(\mathbb{T}_{\sigma_{2}}\right) \mathrm{d} x \mathrm{~d} t=\int_{D_{\sigma_{2}}^{b}} \operatorname{tr}\left(\mathbb{T}_{\sigma_{2}}-\mathbb{T}_{n}\right) \mathrm{d} x \mathrm{~d} t+\int_{D_{\sigma_{2}}^{b}} \operatorname{tr}\left(\mathbb{T}_{n}\right) \mathrm{d} x \mathrm{~d} t=: S_{1}+S_{2} .
$$

It follows from $(9.13)_{2}$ that $S_{1} \rightarrow 0$ as $n \rightarrow \infty$. A simple rearrangement, by recalling (1.7), yields that

$$
S_{2}=\int_{D_{\sigma_{2}}^{b}}\left[\operatorname{tr}\left(\mathbb{A}\left(\mathbb{T}_{n}\right)\right)+d \mathfrak{a}\right]\left(1-\frac{\operatorname{tr}\left(\mathbb{T}_{n}\right)}{\mathfrak{b}}\right) \mathrm{d} x \mathrm{~d} t .
$$

It then follows from (9.35), (8.22) and (9.7) that

$$
S_{2}^{2} \leq C\left(E_{0, \sigma_{1}, \theta}, T\right) \int_{D_{\sigma_{2}}^{b}}\left[1-\frac{\operatorname{tr}\left(\mathbb{T}_{n}\right)}{\mathfrak{b}}\right]^{2} \mathrm{~d} x \mathrm{~d} t=C\left(E_{0, \sigma_{1}, \theta}, T\right) \int_{D_{\sigma_{2}}^{b}}\left[\frac{\operatorname{tr}\left(\mathbb{T}_{\sigma_{2}}\right)}{\mathfrak{b}}-\frac{\operatorname{tr}\left(\mathbb{T}_{n}\right)}{\mathfrak{b}}\right]^{2} \mathrm{~d} x \mathrm{~d} t
$$

as $\operatorname{tr}\left(\mathbb{T}_{\sigma_{2}}\right)=\mathfrak{b}$ a.e. in $D_{\sigma_{2}}^{b}$. It follows from $(9.13)_{2}$ that $S_{2} \rightarrow 0$ as $n \rightarrow \infty$. Hence, we deduce from (9.34) that $\left|D_{\sigma_{2}}^{b}\right|=0$, and so the first desired result in (9.32) holds. 
We now establish the second result in (9.32). We already know that $\mathbb{T}_{\sigma_{2}} \geq 0$ a.e. in $(0, T] \times \Omega$. If the symmetric matrix function $\mathbb{T}_{\sigma_{2}}$ were not positive definite a.e. in $D_{\sigma_{2}}^{0} \subset(0, T] \times \Omega$, then there would exist a $\mathbf{q} \in L^{\infty}\left((0, T] \times \Omega ; \mathbb{R}^{d}\right)$ such that

$$
\mathbb{T}_{\sigma_{2}} \mathbf{q}=\mathbf{0} \text { a.e. in }(0, T] \times \Omega \quad \text { with } \quad|\mathbf{q}|=1 \text { a.e. in } D_{\sigma_{2}}^{0} \text { and } \mathbf{q}=\mathbf{0} \text { a.e. in }((0, T] \times \Omega) \backslash D_{\sigma_{2}}^{0} .
$$

Hence, we would have that

$$
\mathfrak{a}\left|D_{\sigma_{2}}^{0}\right|=\mathfrak{a} \int_{0}^{T} \int_{\Omega}|\mathbf{q}| \mathrm{d} x \mathrm{~d} t \leq \int_{0}^{T} \int_{\Omega}\left|\mathbb{A}\left(\mathbb{T}_{n}\right) \mathbf{q}\right| \mathrm{d} x \mathrm{~d} t+\int_{0}^{T} \int_{\Omega}\left|\left(\mathbb{A}\left(\mathbb{T}_{n}\right)+\mathfrak{a} \mathbb{I}\right) \mathbf{q}\right| \mathrm{d} x \mathrm{~d} t:=S_{3}+S_{4} .
$$

As $\mathbb{T}_{n}$ is symmetric positive definite, we have from (8.22) that

$$
\left|\mathbb{A}\left(\mathbb{T}_{n}\right) \mathbf{q}\right| \leq\left|\mathbb{A}\left(\mathbb{T}_{n}\right)\left[\mathbb{T}_{n}\right]^{-\frac{1}{2}}\right|\left|\left[\mathbb{T}_{n}\right]^{\frac{1}{2}} \mathbf{q}\right|=\left[\operatorname{tr}\left(\left(\mathbb{A}\left(\mathbb{T}_{n}\right)\right)^{2}\left[\mathbb{T}_{n}\right]^{-1}\right)\left(\mathbb{T}_{n}:\left(\mathbf{q} \mathbf{q}^{\mathrm{T}}\right)\right)\right]^{\frac{1}{2}}
$$

Combining (9.38) and (9.39), and noting $(9.1)_{4}$, yields that

$$
S_{3} \leq C\left(E_{0, \sigma_{1}, \theta}, T\right)\left(\int_{0}^{T} \int_{\Omega} \mathbb{T}_{n}:\left(\mathbf{q q}^{\mathrm{T}}\right)\right)^{\frac{1}{2}} .
$$

It follows from $(9.13)_{2}$ and (9.37) that $S_{3} \rightarrow 0$ as $n \rightarrow \infty$. Similarly to (9.39), by recalling (1.7) and (8.22), we have that

$$
S_{4} \leq d^{\frac{1}{4}}\left\|\mathbb{A}\left(\mathbb{T}_{n}\right)+\mathfrak{a} \mathbb{I}\right\|_{L^{1}\left(0, T ; L^{1}\left(\Omega ; \mathbb{R}^{d \times d}\right)\right)}^{\frac{1}{2}}\left(\int_{0}^{T} \int_{\Omega}\left[1-\frac{\operatorname{tr}\left(\mathbb{T}_{n}\right)}{\mathfrak{b}}\right]^{-1} \mathbb{T}_{n}:\left(\mathbf{q q}^{\mathrm{T}}\right) \mathrm{d} x \mathrm{~d} t\right)^{\frac{1}{2}} .
$$

We will now show that

$$
\mathbb{A}\left(\mathbb{T}_{n}\right)+\mathfrak{a} \mathbb{I}=\left(1-\frac{\operatorname{tr}\left(\mathbb{T}_{n}\right)}{\mathfrak{b}}\right)^{-1} \mathbb{T}_{n} \rightarrow\left(1-\frac{\operatorname{tr}\left(\mathbb{T}_{\sigma_{2}}\right)}{\mathfrak{b}}\right)^{-1} \mathbb{T}_{\sigma_{2}} \quad \text { strongly in } L^{r}\left(0, T ; L^{r}\left(\Omega ; \mathbb{R}^{d \times d}\right)\right),
$$

for any $r \in[1,2)$ as $n \rightarrow \infty$, by possibly extracting a subsequence. It immediately follows from $(9.13)_{2}$ that $\mathbb{A}\left(\mathbb{T}_{n}\right)+\mathfrak{a} \mathbb{I} \rightarrow$ $\left(1-\frac{\operatorname{tr}\left(\mathbb{T}_{\sigma_{2}}\right)}{\mathfrak{b}}\right)^{-1} \mathbb{T}_{\sigma_{2}}$ a.e. in $(0, T] \times \Omega$, as $n \rightarrow \infty$, since we have already established the first result in (9.32). Hence, the desired result (9.33) holds by noting (9.7), (1.7) and the Vitali convergence theorem. Finally, it follows from (9.41), (9.7), (9.42) and (9.37) that $S_{4} \rightarrow 0$ as $n \rightarrow \infty$. Therefore, (9.38) yields the second result in (9.32).

Finally, we prove convergence of the polymeric pressure term $\Pi\left(\mathbb{T}_{n}\right)$ as $n \rightarrow \infty$.

Lemma 9.2. We have the following convergence results as $n \rightarrow \infty$ :

$$
\begin{aligned}
\nabla_{x} \operatorname{tr}\left(\log \mathbb{T}_{n}\right) & \rightarrow \nabla_{x} \operatorname{tr}\left(\log \mathbb{T}_{\sigma_{2}}\right) & & \text { weakly in } L^{2}\left(0, T ; L^{2}(\Omega)\right), \\
\nabla_{x} \log \left(1-\frac{\operatorname{tr}\left(\mathbb{T}_{n}\right)}{\mathfrak{b}}\right) & \rightarrow \nabla_{x} \log \left(1-\frac{\operatorname{tr}\left(\mathbb{T}_{\sigma_{2}}\right)}{\mathfrak{b}}\right) & & \text { weakly in } L^{2}\left(0, T ; L^{2}(\Omega)\right), \\
(I-f) \Pi\left(\mathbb{T}_{n}\right) & \rightarrow(I-f) \Pi\left(\mathbb{T}_{\sigma_{2}}\right) & & \text { strongly in } L^{r}\left(0, T ; L^{r}(\Omega)\right),
\end{aligned}
$$

for any $r \in[1,2)$.

Proof. The desired results (9.43) follow from $(9.1)_{5},(9.6),(9.13)_{2},(9.32),(1.8)$ and the Vitali convergence theorem.

9.3. Convergence to the second level of approximation. We have already established that $\varrho_{\sigma_{2}}$ and $\mathbf{u}_{\sigma_{2}}$ satisfy (6.13), recall (9.26). Next, we integrate (6.18) in time over $[0, t]$, for $t \in(0, T]$, with $\varphi_{n}=P_{n} \varphi$, where $\varphi \in C^{\infty}\left([0, T] ; C_{0}^{\infty}\left(\Omega ; \mathbb{R}^{d}\right)\right)$, and perform integration by parts in time. It is then straightforward to show by letting $n \rightarrow \infty$ in this, by noting the convergence results $(9.12)_{1},(9.17),(9.18),(9.23),(9.31),(9.33)$ and $(9.43)_{3}$, that for any $\varphi \in C^{\infty}\left([0, T] ; C_{0}^{\infty}\left(\Omega ; \mathbb{R}^{d}\right)\right)$, we have for any $t \in(0, T]$ the equality

$$
\begin{gathered}
\int_{0}^{t} \int_{\Omega}\left[\varrho_{\sigma_{2}} \mathbf{u}_{\sigma_{2}} \cdot \partial_{t} \boldsymbol{\varphi}+\left(\varrho_{\sigma_{2}} \mathbf{u}_{\sigma_{2}} \otimes \mathbf{u}_{\sigma_{2}}\right): \nabla_{x} \boldsymbol{\varphi}+\left(p\left(\varrho_{\sigma_{2}}\right)+\sigma_{1} \varrho_{\sigma_{2}}^{\Gamma}\right) \operatorname{div} \boldsymbol{x}_{x} \boldsymbol{\varphi} \mathrm{d} x \mathrm{~d} t^{\prime}\right. \\
-\int_{0}^{t} \int_{\Omega}\left[\mathbb{S}\left(\nabla_{x} \mathbf{u}_{\sigma_{2}}\right): \nabla_{x} \boldsymbol{\varphi}+\sigma_{2} \nabla_{x} \mathbf{u}_{\sigma_{2}} \nabla_{x} \varrho_{\sigma_{2}} \cdot \boldsymbol{\varphi}\right] \mathrm{d} x \mathrm{~d} t^{\prime} \\
=\int_{0}^{t} \int_{\Omega} \mathbb{A}\left(\mathbb{T}_{\sigma_{2}}\right): \nabla_{x} \boldsymbol{\varphi}-\Pi\left(\mathbb{T}_{\sigma_{2}}\right) \operatorname{div}{ }_{x} \boldsymbol{\varphi}-\varrho_{\sigma_{2}} \mathbf{f} \cdot \boldsymbol{\varphi} \mathrm{d} x \mathrm{~d} t^{\prime} \\
\quad+\int_{\Omega} \varrho_{\sigma_{2}} \mathbf{u}_{\sigma_{2}}(t, \cdot) \cdot \boldsymbol{\varphi}(t, \cdot) \mathrm{d} x-\int_{\Omega} \varrho_{0, \theta} \mathbf{u}_{0, \theta} \cdot \boldsymbol{\varphi}(0, \cdot) \mathrm{d} x
\end{gathered}
$$


Again by the convergence results $(9.12)_{1,3},(9.13)_{2}$ and (9.33), by letting $n \rightarrow \infty$ in (6.20), we deduce that the weak formulation of (6.15) is satisfied, i.e., (4.4) holds with $\left(\mathbf{u}, \mathbb{T}, \mathbb{T}_{0}\right)$ replaced by $\left(\mathbf{u}_{\sigma_{2}}, \mathbb{T}_{\sigma_{2}}, \mathbb{T}_{0, \theta}\right)$. In addition, from Lemmas 5.4 and $5.5,(9.18),(9.22)$ and $(9.12)_{3}$ we obtain that

$$
\varrho_{\sigma_{2}} \mathbf{u}_{\sigma_{2}} \in C_{w}\left([0, T] ; L^{\frac{2 \Gamma}{\Gamma+1}}\left(\Omega ; \mathbb{R}^{d}\right)\right) \quad \text { and } \quad \mathbb{T}_{\sigma_{2}} \in C_{w}\left([0, T] ; L^{2}\left(\Omega ; \mathbb{R}^{d \times d}\right)\right) .
$$

By the convergence results $(9.12),(9.13),(9.17),(9.43)_{1,2}$, (9.33), weak lower-semicontinuity of the norm in $L^{2}$ spaces, (9.14), (9.32) and Fatou's lemma, letting $n \rightarrow \infty$ in the inequalities (8.58) gives, for a.e. $t \in(0, T]$, the following inequalities:

$$
\begin{aligned}
E_{\sigma_{2}}(t)+4 \sigma_{2} \int_{0}^{t} & \int_{\Omega}\left(\frac{a}{\gamma}\left|\nabla_{x} \varrho_{\sigma_{2}}^{\frac{\gamma}{2}}\right|^{2}+\frac{\sigma_{1}}{\Gamma}\left|\nabla_{x} \varrho_{\sigma_{2}}^{\frac{\Gamma}{2}}\right|^{2}\right) \mathrm{d} x \mathrm{~d} t^{\prime}+\frac{1}{4 \lambda} \int_{0}^{t} \int_{\Omega} \operatorname{tr}\left(\left(\mathbb{A}\left(\mathbb{T}_{\sigma_{2}}\right)\right)^{2} \mathbb{T}_{\sigma_{2}}^{-1}\right) \mathrm{d} x \mathrm{~d} t^{\prime} \\
& +\int_{0}^{t} \int_{\Omega} \mu^{S}\left|\frac{\nabla_{x} \mathbf{u}_{\sigma_{2}}+\nabla_{x}^{\mathrm{T}} \mathbf{u}_{\sigma_{2}}}{2}-\frac{1}{d}\left(\operatorname{div}_{x} \mathbf{u}_{\sigma_{2}}\right) \mathbb{I}\right|^{2}+\mu^{B}\left|\operatorname{div}_{x} \mathbf{u}_{\sigma_{2}}\right|^{2} \mathrm{~d} x \mathrm{~d} t^{\prime} \\
& +\frac{\varepsilon}{2} \int_{0}^{t} \int_{\Omega}\left[\mathfrak{b}\left|\nabla_{x} \log \left(1-\frac{\operatorname{tr}\left(\mathbb{T}_{\sigma_{2}}\right)}{\mathfrak{b}}\right)\right|^{2}+\frac{\mathfrak{a}}{d}\left|\nabla_{x} \operatorname{tr}\left(\log \mathbb{T}_{\sigma_{2}}\right)\right|^{2}\right] \mathrm{d} x \mathrm{~d} t^{\prime} \\
\leq E_{0, \sigma_{1}, \theta} & +\int_{0}^{t} \int_{\Omega} \varrho_{\sigma_{2}} \mathbf{f} \cdot \mathbf{u}_{\sigma_{2}} \mathrm{~d} x \mathrm{~d} t^{\prime} \leq \mathrm{e}^{C t} E_{0, \sigma_{1}, \theta},
\end{aligned}
$$

where $C$ is a positive constant, independent of $t$ and of the approximation parameters $\left(\Gamma, \sigma_{1}, \sigma_{2}, \theta\right)$. Here the positive energy $E_{\sigma_{2}}$ is defined as

$$
E_{\sigma_{2}}(t):=\int_{\Omega}\left[\frac{1}{2} \varrho_{\sigma_{2}}\left|\mathbf{u}_{\sigma_{2}}\right|^{2}+\frac{a}{\gamma-1} \varrho_{\sigma_{2}}^{\gamma}+\frac{\sigma_{1}}{\Gamma-1} \varrho_{\sigma_{2}}^{\Gamma}-\Pi\left(\mathbb{T}_{\sigma_{2}}\right)+\frac{1}{2}(d \mathfrak{a} \log \mathfrak{a}+\mathfrak{b})\right] \mathrm{d} x,
$$

and the initial positive energy $E_{0, \sigma_{1}, \theta}$ is the same as in (7.20) and hence it is bounded by (7.23).

\section{THE FIRST LEVEL OF APPROXIMATION}

Now we let $\sigma_{2} \rightarrow 0$ in the solution sequence $\left(\varrho_{\sigma_{2}}, \mathbf{u}_{\sigma_{2}}, \mathbb{T}_{\sigma_{2}}\right)$, in order to deduce the existence of a solution to the first level of approximation, formulated in Section 6.2. First, we derive uniform bounds on $\left(\varrho_{\sigma_{2}}, \mathbf{u}_{\sigma_{2}}, \mathbb{T}_{\sigma_{2}}\right)$ as $\sigma_{2} \rightarrow 0$. Similarly to (9.1), it follows directly from (9.46) that

$$
\begin{aligned}
&\left\|\varrho_{\sigma_{2}}\right\|_{L^{\infty}\left(0, T ; L^{\gamma}(\Omega)\right)}+\sigma_{1}^{\frac{1}{\Gamma}}\left\|\varrho_{\sigma_{2}}\right\|_{L^{\infty}\left(0, T ; L^{\Gamma}(\Omega)\right)} \leq C\left(E_{0, \sigma_{1}, \theta}, T\right), \\
& \sqrt{\sigma_{2}}\left\|\nabla_{x}\left(\varrho_{\sigma_{2}}^{\frac{\gamma}{2}}\right)\right\|_{L^{2}\left((0, T) \times \Omega ; \mathbb{R}^{d}\right)}+\sqrt{\sigma_{1}} \sqrt{\sigma_{2}}\left\|\nabla_{x}\left(\varrho_{\sigma_{2}}^{\frac{\Gamma}{2}}\right)\right\|_{L^{2}\left((0, T) \times \Omega ; \mathbb{R}^{d}\right)} \leq C\left(E_{0, \sigma_{1}, \theta}, T\right), \\
&\left\|\varrho_{\sigma_{2}}\left|\mathbf{u}_{\sigma_{2}}\right|^{2}\right\|_{L^{\infty}\left(0, T ; L^{1}(\Omega)\right)}+\left\|\mathbf{u}_{\sigma_{2}}\right\|_{L^{2}\left(0, T ; W_{0}^{1,2}\left(\Omega ; \mathbb{R}^{d}\right)\right)} \leq C\left(E_{0, \sigma_{1}, \theta}, T\right), \\
&\left\|\Pi\left(\mathbb{T}_{\sigma_{2}}\right)\right\|_{L^{\infty}\left(0, T ; L^{1}(\Omega)\right)}+\left\|\operatorname{tr}\left(\left(\mathbb{A}\left(\mathbb{T}_{\sigma_{2}}\right)\right)^{2} \mathbb{T}_{\sigma_{2}}^{-1}\right)\right\|_{L^{1}\left(0, T ; L^{1}(\Omega)\right)} \leq C\left(E_{0, \sigma_{1}, \theta}, T\right), \\
&\left\|\nabla_{x} \operatorname{tr}\left(\log \mathbb{T}_{\sigma_{2}}\right)\right\|_{L^{2}\left(0, T ; L^{2}\left(\Omega ; \mathbb{R}^{d}\right)\right)}+\left\|\nabla_{x} \log \left(1-\frac{\operatorname{tr}\left(\mathbb{T}_{\sigma_{2}}\right)}{\mathfrak{b}}\right)\right\|_{L^{2}\left(0, T ; L^{2}\left(\Omega ; \mathbb{R}^{d}\right)\right)} \leq C\left(E_{0, \sigma_{1}, \theta}, T\right) .
\end{aligned}
$$

Next, we have the analogues of (9.3) and (9.5):

$$
\sqrt{\sigma_{2}}\left\|\nabla_{x} \varrho_{\sigma_{2}}\right\|_{L^{2}\left(0, T ; L^{2}\left(\Omega ; \mathbb{R}^{d}\right)\right)}+\left\|\partial_{t} \varrho_{\sigma_{2}}\right\|_{L^{2}\left(0, T ; W^{-1,2}(\Omega)\right)} \leq C\left(\sigma_{1}, E_{0, \sigma_{1}, \theta}, T\right) .
$$

In addition, we have the analogue of $(9.15)$ :

$(10.3)$

$$
\left\|\varrho_{\sigma_{2}} \mathbf{u}_{\sigma_{2}}\right\|_{L^{\infty}\left(0, T ; L^{\frac{2 \Gamma}{\Gamma+1}}\left(\Omega ; \mathbb{R}^{d}\right)\right)}+\left\|\varrho_{\sigma_{2}} \mathbf{u}_{\sigma_{2}}\right\|_{L^{2}\left(0, T ; L^{\frac{6 \Gamma}{\Gamma+6}}\left(\Omega ; \mathbb{R}^{d}\right)\right)}+\left\|\varrho_{\sigma_{2}}\left|\mathbf{u}_{\sigma_{2}}\right|^{2}\right\|_{L^{2}\left(0, T ; L^{\frac{6 \Gamma}{4 \Gamma+3}}\left(\Omega ; \mathbb{R}^{d \times d}\right)\right)} \leq C\left(\sigma_{1}, E_{0, \sigma_{1}, \theta}, T\right) .
$$

Next, we deduce from a Poincaré inequality, (1.8), (10.1) $4_{4,5}$ and (9.32) the analogues of (9.6) and (9.7):

$$
\begin{aligned}
\left\|(I-f) \Pi\left(\mathbb{T}_{\sigma_{2}}\right)\right\|_{L^{2}\left(0, T ; L^{2}(\Omega)\right)} & \leq C\left\|\nabla_{x} \Pi\left(\mathbb{T}_{\sigma_{2}}\right)\right\|_{L^{2}\left(0, T ; L^{2}(\Omega)\right)} \leq C\left(E_{0, \sigma_{1}, \theta}, T\right), \\
\left\|\mathbb{A}\left(\mathbb{T}_{\sigma_{2}}\right)\right\|_{L^{2}\left(0, T ; L^{2}\left(\Omega, \mathbb{R}^{d \times d}\right)\right)} & \leq C\left(E_{0, \sigma_{1}, \theta}, T\right) .
\end{aligned}
$$

Furthermore, we deduce from $(10.1)_{3},(10.4)_{2}$ and (9.32) the analogues of (9.9) and (9.11):

$$
\left\|\mathbb{T}_{\sigma_{2}}\right\|_{L^{\infty}\left(0, T ; L^{\infty}\left(\Omega ; \mathbb{R}^{d \times d}\right)\right)}+\left\|\mathbb{T}_{\sigma_{2}}\right\|_{L^{2}\left(0, T ; W^{1,2}\left(\Omega ; \mathbb{R}^{d \times d}\right)\right)}+\left\|\mathbb{T}_{\sigma_{2}}\right\|_{W^{1,2}\left(0, T ; W^{-1,2}\left(\Omega ; \mathbb{R}^{d \times d}\right)\right)} \leq C\left(E_{0, \sigma_{1}, \theta}, T\right) .
$$

It follows from $(10.1)_{1,3},(10.2)$ and (10.5) that, as $\sigma_{2} \rightarrow 0$,

$$
\begin{aligned}
\mathbf{u}_{\sigma_{2}} \rightarrow \mathbf{u}_{\sigma_{1}} & \text { weakly in } L^{2}\left(0, T ; W_{0}^{1,2}\left(\Omega ; \mathbb{R}^{d}\right)\right), \\
\varrho_{\sigma_{2}} \rightarrow \varrho_{\sigma_{1}} & \text { weakly-* in } L^{\infty}\left(0, T ; L^{\Gamma}(\Omega)\right) \cap W^{1,2}\left(0, T ; W^{-1,2}(\Omega)\right), \\
\mathbb{T}_{\sigma_{2}} \rightarrow \mathbb{T}_{\sigma_{1}} & \text { weakly-* in } L^{\infty}\left(0, T ; L^{\infty}\left(\Omega ; \mathbb{R}^{d \times d}\right)\right) \cap L^{2}\left(0, T ; W^{1,2}\left(\Omega ; \mathbb{R}^{d \times d}\right)\right) \cap W^{1,2}\left(0, T ; W^{-1,2}\left(\Omega ; \mathbb{R}^{d \times d}\right)\right) .
\end{aligned}
$$


The results $(10.6)_{2,3}$ and Lemma 5.3 yield that, as $\sigma_{2} \rightarrow 0$,

$$
\begin{aligned}
& \varrho_{\sigma_{2}} \rightarrow \varrho_{\sigma_{1}} \quad \text { strongly in } L^{2}\left(0, T ; W^{-1,2}(\Omega)\right) \text {, } \\
& \mathbb{T}_{\sigma_{2}} \rightarrow \mathbb{T}_{\sigma_{1}} \quad \text { strongly in } L^{2}\left(0, T ; L^{q}\left(\Omega ; \mathbb{R}^{d \times d}\right)\right) \text {, }
\end{aligned}
$$

where $q \in[1, \infty)$ if $d=2$ and $q \in[1,6)$ if $d=3$. We deduce from (10.3), (10.6) $)_{1},(10.7)_{1}$ and (10.2) that, as $\sigma_{2} \rightarrow 0$,

$$
\begin{aligned}
\varrho_{\sigma_{2}} \mathbf{u}_{\sigma_{2}} & \rightarrow \varrho_{\sigma_{1}} \mathbf{u}_{\sigma_{1}} & & \text { weakly-* in } L^{\infty}\left(0, T ; L^{\frac{2 \Gamma}{\Gamma+1}}\left(\Omega ; \mathbb{R}^{d}\right)\right) \cap L^{2}\left(0, T ; L^{\frac{6 \Gamma}{\Gamma+6}}\left(\Omega ; \mathbb{R}^{d}\right)\right), \\
\sigma_{2} \nabla_{x} \varrho_{\sigma_{2}} & \rightarrow \mathbf{0} & & \text { strongly in } L^{2}\left(0, T ; L^{2}\left(\Omega ; \mathbb{R}^{d}\right)\right) .
\end{aligned}
$$

We note the first result in (10.8) can be proved using a similar approach as in the proof of (9.23).

Using (10.7) 1 and (10.8), we can pass to the limit $\sigma_{2} \rightarrow 0$ in (9.26) to deduce that $\varrho_{\sigma_{1}}$ and $\mathbf{u}_{\sigma_{1}}$ satisfy (6.10) and the boundary condition (1.9) in the sense that

$$
\int_{0}^{T}\left\langle\partial_{t} \varrho_{\sigma_{1}}, \eta\right\rangle_{W^{1,2}(\Omega)} \mathrm{d} t-\int_{0}^{T} \int_{\Omega}\left(\varrho_{\sigma_{1}} \mathbf{u}_{\sigma_{1}}\right) \cdot \nabla_{x} \eta \mathrm{d} x \mathrm{~d} t=0 \quad \forall \eta \in L^{2}\left(0, T ; W^{1,2}(\Omega)\right) .
$$

The initial datum $\varrho_{0, \theta},(6.1)$, is attained in the sense of $C_{w}\left([0, T] ; L^{\Gamma}(\Omega)\right)$ by noting $(10.6)_{2}$ and Lemmas 5.4 and 5.5.

As $(9.32)$ holds, it is a simple matter to adapt the proof of Lemma 9.1, by noting $(10.1)_{4},(10.4)_{2}$ and $(10.7)_{2}$, to obtain its analogue.

Lemma 10.1. We have that

$$
\operatorname{tr}\left(\mathbb{T}_{\sigma_{1}}\right)<\mathfrak{b} \quad \text { and } \quad \mathbb{T}_{\sigma_{1}}>0 \quad \text { a.e. in }(0, T] \times \Omega .
$$

In addition, we have the following convergence result for $\sigma_{2} \rightarrow 0$ :

$$
\mathbb{A}\left(\mathbb{T}_{\sigma_{2}}\right) \rightarrow \mathbb{A}\left(\mathbb{T}_{\sigma_{1}}\right) \quad \text { strongly in } L^{r}\left(0, T ; L^{r}\left(\Omega ; \mathbb{R}^{d \times d}\right)\right),
$$

for any $r \in[1,2)$.

Similarly, as $(10.1)_{4},(10.4)_{1},(10.7)_{2}$ and (10.10) hold, it is a simple matter to adapt the proof of Lemma 9.2 to obtain its analogue.

Lemma 10.2. We have the following convergence results as $\sigma_{2} \rightarrow 0$ :

$$
\begin{aligned}
\nabla_{x} \operatorname{tr}\left(\log \mathbb{T}_{\sigma_{2}}\right) & \rightarrow \nabla_{x} \operatorname{tr}\left(\log \mathbb{T}_{\sigma_{1}}\right) & & \text { weakly in } L^{2}\left(0, T ; L^{2}(\Omega)\right), \\
\nabla_{x} \log \left(1-\frac{\operatorname{tr}\left(\mathbb{T}_{\sigma_{2}}\right)}{\mathfrak{b}}\right) & \rightarrow \nabla_{x} \log \left(1-\frac{\operatorname{tr}\left(\mathbb{T}_{\sigma_{1}}\right)}{\mathfrak{b}}\right) & & \text { weakly in } L^{2}\left(0, T ; L^{2}(\Omega)\right), \\
(I-f) \Pi\left(\mathbb{T}_{\sigma_{2}}\right) & \rightarrow(I-f) \Pi\left(\mathbb{T}_{\sigma_{1}}\right) & & \text { strongly in } L^{r}\left(0, T ; L^{r}(\Omega)\right),
\end{aligned}
$$

for any $r \in[1,2)$.

By noting (10.6) $)_{1,3},(10.7)$ and (10.11), we can pass to the limit $\sigma_{2} \rightarrow 0$ in the weak form of (6.15), i.e., (4.4) with $\left(\mathbf{u}, \mathbb{T}, \mathbb{T}_{0}\right)$ replaced by $\left(\mathbf{u}_{\sigma_{2}}, \mathbb{T}_{\sigma_{2}}, \mathbb{T}_{0, \theta}\right)$, to obtain the weak form of (6.12), i.e., (4.4) with $\left(\mathbf{u}, \mathbb{T}, \mathbb{T}_{0}\right)$ replaced by $\left(\mathbf{u}_{\sigma_{1}}, \mathbb{T}_{\sigma_{1}}, \mathbb{T}_{0, \theta}\right)$. Moreover, thanks to $(10.6)_{3}$ and Lemmas 5.4 and 5.5, we obtain that

$$
\mathbb{T}_{\sigma_{1}} \in C_{w}\left([0, T] ; L^{2}\left(\Omega ; \mathbb{R}^{d \times d}\right)\right) .
$$

The process of letting $\sigma_{2} \rightarrow 0$ in (9.44) to obtain (4.3) with the sextuple $\left(\varrho, \mathbf{u}, \mathbb{T}, p(\varrho), \varrho_{0}, \mathbf{u}_{0}\right)$ replaced by $\left(\varrho_{\sigma_{1}}, \mathbf{u}_{\sigma_{1}}, \mathbb{T}_{\sigma_{1}}, a \varrho_{\sigma_{1}}^{\gamma}+\sigma_{1} \varrho_{\sigma_{1}}^{\Gamma}, \varrho_{0, \theta}, \mathbf{u}_{0, \theta}\right)$ can be performed similarly as in the study of the compressible Navier-Stokes system; see for example Section 7.9 in [22], where the Bogovskil operator (cf. Lemma 5.2) is needed to show the higher integrability of the density, see Section 7.8.4 in [22], in order to prove convergence of the modified pressure. As the bounds (10.4) on the extra terms on the right-hand side of (9.44) due to the extra stress tensor, $\mathbb{A}\left(\mathbb{T}_{\sigma_{2}}\right)$, and the polymeric pressure, $(I-f) \Pi\left(\mathbb{T}_{\sigma_{2}}\right)$, are stronger than those, see (5.2) in [4], on the right-hand side of the corresponding approximation of the momentum equation for the compressible FENE model, see (4.118b) in [4], it is a simple matter to check that they do not affect the use of the Bogovskil operator to show that

$$
\left\|\varrho_{\sigma_{2}}\right\|_{\left.L^{\Gamma+1}(0, T) ; L^{\Gamma+1}(\Omega)\right)} \leq C\left(\sigma_{1}, E_{0, \sigma_{1}, \theta}, T\right) ;
$$

see Lemma 5.3 in [4]. In addition, the bounds (10.3) and Lemmas 5.4 and 5.5 are used to pass to the limit in the nonlinear terms $\varrho_{\sigma_{2}} \mathbf{u}_{\sigma_{2}}$ and $\varrho_{\sigma_{2}} \mathbf{u}_{\sigma_{2}} \otimes \mathbf{u}_{\sigma_{2}}$.

A key step in passing to the limit in a sequence of approximations to the compressible Navier-Stokes system is the proof of strong convergence of the approximations to the density, based on weak convergence of the, so called, effective viscous flux. Here, we recall Lemma 5.8, which is an extension of Lemma 7.36 in [22], suitable for dealing with the extra stress tensor, $\mathbb{A}\left(\mathbb{T}_{\sigma_{2}}\right)$, and the polymeric pressure, $(I-f) \Pi\left(\mathbb{T}_{\sigma_{2}}\right)$, on the right-hand side of (9.44). We note that we require here the extension, Lemma 5.8, as we only have the strong convergence results (10.11) and (10.12) $)_{3}$ for $\mathbb{A}\left(\mathbb{T}_{\sigma_{2}}\right)$ and $(I-f) \Pi\left(\mathbb{T}_{\sigma_{2}}\right)$, respectively, and not weak convergence results for their divergences. This is unlike the compressible 
Oldroyd-B model considered in [3], where this extension is not needed: Lemma 7.36 from [22] (suitably adapted to the case of $d=2$; cf. Lemma 5.8 in this paper with $\mathbb{B}_{n}=\mathbb{B}=\mathbb{O}$ ) directly applies, as, by recalling $(2.28), \mathbb{A}(\mathbb{T}) \equiv \mathbb{T}-\mathfrak{a} \mathbb{I}$ and so $(10.6)_{3}$ ensures fulfillment of the condition $(5.7)_{8}$ for the divergence of the extra stress tensor. We also recall from the paragraph below (2.28) that the polymeric pressure for the Oldroyd-B model in [3] is independent of $\mathbb{T}$ and depends only on $\eta,(2.8)$. In [3] we derived bounds on $\eta$, similar to those of $\mathbb{T}$, and so these ensure fulfillment of the condition $(5.7)_{8}$ for the divergence of the polymeric pressure.

Once again, it is a simple matter to check, as the bounds (10.4) in the present paper are stronger than (5.2) in [4], that the convergence argument in Section 5 in [4] for the corresponding approximation of the momentum equation for the compressible FENE model is easily adapted to (9.44). Hence, overall the limit $\left(\varrho_{\sigma_{1}}, \mathbf{u}_{\sigma_{1}}, \mathbb{T}_{\sigma_{1}}\right)$ is a solution to the weak formulation of (6.10)-(6.12), in the sense of $(10.9),(4.3)$ with $\left(\varrho, \mathbf{u}, \mathbb{T}, p(\varrho), \varrho_{0}, \mathbf{u}_{0}\right)$ replaced by $\left(\varrho_{\sigma_{1}}, \mathbf{u}_{\sigma_{1}}, \mathbb{T}_{\sigma_{1}}, a \varrho_{\sigma_{1}}^{\gamma}+\sigma_{1} \varrho_{\sigma_{1}}^{\Gamma}, \varrho_{0, \theta}, \mathbf{u}_{0, \theta}\right)$ and (4.4) with $\left(\mathbf{u}, \mathbb{T}, \mathbb{T}_{0}\right)$ replaced by $\left(\mathbf{u}_{\sigma_{1}}, \mathbb{T}_{\sigma_{1}}, \mathbb{T}_{0, \theta}\right)$. Of course, the nonnegativity of $\varrho_{\sigma_{1}}$ a.e. in $(0, T] \times \Omega$ follows from (9.14).

Similarly to the techniques used in obtaining the inequalities (9.46) from the inequalities (8.58), we can pass to the limit $\sigma_{2} \rightarrow 0$ in (9.46) to obtain the following inequalities, for a.e. $t \in(0, T]$ :

$$
\begin{aligned}
E_{\sigma_{1}}(t)+\frac{1}{4 \lambda} \int_{0}^{t} & \int_{\Omega} \operatorname{tr}\left(\left(\mathbb{A}\left(\mathbb{T}_{\sigma_{1}}\right)\right)^{2} \mathbb{T}_{\sigma_{1}}^{-1}\right) \mathrm{d} x \mathrm{~d} t^{\prime} \\
& +\int_{0}^{t} \int_{\Omega} \mu^{S}\left|\frac{\nabla_{x} \mathbf{u}_{\sigma_{1}}+\nabla_{x}^{\mathrm{T}} \mathbf{u}_{\sigma_{1}}}{2}-\frac{1}{d}\left(\operatorname{div}_{x} \mathbf{u}_{\sigma_{1}}\right) \mathbb{I}\right|^{2}+\mu^{B}\left|\operatorname{div}_{x} \mathbf{u}_{\sigma_{1}}\right|^{2} \mathrm{~d} x \mathrm{~d} t^{\prime} \\
& +\frac{\varepsilon}{2} \int_{0}^{t} \int_{\Omega}\left[\mathfrak{b}\left|\nabla_{x} \log \left(1-\frac{\operatorname{tr}\left(\mathbb{T}_{\sigma_{1}}\right)}{\mathfrak{b}}\right)\right|^{2}+\frac{\mathfrak{a}}{d}\left|\nabla_{x} \operatorname{tr}\left(\log \mathbb{T}_{\sigma_{1}}\right)\right|^{2}\right] \mathrm{d} x \mathrm{~d} t^{\prime} \\
\leq E_{0, \sigma_{1}, \theta} & +\int_{0}^{t} \int_{\Omega} \varrho_{\sigma_{1}} \mathbf{f} \cdot \mathbf{u}_{\sigma_{1}} \mathrm{~d} x \mathrm{~d} t^{\prime} \leq \mathrm{e}^{C t} E_{0, \sigma_{1}, \theta},
\end{aligned}
$$

where $C$ is a positive constant, independent of $t$ and of the approximation parameters $\left(\Gamma, \sigma_{1}, \theta\right)$. Here the positive energy $E_{\sigma_{1}}$ is defined as

$$
E_{\sigma_{1}}(t):=\int_{\Omega}\left[\frac{1}{2} \varrho_{\sigma_{1}}\left|\mathbf{u}_{\sigma_{1}}\right|^{2}+\frac{a}{\gamma-1} \varrho_{\sigma_{1}}^{\gamma}+\frac{\sigma_{1}}{\Gamma-1} \varrho_{\sigma_{1}}^{\Gamma}-\Pi\left(\mathbb{T}_{\sigma_{1}}\right)+\frac{1}{2}(d \mathfrak{a} \log \mathfrak{a}+\mathfrak{b})\right] \mathrm{d} x,
$$

and the initial positive energy $E_{0, \sigma_{1}, \theta}$ is the same as in (7.20) and hence is bounded by (7.23).

\section{Completion of the Proof}

11.1. Passage to the limits $\sigma_{1} \rightarrow 0$ and $\theta \rightarrow 0$. We shall simultaneously pass to the limits $\sigma_{1} \rightarrow 0$ and $\theta \rightarrow 0$. We need to show that the limit $(\varrho, \mathbf{u}, \mathbb{T})$ of the sequence $\left(\varrho_{\sigma_{1}}, \mathbf{u}_{\sigma_{1}}, \mathbb{T}_{\sigma_{1}}\right)$, as $\sigma_{1}, \theta \rightarrow 0$, is a weak solution to (1.1)-(1.10), (3.1) in the sense of Definition 4.1. We first show by choosing a suitable relationship between $\sigma_{1}$ and $\theta$ that $E_{0, \sigma_{1}, \theta} \rightarrow E_{0}$, recall (7.23) and (3.26), as $\sigma_{1}, \theta \rightarrow 0$.

Lemma 11.1. By choosing $\sigma_{1}=\theta^{s}$, where $s>2(\Gamma-\gamma)>0$ on recalling that $\Gamma>\max \{\gamma, 8\}$, we have that

$$
E_{0, \sigma_{1}, \theta} \rightarrow E_{0}, \quad \text { as } \sigma_{1} \rightarrow 0 .
$$

Proof. It follows from $(6.1)_{1,2}$ and (6.9) that the first two terms and the fourth term in $E_{0, \sigma_{1}, \theta},(7.23)$, converge to the first three terms in $E_{0},(3.26)$, as $\theta \rightarrow 0$. Therefore, it remains to show that the third term in $E_{0, \sigma_{1}, \theta}$ converges to 0 , as $\sigma_{1} \rightarrow 0$. For this, we have, by noting (6.2), (3.1) and (6.1), that

$$
\begin{aligned}
\sigma_{1} \int_{\Omega} \varrho_{0, \theta}^{\Gamma} \mathrm{d} x & =\sigma_{1}\left\|\varrho_{0, \theta}\right\|_{L^{\Gamma}(\Omega)}^{\Gamma} \leq \sigma_{1}\left\|\varrho_{0, \theta}\right\|_{L^{\gamma}(\Omega)}^{\gamma}\left\|\varrho_{0, \theta}\right\|_{L^{\infty}(\Omega)}^{\Gamma-\gamma} \\
& \leq C \sigma_{1}\left\|\theta+S_{\theta}\left[\varrho_{0}\right]\right\|_{L^{\gamma}(\Omega)}^{\gamma} \theta^{-2(\Gamma-\gamma)}\left\|\varrho_{0}\right\|_{L^{\gamma}(\Omega)}^{\gamma} \leq C \sigma_{1} \theta^{-2(\Gamma-\gamma)} .
\end{aligned}
$$

Hence, the desired result (11.1).

We now derive uniform bounds on $\left(\varrho_{\sigma_{1}}, \mathbf{u}_{\sigma_{1}}, \mathbb{T}_{\sigma_{1}}\right)$, as $\sigma_{1}=\theta^{s} \rightarrow 0$ with $s>2(\Gamma-\gamma)$. Similarly to (9.1), it follows directly from (10.15) and (11.1) that

$$
\begin{aligned}
& \left\|\varrho_{\sigma_{1}}\right\|_{L^{\infty}\left(0, T ; L^{\gamma}(\Omega)\right)}+\sigma_{1}^{\frac{1}{\Gamma}}\left\|\varrho_{\sigma_{1}}\right\|_{L^{\infty}\left(0, T ; L^{\Gamma}(\Omega)\right)} \leq C\left(E_{0}, T\right), \\
& \left\|\varrho_{\sigma_{1}}\left|\mathbf{u}_{\sigma_{1}}\right|^{2}\right\|_{L^{\infty}\left(0, T ; L^{1}(\Omega)\right)}+\left\|\mathbf{u}_{\sigma_{1}}\right\|_{L^{2}\left(0, T ; W_{0}^{1,2}\left(\Omega ; \mathbb{R}^{d}\right)\right)} \leq C\left(E_{0}, T\right),
\end{aligned}
$$

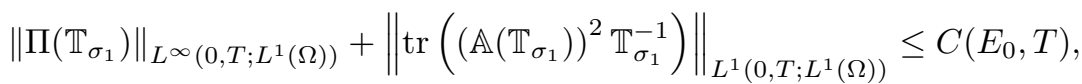

$$
\begin{aligned}
& \left\|\nabla_{x} \operatorname{tr}\left(\log \mathbb{T}_{\sigma_{1}}\right)\right\|_{L^{2}\left(0, T ; L^{2}\left(\Omega ; \mathbb{R}^{d}\right)\right)}+\left\|\nabla_{x} \log \left(1-\frac{\operatorname{tr}\left(\mathbb{T}_{\sigma_{1}}\right)}{\mathfrak{b}}\right)\right\|_{L^{2}\left(0, T ; L^{2}\left(\Omega ; \mathbb{R}^{d}\right)\right)} \leq C\left(E_{0}, T\right) .
\end{aligned}
$$


In addition, we have the analogues of (9.15) and (10.3):

$$
\left\|\varrho_{\sigma_{1}} \mathbf{u}_{\sigma_{1}}\right\|_{L^{\infty}\left(0, T ; L^{\frac{2 \gamma}{\gamma+1}}\left(\Omega ; \mathbb{R}^{d}\right)\right)}+\left\|\varrho_{\sigma_{1}} \mathbf{u}_{\sigma_{1}}\right\|_{L^{2}\left(0, T ; L^{\frac{6 \gamma}{\gamma+6}}\left(\Omega ; \mathbb{R}^{d}\right)\right)}+\left\|\varrho_{\sigma_{1}}\left|\mathbf{u}_{\sigma_{1}}\right|^{2}\right\|_{L^{2}\left(0, T ; L^{\frac{6 \gamma}{4 \gamma+3}}\left(\Omega ; \mathbb{R}^{d \times d}\right)\right)} \leq C\left(E_{0}, T\right) .
$$

We deduce from a Poincaré inequality, (1.8), (11.3) 3,4 and (10.10) the analogues of (9.6) and (9.7), as $\sigma_{1}=\theta^{s} \rightarrow 0$ with $s>2(\Gamma-\gamma)$ :

$$
\begin{aligned}
\left\|(I-f) \Pi\left(\mathbb{T}_{\sigma_{1}}\right)\right\|_{L^{2}\left(0, T ; L^{2}(\Omega)\right)} & \leq C\left\|\nabla_{x} \Pi\left(\mathbb{T}_{\sigma_{1}}\right)\right\|_{L^{2}\left(0, T ; L^{2}(\Omega)\right)} \leq C\left(E_{0}, T\right), \\
\left\|\mathbb{A}\left(\mathbb{T}_{\sigma_{1}}\right)\right\|_{L^{2}\left(0, T ; L^{2}\left(\Omega, \mathbb{R}^{d \times d}\right)\right)} & \leq C\left(E_{0}, T\right) .
\end{aligned}
$$

In addition, we deduce from $(11.3)_{2},(11.5)_{2}$ and (10.10) the analogues of (9.9) and (9.11), as $\sigma_{1}=\theta^{s} \rightarrow 0$ with $s>2(\Gamma-\gamma)$ :

$$
\left\|\mathbb{T}_{\sigma_{1}}\right\|_{L^{\infty}\left(0, T ; L^{\infty}\left(\Omega ; \mathbb{R}^{d \times d}\right)\right)}+\left\|\mathbb{T}_{\sigma_{1}}\right\|_{L^{2}\left(0, T ; W^{1,2}\left(\Omega ; \mathbb{R}^{d \times d}\right)\right)}+\left\|\mathbb{T}_{\sigma_{1}}\right\|_{W^{1,2}\left(0, T ; W^{-1,2}\left(\Omega ; \mathbb{R}^{d \times d}\right)\right)} \leq C\left(E_{0}, T\right) .
$$

It follows from $(11.3)_{2},(11.6)$ and Lemma 5.3 that, as $\sigma_{1}=\theta^{s} \rightarrow 0$ with $s>2(\Gamma-\gamma)$ :

$$
\begin{array}{ll}
\mathbf{u}_{\sigma_{1}} \rightarrow \mathbf{u} & \text { weakly in } L^{2}\left(0, T ; W_{0}^{1,2}\left(\Omega ; \mathbb{R}^{d}\right)\right), \\
\mathbb{T}_{\sigma_{1}} \rightarrow \mathbb{T} & \text { weakly-* in } L^{\infty}\left(0, T ; L^{\infty}\left(\Omega ; \mathbb{R}^{d \times d}\right)\right) \cap L^{2}\left(0, T ; W^{1,2}\left(\Omega ; \mathbb{R}^{d \times d}\right)\right) \cap W^{1,2}\left(0, T ; W^{-1,2}\left(\Omega ; \mathbb{R}^{d \times d}\right)\right), \\
\mathbb{T}_{\sigma_{1}} \rightarrow \mathbb{T} & \text { strongly in } L^{2}\left(0, T ; L^{q}\left(\Omega ; \mathbb{R}^{d \times d}\right)\right),
\end{array}
$$

where $q \in[1, \infty)$ if $d=2$ and $q \in[1,6)$ if $d=3$.

Furthermore, as (10.10) holds, it is a simple matter to adapt the proof of Lemma 9.1, by noting $(11.3)_{3},(11.5)_{2}$ and $(11.7)_{3}$, to obtain its analogue.

Lemma 11.2. We have that

$$
\operatorname{tr}(\mathbb{T})<\mathfrak{b} \quad \text { and } \quad \mathbb{T}>0 \quad \text { a.e. in }(0, T] \times \Omega .
$$

In addition, we have the following convergence result for $\sigma_{1}=\theta^{s} \rightarrow 0$ with $s>2(\Gamma-\gamma)$ :

$$
\mathbb{A}\left(\mathbb{T}_{\sigma_{1}}\right) \rightarrow \mathbb{A}(\mathbb{T}) \quad \text { strongly in } L^{r}\left(0, T ; L^{r}\left(\Omega ; \mathbb{R}^{d \times d}\right)\right),
$$

for any $r \in[1,2)$.

Similarly, as $(11.3)_{4},(11.5)_{1},(11.7)_{3}$ and (11.8) hold, it is a simple matter to adapt the proof of Lemma 9.2 to obtain its analogue.

Lemma 11.3. We have the following convergence results as $\sigma_{1}=\theta^{s} \rightarrow 0$ with $s>2(\Gamma-\gamma)$ :

$$
\begin{array}{rlrl}
\nabla_{x} \operatorname{tr}\left(\log \mathbb{T}_{\sigma_{1}}\right) & \rightarrow \nabla_{x} \operatorname{tr}(\log \mathbb{T}) & \text { weakly in } L^{2}\left(0, T ; L^{2}(\Omega)\right), \\
\nabla_{x} \log \left(1-\frac{\operatorname{tr}\left(\mathbb{T}_{\sigma_{1}}\right)}{\mathfrak{b}}\right) & \rightarrow \nabla_{x} \log \left(1-\frac{\operatorname{tr}(\mathbb{T})}{\mathfrak{b}}\right) & & \text { weakly in } L^{2}\left(0, T ; L^{2}(\Omega)\right), \\
(I-f) \Pi\left(\mathbb{T}_{\sigma_{1}}\right) & \rightarrow(I-f) \Pi(\mathbb{T}) & & \text { strongly in } L^{r}\left(0, T ; L^{r}(\Omega)\right),
\end{array}
$$

for any $r \in[1,2)$.

By noting (11.7) and (11.9), we can pass to the limit $\sigma_{1}=\theta^{s} \rightarrow 0$ with $s>2(\Gamma-\gamma)$ in the weak form of (6.12), i.e., (4.4) with the triple $(\mathbf{u}, \mathbb{T}, \mathbb{A}(\mathbb{T}))$ replaced by $\left(\mathbf{u}_{\sigma_{1}}, \mathbb{T}_{\sigma_{1}}, \mathbb{A}\left(\mathbb{T}_{\sigma_{1}}\right)\right)$, to obtain the weak form of (1.3), i.e., (4.4). Moreover, by $(11.7)_{2}$ and Lemmas 5.4 and 5.5 , we obtain that

$$
\mathbb{T} \in C_{w}\left([0, T] ; L^{2}\left(\Omega ; \mathbb{R}^{d \times d}\right)\right) .
$$

The process of letting $\sigma_{1} \rightarrow 0$ in (10.9) and (4.3) with the sextuple of functions $\left(\varrho, \mathbf{u}, \mathbb{T}, p(\varrho), \varrho_{0}, \mathbf{u}_{0}\right)$ replaced by $\left(\varrho_{\sigma_{1}}, \mathbf{u}_{\sigma_{1}}, \mathbb{T}_{\sigma_{1}}, a \varrho_{\sigma_{1}}^{\gamma}+\sigma_{1} \varrho_{\sigma_{1}}^{\Gamma}, \varrho_{0, \theta}, \mathbf{u}_{0, \theta}\right)$ to obtain (4.2) and (4.3), respectively, can be performed similarly as in the study of the compressible Navier-Stokes system; see for example Section 7.10 in [22], where once again the Bogovskiu operator (cf. Lemma 5.2) is needed to show the higher integrability of the density. It is a simple matter to check that the extra stress tensor, $\mathbb{A}\left(\mathbb{T}_{\sigma_{1}}\right)$, and the polymeric pressure, $(I-f) \Pi\left(\mathbb{T}_{\sigma_{1}}\right)$, with their strong bounds (11.5) compared to the bounds (6.2) in [4] $(d=3)$ and (3.11c) in [5] $(d=2)$ for the corresponding approximation of the momentum equation for the compressible FENE model, do not affect the use of the Bogovskiu operator; see Section 6 in [4] and Section 3 in [5] for $d=3$ and 2, respectively. In addition, the bounds (11.4) and Lemmas 5.4 and 5.5 are used to pass to the limit in the nonlinear terms $\varrho_{\sigma_{1}} \mathbf{u}_{\sigma_{1}}$ and $\varrho_{\sigma_{1}} \mathbf{u}_{\sigma_{1}} \otimes \mathbf{u}_{\sigma_{1}}$. Once again the proof of strong convergence of $\varrho_{\sigma_{1}}$ to $\varrho$ is based on weak convergence of the effective viscous flux using Lemma 5.8 and Corollary 5.9; see Section 6 in [4] and Section 3 in [5] for $d=3$ and 2, respectively. Finally, the nonnegativity of $\varrho$ a.e. in $(0, T] \times \Omega$ follows from the nonnegativity of $\varrho_{\sigma_{1}}$ a.e. in $(0, T] \times \Omega$. Thus, by observing the strong convergence of the initial data in (6.1) and (6.7), we deduce that the limit $(\varrho, \mathbf{u}, \mathbb{T})$ is a weak solution to $(1.1)-(1.10),(3.1)$ in the sense of Definition 4.1. The inequalities (4.8) follow by letting $\sigma_{1}=\theta^{s} \rightarrow 0$ with $s>2(\Gamma-\gamma)$ in (10.15). One can then deduce from (4.8) the bounds $(4.1)_{4,5}$ via the analogue of (11.5). The proof of Theorem 4.4 is thus complete. 


\section{REFERENCES}

[1] J. W. Barrett And S. Boyaval, Existence and approximation of a (regularized) Oldroyd-B model, Math. Models Methods Appl. Sci., 21 (2011), pp. 1783-1837.

[2] — Finite element approximation of the FENE-P model, IMA J. Numer. Anal. (to appear), (2018). Available from: https://academic.oup.com/imajna/advance-article/doi/10.1093/imanum/drx061/4561632.

[3] J. W. Barrett, Y. Lu, And E. Süli, Existence of large-data finite-energy global weak solutions to a compressible Oldroyd-B model, Commun. Math. Sci., 15 (2017), pp. 1265-1323.

[4] J. W. BARRetT AND E. SüLI, Existence of global weak solutions to compressible isentropic finitely extensible nonlinear bead-spring chain models for dilute polymers, Math. Models Methods Appl. Sci. 26 (2016), pp. 469-568.

[5] — Existence of global weak solutions to compressible isentropic finitely extensible nonlinear bead-spring chain models for dilute polymers: the two-dimensional case, J. Differential Equations, 261 (2016), pp. 592-626.

[6] R. Bird, P. Dotson, And N. Johnson, Polymer solution rheology based on a finitely extensible bead-spring chain model, J. NonNewtonian Fluid Mech., 7 (1980), pp. 213-235.

[7] M. E. Bogovskil, Solutions of some problems of vector analysis, associated with the operators div and grad, in Theory of cubature formulas and the application of functional analysis to problems of mathematical physics, vol. 1980 of Trudy Sem. S. L. Soboleva, No. 1, Akad. Nauk SSSR Sibirsk. Otdel., Inst. Mat., Novosibirsk, 1980, pp. 5-40, 149.

[8] S. Dain, Generalised Korn's inequality and conformal Killing vectors, Calc. Var. Partial Differential Equations, 25 (2006), pp. 535-540.

[9] L. C. Evans, Partial Differential Equations, vol. 19 of Graduate Studies in Mathematics, American Mathematical Society, Providence, RI, second ed., 2010.

[10] E. FeIREISL, On compactness of solutions to the compressible isentropic Navier-Stokes equations when the density is not square integrable, Comment. Math. Univ. Carolin., 42 (2001), pp. 83-98.

[11] — Dynamics of Viscous Compressible Fluids, vol. 26 of Oxford Lecture Series in Mathematics and its Applications, Oxford University Press, Oxford, 2004.

[12] E. Feireisl And A. Novotný, Singular Limits in Thermodynamics of Viscous Fluids, Advances in Mathematical Fluid Mechanics, Birkhauser, Basel, Boston, Berlin, 2009

[13] E. Feireisl, A. Novotný, And H. Petzeltová, On the existence of globally defined weak solutions to the Navier-Stokes equations, J. Math. Fluid Mech., 3 (2001), pp. 358-392.

[14] G. P. Galdi, An Introduction to the Mathematical Theory of the Navier-Stokes Equations. Steady-state Problems, Springer Monographs and Studies in Mathematics, Springer, New York, 2011.

[15] D. Gilbarg and N. S. Trudinger, Elliptic Partial Differential Equations of Second Order, Classics in Mathematics, Springer-Verlag, Berlin, 2001. Reprint of the 1998 edition.

[16] P. Grisvard, Elliptic Problems in Nonsmooth Domains, vol. 24 of Monographs and Studies in Mathematics, Pitman (Advanced Publishing Program), Boston, MA, 1985.

[17] D. Hu and T. Lelièvre, New entropy estimates for Oldroyd-B and related models, Commun. Math. Sci., 5 (2007), pp. $909-916$.

[18] R. Keunings, On the Peterlin approximation for finitely extensible dumbbells, J. Non-Newtonian Fluid Mech., 68 (1997), pp. 85-100.

[19] J.-L. Lions and E. Magenes, Non-Homogeneous Boundary Value Problems and Applications. Vol. I, Springer-Verlag, New York, 1972. Translated from the French by P. Kenneth, Die Grundlehren der mathematischen Wissenschaften, Band 181.

[20] P.-L. Lions, Mathematical Topics in Fluid Dynamics, Vol.2, Compressible models, Oxford Science Publication, Oxford, 1998.

[21] N. Masmoudi, Global existence of weak solutions to macroscopic models of polymeric flows, J. Math. Pures Appl., 96 (2011), pp. 502520 .

[22] A. Novotný And I. Straškraba, Introduction to the Mathematical Theory of Compressible Flow, Oxford University Press, Oxford, 2004.

[23] A. Peterlin, Hydrodynamics of macromolecules in a velocity field with longitudinal gradient, Polymer Letters, 4 (1966), pp. $287-291$.

[24] J. Simon, Compact sets in the space $L^{p}(0, T ; B)$, Ann. Math. Pura. Appl., 146 (1987), pp. 65-96.

[25] W. A. Strauss, On continuity of functions with values in various Banach spaces, Pacific J. Math., 19 (1966), pp. 543-551.

[26] P. Wapperom And M. Hulsen, Thermodynamics of viscoelastic fluids: The temperature equation, J. Non-Newtonian Fluid Mech., 42 (1998), pp. 999-1019.

[27] T. P. Winler, On the Hölder continuity of matrix functions for normal matrices, JIPAM. J. Inequal. Pure Appl. Math., 10 (2009), pp. Article 91, 5.

Department of Mathematics, Imperial College London, London SW7 2AZ, UK

E-mail address: j.barrett@imperial.ac.uk

Mathematical Institute, University of Oxford, Andrew Wiles Building, Woodstock Rd., Oxford OX2 6GG, UK

E-mail address: suli@maths.ox.ac.uk 